\title{
Observation and Measurement of Forward Proton Scattering in Association with Lepton Pairs Produced via the Photon Fusion Mechanism at ATLAS
}

\author{
G. Aad et al. \\ (ATLAS Collaboration)
}

(Received 2 October 2020; revised 30 October 2020; accepted 23 November 2020; published 23 December 2020)

\begin{abstract}
The observation of forward proton scattering in association with lepton pairs $\left(e^{+} e^{-}+p\right.$ or $\left.\mu^{+} \mu^{-}+p\right)$ produced via photon fusion is presented. The scattered proton is detected by the ATLAS Forward Proton spectrometer, while the leptons are reconstructed by the central ATLAS detector. Proton-proton collision data recorded in 2017 at a center-of-mass energy of $\sqrt{s}=13 \mathrm{TeV}$ are analyzed, corresponding to an integrated luminosity of $14.6 \mathrm{fb}^{-1}$. A total of $57(123)$ candidates in the $e e+p(\mu \mu+p)$ final state are selected, allowing the background-only hypothesis to be rejected with a significance exceeding 5 standard deviations in each channel. Proton-tagging techniques are introduced for cross-section measurements in the fiducial detector acceptance, corresponding to $\sigma_{e e+p}=11.0 \pm 2.6$ (stat) \pm 1.2 (syst) \pm 0.3 (lumi) and $\sigma_{\mu \mu+p}=7.2 \pm 1.6$ (stat) \pm 0.9 (syst) \pm 0.2 (lumi) fb in the dielectron and dimuon channel, respectively.

DOI: 10.1103/PhysRevLett.125.261801
\end{abstract}

Electromagnetic fields sourced by protons at the Large Hadron Collider (LHC) are sufficiently intense to exceed the Schwinger limit of $10^{18} \mathrm{~V} \mathrm{~m}^{-1}$ [1-3] and produce lepton pairs via photon fusion, $\gamma \gamma \rightarrow \ell^{+} \ell^{-}$, where $\ell$ denotes electrons or muons [4-7]. This process occurs in a wide range of astrophysical phenomena, such as cosmic gamma rays [8,9] and neutron stars [10,11]. Measurements of $\gamma \gamma \rightarrow \ell^{+} \ell^{-}$at the LHC provide a unique laboratory probe of these natural phenomena and are fundamental tests of quantum electrodynamics [12-17]. These complement lower-energy probes using heavy-ion collisions [18-26] and high-intensity laser beams [27-30]. A hallmark prediction of photon fusion processes at the LHC is the forward scattering of incident protons. Near-beam instruments known as proton spectrometers can detect the scattered protons, which is a technique referred to as proton tagging. The CMS and TOTEM Collaborations reported protontagged dielectron (dimuon) production with $2.6 \sigma(4.3 \sigma)$ significance, which exceeds $5 \sigma$ when statistically combined [31], but no cross sections were measured. Previous measurements of $\gamma \gamma \rightarrow \ell^{+} \ell^{-}$by the ATLAS Collaboration were performed without proton tagging $[4,5]$.

Measuring proton-tagged dilepton production, $p p \rightarrow$ $p\left(\gamma \gamma \rightarrow \ell^{+} \ell^{-}\right) p^{(*)}$, where $p^{(*)}$ denotes a proton that remains intact or dissociates following electromagnetic excitation, is important for several reasons. Predictions

*Full author list given at the end of the article.

Published by the American Physical Society under the terms of the Creative Commons Attribution 4.0 International license. Further distribution of this work must maintain attribution to the author(s) and the published article's title, journal citation, and DOI. Funded by SCOAP ${ }^{3}$. of photon fusion processes have significant uncertainties associated with modeling strong-force interactions between scattered protons, which suppress cross sections by factors known as soft-survival probabilities [32-35]. This suppression is poorly constrained, especially at high $\gamma \gamma$ invariant masses important for new physics searches, as existing probes indirectly infer dissociation rates using only centraldetector information [4-7]. Proton tagging overcomes this longstanding experimental ambiguity by directly detecting the scattered protons. Detecting a proton also directly suppresses background processes and events involving proton dissociation, while providing information on the initial $\gamma \gamma$ system independently of central-detector information. The successful demonstration of proton-tagging techniques for cross-section measurements accomplishes the crucial first step toward a diverse program using proton tagging in measurements of Standard Model processes [36-41] and searches for new phenomena [42-46].

This Letter introduces proton tagging for cross-section measurements of $p p \rightarrow p\left(\gamma \gamma \rightarrow \ell^{+} \ell^{-}\right) p^{(*)}$. The ATLAS Forward Proton (AFP) spectrometer detects one of the intact protons and the central ATLAS detector reconstructs the leptons. The dataset was collected in 2017 and corresponds to $14.6 \mathrm{fb}^{-1}$ of $\sqrt{s}=13 \mathrm{TeV}$ proton-proton $(p p)$ collisions. The average number of interactions per bunch crossing was 36 . Several methods specific to proton tagging are introduced: in situ calibration of proton kinematics using the dimuon system, a novel data-mixing background estimation method, and tag-and-probe determination of the AFP reconstruction efficiency.

The ATLAS experiment [47-49] is a general-purpose particle detector with nearly $4 \pi$ coverage [50] around the interaction point. It comprises an inner detector tracker, calorimeters, and a muon spectrometer. A two-level trigger 
system [51] is employed to select events containing sameflavor lepton pairs, each lepton with $p_{T}^{e(\mu)}>17(14) \mathrm{GeV}$ [52-54], after which standard data-quality requirements are applied [55].

The AFP spectrometer $[56,57]$ consists of four tracking units located along the beam pipe at $z= \pm 205$ and $\pm 217 \mathrm{~m}$, referred to as near and far stations, respectively. The $+z(-z)$ direction is labeled side $A(C)$. Each station houses a silicon tracker comprising four planes of edgeless silicon pixel sensors [58-61]. The sensors have $336 \times$ 80 pixels with area $50 \times 250 \mu \mathrm{m}^{2}$. The direction normal to each sensor is tilted $14^{\circ}$ relative to the beam to improve hit efficiency and $x$-position resolution, resulting in an overall spatial resolution of $\sigma_{x}=6 \mu \mathrm{m}$ [62]. Movable near-beam devices at each station, known as Roman pots, insert the tracker along the $x$ direction in the beam pipe. Data taking with the AFP commences once the trackers are at a position where the innermost silicon edge is within $2 \mathrm{~mm}$ of the beam center during stable beams. Data quality for this analysis requires that every AFP station has at least three silicon planes operational at high voltage, and the AFP data acquisition system [63] must report no problems.

Simulated events of the exclusive signal $p p \rightarrow$ $p\left(\gamma \gamma \rightarrow \ell^{+} \ell^{-}\right) p$ were produced using the HERWIG7 Monte Carlo (MC) generator $[64,65]$. The single-dissociative signal $p p \rightarrow p\left(\gamma \gamma \rightarrow \ell^{+} \ell^{-}\right) p^{*}$ was generated using LPAIR4.0 [66], with proton dissociation modeled using the Brasse et al. [67] and Suri-Yennie [68] structure functions interfaced with JETSET7.408 [69,70]. Simulation of these processes is detailed in Ref. [5]. To model the centraldetector response, the exclusive signal sample underwent full detector simulation based on GEANT4 [71]. The singledissociative samples employed a fast simulation [72], which uses a parametrization of the calorimeter response [73]. The response of the AFP spectrometer is modeled by a fast simulation, where a Gaussian smearing is applied to track positions based on the AFP spatial resolution. Simulated samples include the effect on the central detector of multiple $p p$ interactions in the same and neighboring bunch crossing (pileup), as detailed in Ref. [5].

Reconstructed events must contain at least one interaction vertex with two or more associated inner-detector tracks that satisfy $p_{T}>500 \mathrm{MeV},|\eta|<2.5$, and the "Loose" criterion [74,75]. Electrons (muons) must satisfy $p_{T}>$ 18(15) GeV, $|\eta|<2.47(2.4)$, the "LooseAndBLayer" [76] ("Medium" [77]) identification criterion, and $\left|z_{0} \sin \theta\right|<$ $0.5 \mathrm{~mm}$ [78]. Electrons sharing an inner-detector track with a muon are discarded. To suppress fake and/or nonprompt lepton backgrounds, remaining electrons (muons) must satisfy transverse impact parameter significance $\left|d_{0} / \sigma_{d_{0}}\right|<$ 5(3) and isolation requirements described in Ref. [79] (Ref. [80]). Electrons must also satisfy "Medium" identification [76]. Small corrections are applied to leptons in simulated samples to match reconstruction and trigger efficiencies measured in data, as described in Refs. [76,77].
Selected events must have exactly two same-flavor leptons with opposite electric charge $\left(e^{+} e^{-}\right.$or $\left.\mu^{+} \mu^{-}\right)$and be matched to the leptons that triggered the event. To suppress quarkonia and $Z$ boson resonances, the dilepton invariant mass must satisfy $m_{\ell \ell}>20$ and $m_{\ell \ell} \notin[70,105] \mathrm{GeV}$. To select events compatible with $p p \rightarrow p\left(\gamma \gamma \rightarrow \ell^{+} \ell^{-}\right) p^{(*)}$ processes based on the simulated signals, the dilepton transverse momentum must satisfy $p_{T}^{\ell \ell}<5 \mathrm{GeV}$. This set of criteria is referred to as the preselection. Signal event candidates must additionally have small acoplanarity $A_{\phi}^{\ell \ell}=1-\left|\Delta \phi_{\ell \ell}\right| / \pi<0.01$. These events must have no inner-detector tracks $\left(N_{\text {tracks }}^{0.5 \mathrm{~mm}}=0\right)$ that satisfy $\Delta R(\operatorname{track}, \ell)>0.01$ for both leptons and $\left|z_{0}^{\text {track }}-z_{0}^{\ell \ell}\right|<0.5 \mathrm{~mm}$, where $z_{0}^{\text {track }}$ is the track $z_{0}$ position and $z_{0}^{\ell \ell}=\left(z_{0}^{\ell_{1}}+z_{0}^{\ell_{2}}\right) / 2$ with $\ell_{1,2}$ denoting the two leptons. The expected proton energy loss based on lepton kinematics $\xi_{\ell \ell}$ is determined from $m_{\ell \ell}$ and the dilepton rapidity $y_{\ell \ell}$ by momentum conservation $\xi_{\ell \ell} \pm=\left(m_{\ell \ell} / \sqrt{s}\right) e^{ \pm y_{\ell \ell}}$, where + $(-)$ corresponds to the proton on side $A(C)$.

Reconstruction of scattered protons combines information from the AFP tracker and LHC magnet lattice [81]. Protons transported to the AFP leave hits in the silicon tracker, which are processed by clustering and track-finding algorithms detailed in Ref. [59]. Tracks are reconstructed from clusters in at least two planes. Small corrections of around $0.1 \mathrm{~mm}$ are applied to ensure the cluster positions between planes are compatible within the spatial resolution. The proton transport function $x_{\mathrm{AFP}}=T\left(\xi_{\mathrm{AFP}}\right)$ relates the track $x$ position $x_{\mathrm{AFP}}$ to the fractional energy loss of the scattered proton $\xi_{\mathrm{AFP}}=1-E_{\text {scattered }} / E_{\text {beam }}$, where $E_{\text {scattered }}\left(E_{\text {beam }}\right)$ is the scattered (beam) proton energy. The LHC magnets and beam optics [82] govern the form of $T\left(\xi_{\mathrm{AFP}}\right)$ [83], which is simulated in the MAD-X package $[84,85]$ with further details discussed in Refs. [56,86,87]. Determination of $\xi_{\text {AFP }}$ uses both the near and far stations if tracks are within their common acceptance, otherwise only the far station is used.

The absolute scale of $E_{\text {scattered }}$ depends on the closest separation $x_{0}^{s}$ between each AFP station $s$ and the beam center [87]. The beam positions relative to the detectors were determined in dedicated runs with beam-based alignment procedures [88] using beam loss monitors [89], and cross-checked with beam position monitor measurements [90]. There were three data-taking periods in 2017. In the first data-taking period, the $x_{0}^{s}$ values were initially set to $-4.0(-3.0) \mathrm{mm}$ on side $A$ and $-3.8(-2.9) \mathrm{mm}$ on side $C$ for the near (far) stations; during a second data-taking period, all stations were moved $0.5 \mathrm{~mm}$ closer to the beam to improve acceptance. This first (second) data-taking period corresponds to $5 \%(17 \%)$ of the analyzed dataset. For the remaining dataset, the far stations were moved a further $0.2 \mathrm{~mm}$ toward the beam. The initially measured $x_{\mathrm{AFP}}$ values relative to $x_{0}^{s}$ are calibrated in situ using the dimuon data sample passing the signal event selection. The $x_{\ell \ell}^{s}-x_{\mathrm{AFP}}^{s}$ distribution is peaked for signal processes due to the kinematic correlation between $x_{\ell \ell}^{s}$ and $x_{\mathrm{AFP}}^{s}$, where 
$x_{\ell \ell}=T\left(\xi_{\ell \ell}\right)$ is the expected position calculated using the transport function. Additive corrections are applied to $x_{\mathrm{AFP}}^{s}$ in data to center the maximum of the peak at zero. These corrections are found to be $-0.28(-0.34) \mathrm{mm}$ on side $A$ and $-0.17(-0.36) \mathrm{mm}$ on side $C$ for the near (far) stations. Selected dielectron events are used to verify that the signal is centered at zero. After applying these corrections, the lower value of the acceptance corresponds to $\xi_{\mathrm{AFP}}^{\mathrm{A}}>$ $0.028(0.018)$ on side $A$ and $\xi_{\mathrm{AFP}}^{\mathrm{C}}>0.026(0.019)$ on side $C$ for the near (far) stations. The upper value of the acceptance is bounded by $\xi_{\mathrm{AFP}}<0.12$ due to the presence of beam collimators [56].

To select events with one or more proton candidates, the $\xi_{\ell \ell}$ and $\xi_{\mathrm{AFP}}$ values for at least one AFP side are required to be within the range $[0.02,0.12]$. If there is more than one proton candidate on the same AFP side, which occurs in $35 \%$ of selected events, the proton with $\xi_{\text {AFP }}$ closest to $\xi_{l \ell}$ is chosen. Proton-tagged dilepton candidates, denoted $\ell \ell+p$, are selected by requiring kinematic matching on at least one AFP side, $\left|\xi_{\mathrm{AFP}}-\xi_{\ell \ell}\right|<0.005$, which retains (rejects) more than 95\% (85\%) of the signal (background).

The dominant source of background after this selection arises from lepton pairs produced in a $p p$ interaction different from that of the detected proton. In this case, the lepton pairs are produced via the Drell-Yan mechanism, as well as $\gamma \gamma \rightarrow \ell^{+} \ell^{-}$processes, in which any outgoing protons are either outside the AFP acceptance or not reconstructed in AFP due to detector inefficiency. These events are collectively referred to as combinatorial backgrounds and are estimated using a data-driven method. A mixed-data sample is constructed by randomly pairing each measured $\xi_{\ell \ell}$ value, passing AFP acceptance $\xi_{\mathrm{AFP}} \in[0.02,0.12]$, with 100 values of $\xi_{\mathrm{AFP}}$ from a large control sample of $>10^{6}$ events. This control sample is constructed from the preselected events and requiring $A_{\phi}^{\ell \ell}>0.01$. The 123 selected data events failing kinematic matching, $\left|\xi_{\mathrm{AFP}}-\xi_{\ell \ell}\right|>0.005$, result mostly from combinatorial background processes, which are used to normalize the mixed-data sample using a background-only profile-likelihood fit $[91,92]$.

Systematic uncertainties in the background normalization arise from the limited size of the data sample satisfying $\left|\xi_{\text {AFP }}-\xi_{\text {e }}\right|>0.005$. An uncertainty in the background shape arises from kinematic changes in the control sample of protons due to the acoplanarity requirement. This uncertainty is estimated by replacing the $A_{\phi}^{\ell \ell}>0.01$ condition with $N_{\text {tracks }}^{0.5 \mathrm{~mm}} \geq 1$ and comparing the two background predictions in the region $\left|\xi_{\text {AFP }}-\xi_{\text {ee }}\right|<0.005$; they are found to differ by $14 \%$. Further shape uncertainties arise from instrumental effects, which are expected to be dominated by the sensitivity to the number of interactions per bunch crossing $\mu$. The background predictions for $\mu<35$ and $\mu \geq 35$ are found to differ by $8 \%$ in the $\left|\xi_{\text {AFP }}-\xi_{\ell \ell}\right|<0.005$ region. These two shape differences are assigned as additional uncertainties.

The background estimation method is validated by applying it to the orthogonal $m_{\ell \ell} \in[70,105] \mathrm{GeV}$ region. The region $\left|\xi_{\mathrm{AFP}}-\xi_{\ell \ell}\right|>0.005$ is dominated by Drell-Yan events, which have no correlated protons. In this region, the data and prediction from the mixed-data sample are found to be compatible within the uncertainties across the $\xi_{\mathrm{AFP}}-$ $\xi_{\ell \ell}$ range for both sides $A$ and $C$.

After applying the event selection including kinematic matching, $\left|\xi_{\mathrm{AFP}}-\xi_{\ell \ell}\right|<0.005$, a total of 57 (123) candidates in the $e e+p(\mu \mu+p)$ final state are observed compared with a background-only expectation of $6.2 \pm$ $1.2(13.4 \pm 2.5)$ events. Using the asymptotic profilelikelihood method [91,92], the background-only hypothesis is rejected with a significance exceeding $5 \sigma$ in each channel [93]. This provides direct evidence of forward proton scattering in association with electron and muon pairs produced via photon fusion. The $\xi_{\mathrm{AFP}}-\xi_{l \ell}$ distributions of data, signal, and background at detector level before kinematic matching are shown in Fig. 1. To illustrate

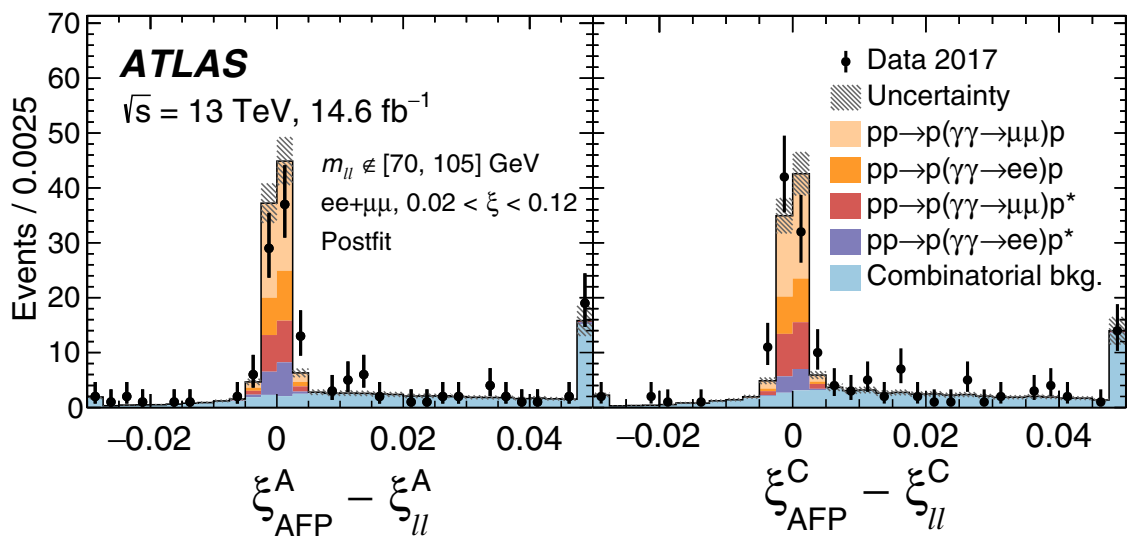

FIG. 1. Distributions of $\xi_{\mathrm{AFP}}-\xi_{\ell \ell}$ with $\xi_{\ell \ell}$ and $\xi_{\mathrm{AFP}}$ satisfying [0.02, 0.12] for side $A$ (left) and side $C$ (right). The total prediction comprises the signal and combinatorial background processes, where $p^{*}$ denotes a dissociated proton. The simulated predictions are normalized to data to illustrate the expected signal composition. The first (last) bin includes underflow (overflow). The hatched band indicates the combined statistical and systematic uncertainties of the prediction. Error bars denote statistical uncertainties of the data. 


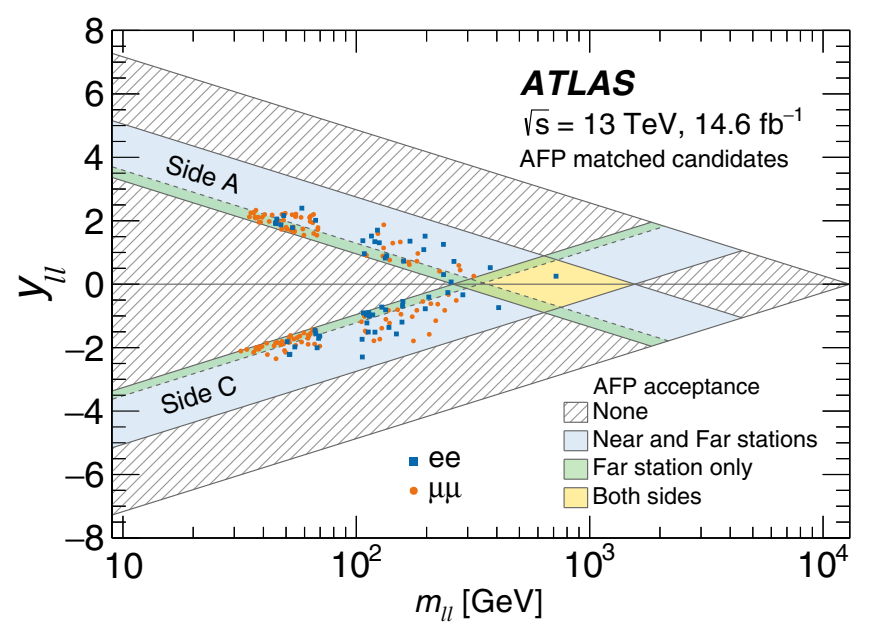

FIG. 2. The $57(123)$ ee $(\mu \mu)$ data event candidates in the dilepton rapidity $y_{\ell \ell}$ vs $m_{\ell \ell}$ plane satisfying event selection and kinematic matching, $\left|\xi_{\mathrm{AFP}}-\xi_{\ell \ell}\right|<0.005$, on at least one side. Shaded (hatched) areas denote the acceptance (no acceptance) for the AFP stations indicated in the legend. Areas neither shaded nor hatched correspond to $\xi \notin[0,1]$.

the expected composition of the signal, the simulated samples are normalized to data with sides $A$ and $C$ combined and fit separately in the $e e$ and $\mu \mu$ channels. Figure 2 displays positions in the $y_{\ell \ell}-m_{\ell \ell}$ plane of data candidates satisfying $\left|\xi_{\mathrm{AFP}}-\xi_{\ell \ell}\right|<0.005$ on at least one side and the corresponding acceptance regions of the four AFP stations. The highest-mass $e e$ candidate has an invariant mass $m_{\ell \ell}=717 \mathrm{GeV}$ and rapidity $y_{\ell \ell}=0.252$, so the scattered protons would be within the acceptance of both AFP sides if this were an exclusive process. However, it is found that the proton on side $A$ fails kinematic matching $\left|\xi_{\mathrm{AFP}}-\xi_{\ell \ell}\right|<0.005$, so this event is likely a single-dissociative process where the side $A$ proton candidate originates from a pileup interaction. The corresponding quantities for the highest-mass $\mu \mu$ candidate are $m_{\ell \ell}=319 \mathrm{GeV}$ and $y_{\ell \ell}=0.255$. Figure 3 illustrates detector-level distributions of dilepton acoplanarity, mass, and rapidity after kinematic matching with the signal samples normalized to $N_{\text {obs }}-N_{\text {bkg }}$.

Cross sections are measured in a fiducial region defined at particle level with an event selection similar to that applied at detector level [94]. To reliably estimate AFP reconstruction efficiencies using tag-and-probe techniques, the $\xi_{\mathrm{AFP}}$ and $\xi_{\ell \ell}$ values are restricted to a tighter range $[0.035,0.08]$ and each proton candidate is required to have an associated track in both near and far stations. The measured cross sections are defined by $\sigma_{\text {fid. }}=\left(N_{\text {obs }}-N_{\text {bkg }}\right) /\left(\mathcal{L} \cdot C_{\text {cent }} \cdot C_{\text {AFP }}\right)$. Here, $N_{\text {obs }}\left(N_{\text {bkg }}\right)$ is the number of observed data (expected background) events passing event selection, and $C_{\text {cent }}\left(C_{\mathrm{AFP}}\right)$ is an overall correction factor accounting for the centraldetector (AFP) efficiency. The integrated luminosity, $\mathcal{L}=14.6 \mathrm{fb}^{-1}$, is measured using the LUCID-2 detector [95] and the uncertainty is determined to be $2.4 \%$ [96]. In this tighter region, $N_{\text {obs }}$ is found to be 19 (23) for the ee $(\mu \mu)$ channel and $N_{\mathrm{bkg}}=1.7 \pm 0.3(2.3 \pm 0.5)$. The event rate between the two channels differs more for the $\xi \in$ $[0.02,0.12]$ than $\xi \in[0.035,0.08]$ region because $\mu \mu$ events with low $m_{\ell \ell}$ and high $\left|y_{\ell \ell}\right|$ have greater selection efficiency due to trigger and reconstruction requirements.

The $C_{\text {cent }}$ factor is defined as the ratio of the number of MC events passing detector-level selection to the number passing the particle-level fiducial requirements. Uncertainties in $C_{\text {cent }}$ are estimated by varying the electron (muon) energy (momentum) scale and resolution, and datato-MC correction factors described in Refs. [76,77], together with corrections applied to account for pileup modeling. The dominant uncertainties for $e e$ events arise from pileup modeling (2\%) and identification (1\%), while for $\mu \mu$ events, these correspond to pileup modeling (3\%), resolution (3\%), and scale (2\%); other sources such as trigger and isolation efficiencies contribute $1 \%$ or less. Using data-driven methods described in Ref. [5], a further correction of $0.89 \pm 0.04$ is applied to $C_{\text {cent }}$ to account for
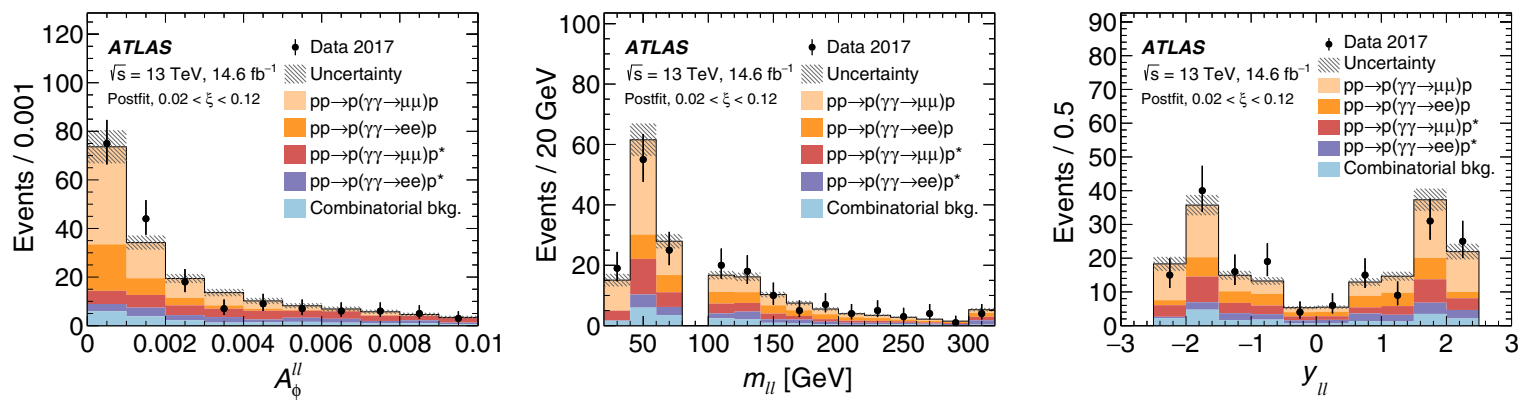

FIG. 3. Distributions of dilepton acoplanarity $A_{\phi}^{\ell \ell}$ (left), invariant mass $m_{\ell \ell}$ (center), rapidity $y_{\ell \ell}$ (right) satisfying $\xi_{\ell \ell}, \xi_{\mathrm{AFP}} \in[0.02,0.12]$, and $\left|\xi_{\mathrm{AFP}}-\xi_{\ell \ell}\right|<0.005$ for at least one AFP side. Events with $70<m_{\ell \ell}<105 \mathrm{GeV}$ are vetoed. The total prediction comprises the signal and combinatorial background processes, where $p^{*}$ denotes a dissociated proton. The simulated predictions are normalized to data to illustrate the expected signal composition. The rightmost bin of the $m_{\ell \ell}$ distribution includes overflow. The hatched band indicates the combined statistical and systematic uncertainties of the prediction. Error bars denote statistical uncertainties of the data. 
differences between data and $\mathrm{MC}$ when modeling the luminous region at the interaction point. The $5 \%$ uncertainty in this correction is evaluated as the difference between either applying this data-driven method to simulated signal samples or imposing the $N_{\text {tracks }}^{0.5 \mathrm{~mm}}=0$ requirement on these samples. Overall, this results in $C_{\text {cent }}^{e e}=0.12 \pm 0.01$ $\left(C_{\text {cent }}^{\mu \mu}=0.22 \pm 0.02\right)$ for the $e e(\mu \mu)$ channel.

The $C_{\mathrm{AFP}}$ factor is defined by the product $\epsilon_{\text {track }} \cdot \epsilon_{\text {smear }}$. The track reconstruction efficiency $\epsilon_{\text {track }}$ is found to be $0.92 \pm 0.02$ for sides $A$ and $C$. The near-station efficiency is estimated using a tag-and-probe method by first selecting events with exactly one track in the far (tag) station in the acceptance common to both stations, $-12<x_{\mathrm{AFP}}<-5 \mathrm{~mm}$. The efficiency is the fraction of these events that also have one or more tracks in the near (probe) station satisfying $\left|x_{\text {near }}-x_{\text {far }}\right|<2 \mathrm{~mm}$. The tag and probe stations are inverted to measure the far-station efficiency. It is found that $\epsilon_{\text {track }}$ varies with $\xi_{\text {AFP }}$ by $2 \%$, which is assigned as an additional uncertainty. The proton resolution correction $\epsilon_{\text {smear }}$ is found to be $0.98 \pm 0.02$ $(0.96 \pm 0.04)$ for the $e e(\mu \mu)$ channel. This is evaluated as the fraction of simulated signal events passing $\xi_{\mathrm{AFP}}, \xi_{\ell \ell} \in[0.035,0.08]$, and $\left|\xi_{\mathrm{AFP}}-\xi_{\ell \ell}\right|<0.005$ out of those satisfying $\xi_{\ell \ell} \in[0.035,0.08]$. Uncertainties in $C_{\mathrm{AFP}}$ are dominated by global alignment $(6 \%)$ evaluated by $\pm 0.3 \mathrm{~mm}$ variations of $x_{\mathrm{AFP}}$ and beam optics (5\%) evaluated by varying the beam crossing angle by $50 \mu \mathrm{rad}$ in the MAD-X package. Uncertainties involving track and cluster reconstruction are found to be less than $1 \%$. The overall uncertainty in $C_{\mathrm{AFP}}$ is $9 \%$.

The measured fiducial cross sections in the $e e$ and $\mu \mu$ channels are $\sigma_{e e+p}^{\text {fid. }}=11.0 \pm 2.6$ (stat) \pm 1.2 (syst) \pm 0.3 (lumi) and $\sigma_{\mu \mu+p}^{\text {fid. }}=7.2 \pm 1.6$ (stat) \pm 0.9 (syst) \pm 0.2 (lumi) fb, respectively. Table I compares these with the combined HERWIG and LPAIR predictions assuming unit soft-survival factors $S_{\text {surv }}=1$. Soft-survival effects are included using an $m_{\ell \ell}$-dependent reweighting of these predictions to $S_{\text {surv }}$ calculated for exclusive processes from Ref. [34]; LPAIR predictions are additionally scaled down by $15 \%$ to account for $S_{\text {surv }}$ being lower for single-dissociative processes [33].

TABLE I. Fiducial cross sections from the combined HERWIG and LPAIR predictions with $S_{\text {surv }}=1$ and $S_{\text {surv }}$ estimated using Refs. [33,34] as described in the main text. SUPERCHIC 4 [97] predictions include fully kinematically dependent $S_{\text {surv }}$. Uncertainties of 7\% (17\%) are assigned for predictions of the exclusive (single-dissociative) processes [98]. The bottom row displays the measured cross sections with statistical and systematic uncertainties combined.

\begin{tabular}{llr}
\hline \hline$\sigma_{\text {HERWIG }+ \text { LPAIR }} \times S_{\text {surv }}$ & $\sigma_{e e+p}^{\text {fid. }}(\mathrm{fb})$ & \multicolumn{1}{c}{$\sigma_{\mu \mu+p}^{\text {fid. }}(\mathrm{fb})$} \\
\hline$S_{\text {surv }}=1$ & $15.5 \pm 1.2$ & $13.5 \pm 1.1$ \\
$S_{\text {surv using Refs. [33,34] }}$ & $10.9 \pm 0.8$ & $9.4 \pm 0.7$ \\
SUPERCHIC 4 [97] & $12.2 \pm 0.9$ & $10.4 \pm 0.7$ \\
Measurement & $11.0 \pm 2.9$ & $7.2 \pm 1.8$ \\
\hline \hline
\end{tabular}

SUPERCHIC 4 [97] predictions include full kinematic dependence on $S_{\text {surv }}$ for exclusive, single-, and doubledissociative processes. The predictions for $e e$ are higher than for $\mu \mu$ due to the looser $\eta(e)$ requirement [94].

In summary, forward proton scattering in association with lepton pairs produced via photon fusion, $p p \rightarrow$ $p\left(\gamma \gamma \rightarrow \ell^{+} \ell^{-}\right) p^{(*)}$, is observed with a significance exceeding $5 \sigma$ in both the $e e+p$ and $\mu \mu+p$ final states using $14.6 \mathrm{fb}^{-1}$ of $\sqrt{s}=13 \mathrm{TeV} p p$ collisions at the LHC. These results demonstrate that the ATLAS Forward Proton spectrometer performs well in high-luminosity data taking. Furthermore, proton tagging is introduced for crosssection measurements of photon fusion processes at the electroweak scale.

We thank CERN for the very successful operation of the LHC, as well as the support staff from our institutions without whom ATLAS could not be operated efficiently. We acknowledge the support of ANPCyT, Argentina; YerPhI, Armenia; ARC, Australia; BMWFW and FWF, Austria; ANAS, Azerbaijan; SSTC, Belarus; CNPq and FAPESP, Brazil; NSERC, NRC and CFI, Canada; CERN; ANID, Chile; CAS, MOST and NSFC, China; COLCIENCIAS, Colombia; MSMT CR, MPO CR and VSC CR, Czech Republic; DNRF and DNSRC, Denmark; IN2P3-CNRS and CEA-DRF/IRFU, France; SRNSFG, Georgia; BMBF, HGF and MPG, Germany; GSRT, Greece; RGC and Hong Kong SAR, China; ISF and Benoziyo Center, Israel; INFN, Italy; MEXT and JSPS, Japan; CNRST, Morocco; NWO, Netherlands; RCN, Norway; MNiSW and NCN, Poland; FCT, Portugal; MNE/IFA, Romania; MES of Russia and NRC KI, Russia Federation; JINR; MESTD, Serbia; MSSR, Slovakia; ARRS and MIZŠ, Slovenia; DST/NRF, South Africa; MICINN, Spain; SRC and Wallenberg Foundation, Sweden; SERI, SNSF and Cantons of Bern and Geneva, Switzerland; MOST, Taiwan; TAEK, Turkey; STFC, United Kingdom; DOE and NSF, U.S. In addition, individual groups and members have received support from BCKDF, CANARIE, Compute Canada, CRC and IVADO, Canada; Beijing Municipal Science \& Technology Commission, China; COST, ERC, ERDF, Horizon 2020 and Marie Skłodowska-Curie Actions, European Union; Investissements d'Avenir Labex, Investissements d'Avenir Idex and ANR, France; DFG and AvH Foundation, Germany; Herakleitos, Thales and Aristeia programs cofinanced by EU-ESF and the Greek NSRF, Greece; BSFNSF and GIF, Israel; La Caixa Banking Foundation, CERCA Programme Generalitat de Catalunya and PROMETEO and GenT Programmes Generalitat Valenciana, Spain; Göran Gustafssons Stiftelse, Sweden; The Royal Society and Leverhulme Trust, United Kingdom. The crucial computing support from all WLCG partners is acknowledged gratefully, in particular from CERN, the ATLAS Tier-1 facilities at TRIUMF (Canada), NDGF (Denmark, Norway, Sweden), CC-IN2P3 (France), 
KIT/GridKA (Germany), INFN-CNAF (Italy), NL-T1 (Netherlands), PIC (Spain), ASGC (Taiwan), RAL (UK) and BNL (U.S.), the Tier-2 facilities worldwide, and large non-WLCG resource providers. Major contributors of computing resources are listed in Ref. [99]. We are grateful to the LHC optics, collimation, machine protection, and operations groups that enabled the use of the ATLAS Forward Proton spectrometer.

[1] G. Breit and J. A. Wheeler, Collision of two light quanta, Phys. Rev. 46, 1087 (1934).

[2] W. Heisenberg and H. Euler, Folgerungen aus der diracschen theorie des positrons, Z. Phys. 98, 714 (1936).

[3] J. Schwinger, On gauge invariance and vacuum polarization, Phys. Rev. 82, 664 (1951).

[4] ATLAS Collaboration, Measurement of exclusive $\gamma \gamma \rightarrow$ $\ell^{+} \ell^{-}$production in proton-proton collisions at $\sqrt{s}=$ $7 \mathrm{TeV}$ with the ATLAS detector, Phys. Lett. B 749, 242 (2015).

[5] ATLAS Collaboration, Measurement of the exclusive $\gamma \gamma \rightarrow$ $\mu^{+} \mu^{-}$process in proton-proton collisions at $\sqrt{s}=13 \mathrm{TeV}$ with the ATLAS detector, Phys. Lett. B 777, 303 (2018).

[6] CMS Collaboration, Search for exclusive or semi-exclusive $\gamma \gamma$ production and observation of exclusive and semi-exclusive $e^{+} e^{-}$production in $p p$ collisions at $\sqrt{s}=7 \mathrm{TeV}$, J. High Energy Phys. 11 (2012) 080.

[7] CMS Collaboration, Exclusive $\gamma \gamma \rightarrow \mu^{+} \mu^{-}$production in proton-proton collisions at $\sqrt{s}=7 \mathrm{TeV}$, J. High Energy Phys. 01 (2012) 052.

[8] R. J. Gould and G. P. Schréder, Pair production in photonphoton collisions, Phys. Rev. 155, 1404 (1967).

[9] E. Dwek and F. Krennrich, The extragalactic background light and the gamma-ray opacity of the universe, Astropart. Phys. 43, 112 (2013).

[10] R. Ruffini, G. Vereshchagin, and S.-S. Xue, Electronpositron pairs in physics and astrophysics: From heavy nuclei to black holes, Phys. Rep. 487, 1 (2010).

[11] V. M. Kaspi and A. Beloborodov, Magnetars, Annu. Rev. Astron. Astrophys. 55, 261 (2017).

[12] M.-S. Chen, I. J. Muzinich, H. Terazawa, and T. P. Cheng, Lepton pair production from two-photon processes, Phys. Rev. D 7, 3485 (1973).

[13] V. M. Budnev, I. F. Ginzburg, G. V. Meledin, and V. G. Serbo, The two-photon particle production mechanism. Physical problems. Applications. Equivalent photon approximation, Phys. Rep. 15, 181 (1975).

[14] K. Piotrzkowski, Tagging two-photon production at the CERN Large Hadron Collider, Phys. Rev. D 63, 071502 (2001).

[15] V. A. Khoze, A. D. Martin, and M. G. Ryskin, Prospects for new physics observations in diffractive processes at the LHC and Tevatron, Eur. Phys. J. C 23, 311 (2002).

[16] J. de Favereau de Jeneret et al., High energy photon interactions at the LHC, arXiv:0908.2020.

[17] LHCb Collaboration, Central exclusive production of $J / \psi$ and $\psi(2 S)$ mesons in $p p$ collisions at $\sqrt{s}=13 \mathrm{TeV}$, J. High Energy Phys. 10 (2018) 167.
[18] ATLAS Collaboration, Observation of CentralityDependent Acoplanarity for Muon Pairs Produced via Two-Photon Scattering in $\mathrm{Pb}+\mathrm{Pb}$ Collisions at $\sqrt{s_{\mathrm{NN}}}=$ 5.02 TeV with the ATLAS Detector, Phys. Rev. Lett. 121, 212301 (2018).

[19] ATLAS Collaboration, Observation of Light-by-Light Scattering in Ultraperipheral $\mathrm{Pb}+\mathrm{Pb}$ Collisions with the ATLAS Detector, Phys. Rev. Lett. 123, 052001 (2019).

[20] CMS Collaboration, Evidence for light-by-light scattering and searches for axion-like particles in ultraperipheral $\mathrm{PbPb}$ collisions at $\sqrt{s_{\mathrm{NN}}}=5.02 \mathrm{TeV}$, Phys. Lett. B 797, 134826 (2019).

[21] ALICE Collaboration, Coherent $J / \psi$ photoproduction at forward rapidity in ultra-peripheral $\mathrm{Pb}-\mathrm{Pb}$ collisions at $\sqrt{s_{\mathrm{NN}}}=5.02 \mathrm{TeV}$, Phys. Lett. B 798, 134926 (2019).

[22] A. Baltz, The physics of ultraperipheral collisions at the LHC, Phys. Rep. 458, 1 (2008).

[23] PHENIX Collaboration, Photoproduction of $J / \psi$ and of high mass $e^{+} e^{-}$in ultra-peripheral $\mathrm{Au}+\mathrm{Au}$ collisions at $\sqrt{s}=200 \mathrm{GeV}$, Phys. Lett. B 679, 321 (2009).

[24] STAR Collaboration, Probing extreme electromagnetic fields with the Breit-Wheeler process, arXiv:1910.12400.

[25] L. Beresford and J. Liu, New physics and tau $g-2$ using LHC heavy ion collisions, arXiv:1908.05180 [Phys. Rev. D (to be published)].

[26] M. Dyndal, M. Klusek-Gawenda, M. Schott, and A. Szczurek, Anomalous electromagnetic moments of $\tau$ lepton in $\gamma \gamma \rightarrow \tau^{+} \tau^{-}$reaction in $\mathrm{Pb}+\mathrm{Pb}$ collisions at the $\mathrm{LHC}$, Phys. Lett. B 809, 135682 (2020).

[27] D. Burke et al., Positron Production in Multiphoton Lightby-Light Scattering, Phys. Rev. Lett. 79, 1626 (1997).

[28] M. Ruf, G. R. Mocken, C. Müller, K. Z. Hatsagortsyan, and C. H. Keitel, Pair Production in Laser Fields Oscillating in Space and Time, Phys. Rev. Lett. 102, 080402 (2009).

[29] M. Altarelli et al., Summary of strong-field QED Workshop, arXiv:1905.00059.

[30] H. Abramowicz et al., Letter of intent for the LUXE experiment, arXiv:1909.00860.

[31] CMS Collaboration, Observation of proton-tagged, central (semi)exclusive production of high-mass lepton pairs in $p p$ collisions at $13 \mathrm{TeV}$ with the CMS-TOTEM precision proton spectrometer, J. High Energy Phys. 07 (2018) 153.

[32] V. Khoze, A. Martin, and M. Ryskin, Diffraction at the LHC, Eur. Phys. J. C 73, 2503 (2013).

[33] L. A. Harland-Lang, V. A. Khoze, and M. G. Ryskin, The photon PDF in events with rapidity gaps, Eur. Phys. J. C 76, 255 (2016).

[34] M. Dyndal and L. Schoeffel, The role of finite-size effects on the spectrum of equivalent photons in proton-proton collisions at the LHC, Phys. Lett. B 741, 66 (2015).

[35] L. Harland-Lang, V. Khoze, and M. Ryskin, Exclusive physics at the LHC with SUPERCHIC 2, Eur. Phys. J. C 76, 9 (2016).

[36] B. Cox, F. Loebinger, and A. Pilkington, Detecting Higgs bosons in the $b \bar{b}$ decay channel using forward proton tagging at the LHC, J. High Energy Phys. 10 (2007) 090.

[37] M. G. Albrow et al., The FP420 R\&D project: Higgs and new physics with forward protons at the LHC, J. Instrum. 4, T10001 (2009). 
[38] M. Trzebiński, R. Staszewski, and J. Chwastowski, On the possibility of measuring the single-tagged exclusive jets at the LHC, Eur. Phys. J. C 75, 320 (2015).

[39] S. Tizchang and S. M. Etesami, Pinning down the gauge boson couplings in $W W \gamma$ production using forward proton tagging, J. High Energy Phys. 07 (2020) 191.

[40] ATLAS Collaboration, Measurement of exclusive $\gamma \gamma \rightarrow W^{+} W^{-}$production and search for exclusive Higgs boson production in $p p$ collisions at $\sqrt{s}=8 \mathrm{TeV}$ using the ATLAS detector, Phys. Rev. D 94, 032011 (2016).

[41] CMS Collaboration, Evidence for exclusive $\gamma \gamma \rightarrow W^{+} W^{-}$ production and constraints on anomalous quartic gauge couplings in $p p$ collisions at $\sqrt{s}=7$ and $8 \mathrm{TeV}$, J. High Energy Phys. 08 (2016) 119.

[42] S. Heinemeyer, V. A. Khoze, M. G. Ryskin, W. J. Stirling, M. Tasevsky, and G. Weiglein, Studying the MSSM Higgs sector by forward proton tagging at the LHC, Eur. Phys. J. C 53, 231 (2008).

[43] L. A. Harland-Lang, C. H. Kom, K. Sakurai, and W. J. Stirling, Measuring the masses of a pair of semi-invisibly decaying particles in central exclusive production with forward proton tagging, Eur. Phys. J. C 72, 1969 (2012).

[44] C. Baldenegro, S. Fichet, G. von Gersdorff, and C. Royon, Searching for axion-like particles with proton tagging at the LHC, J. High Energy Phys. 06 (2018) 131.

[45] L. Beresford and J. Liu, Search Strategy for Sleptons and Dark Matter Using the LHC as a Photon Collider, Phys. Rev. Lett. 123, 141801 (2019).

[46] L. A. Harland-Lang, V. A. Khoze, M. G. Ryskin, and M. Tasevsky, LHC searches for dark matter in compressed mass scenarios: Challenges in the forward proton mode, J. High Energy Phys. 04 (2019) 010.

[47] ATLAS Collaboration, The ATLAS experiment at the CERN Large Hadron Collider, J. Instrum. 3, S08003 (2008).

[48] ATLAS Collaboration, ATLAS insertable $B$-layer technical design report, CERN Report No. ATLAS-TDR-19; CERN-LHCC-2010-013, 2010, https://cds.cern.ch/record/ 1291633.

[49] B. Abbott et al., Production and integration of the ATLAS insertable $B$-layer, J. Instrum. 13, T05008 (2018).

[50] ATLAS uses a right-handed coordinate system with its origin at the nominal interaction point (IP) in the center of the detector and the $z$ axis along the beam pipe. The $x$ axis points from the IP to the center of the LHC ring, and the $y$ axis points upward. Cylindrical coordinates $(r, \phi)$ are used in the transverse plane, $\phi$ being the azimuthal angle around the $z$ axis. The pseudorapidity is defined in terms of the polar angle $\theta$ as $\eta=-\ln \tan (\theta / 2)$. The transverse momentum is denoted $p_{T}$. Angular distances are measured in units of $\Delta R=\sqrt{(\Delta \eta)^{2}+(\Delta \phi)^{2}}$. Rapidity is defined as $y=\frac{1}{2} \ln \left[\left(E+p_{z}\right) /\left(E-p_{z}\right)\right]$, where $E$ is the energy and $p_{z}$ is the longitudinal component of the momentum of the particle.

[51] ATLAS Collaboration, Performance of the ATLAS trigger system in 2015, Eur. Phys. J. C 77, 317 (2017).

[52] ATLAS Collaboration, Trigger menu in 2017, CERN Report No. ATL-DAQ-PUB-2018-002, 2018, https://cds .cern.ch/record/2625986.
[53] ATLAS Collaboration, Performance of the ATLAS muon triggers in run 2, J. Instrum. 15, P09015 (2020).

[54] ATLAS Collaboration, Performance of electron and photon triggers in ATLAS during LHC run 2, Eur. Phys. J. C 80, 47 (2020).

[55] ATLAS Collaboration, ATLAS data quality operations and performance for 2015-2018 data-taking, J. Instrum. 15, P04003 (2020).

[56] ATLAS Collaboration, Technical design report for the ATLAS forward proton detector, CERN Tech. Report No. CERN-LHCC-2015-009, ATLAS-TDR-024, 2015, https://cds.cern.ch/record/2017378.

[57] ATLAS Collaboration, Proton tagging with the one arm AFP detector, CERN Report No. ATL-PHYS-PUB-2017012, 2017, https://cds.cern.ch/record/2273274.

[58] J. Lange, E. Cavallaro, S. Grinstein, and I. L. Paz, 3D silicon pixel detectors for the ATLAS forward physics experiment, J. Instrum. 10, C03031 (2015).

[59] J. Lange et al., Beam tests of an integrated prototype of the ATLAS forward proton detector, J. Instrum. 11, P09005 (2016).

[60] M. Garcia-Sciveres et al., The FE-I4 pixel readout integrated circuit, Nucl. Instrum. Methods Phys. Res., Sect. A 636, S155 (2011).

[61] V. Zivkovic et al., The FE-I4 pixel readout system-on-chip resubmission for the insertable $B$-layer project, J. Instrum. 7, C02050 (2012).

[62] S. Grinstein et al., Module production of the one-arm AFP 3D pixel tracker, J. Instrum. 12, C01086 (2017).

[63] M. Kocian, Readout and trigger for the AFP detector at ATLAS experiment, J. Instrum. 12, C01077 (2017).

[64] M. Bahr et al., HERWIG ++ physics and manual, Eur. Phys. J. C 58, 639 (2008).

[65] J. Bellm et al., Herwig 7.0/HERWIG ++3.0 release note, Eur. Phys. J. C 76, 196 (2016).

[66] J. Vermaseren, Two photon processes at very high energies, Nucl. Phys. B229, 347 (1983).

[67] F. W. Brasse, W. Flauger, J. Gayler, S. P. Goel, R. Haidan, M. Merkwitz, and H. Wriedt, Parametrization of the $q^{2}$ dependence of $\gamma_{V} p$ total cross sections in the resonance region, Nucl. Phys. B110, 413 (1976).

[68] A. Suri and D. R. Yennie, The space-time phenomenology of photon absorption and inelastic electron scattering, Ann. Phys. (N.Y.) 72, 243 (1972).

[69] T. Sjöstrand, High-energy-physics event generation with PYTHIA 5.7 and JETSET7.4, Comput. Phys. Commun. 82, 74 (1994).

[70] B. Andersson, G. Gustafson, G. Ingelman, and T. Sjöstrand, Parton fragmentation and string dynamics, Phys. Rep. 97, 31 (1983).

[71] S. Agostinelli et al., GEANT4-A simulation toolkit, Nucl. Instrum. Methods Phys. Res., Sect. A 506, 250 (2003).

[72] ATLAS Collaboration, The ATLAS simulation infrastructure, Eur. Phys. J. C 70, 823 (2010).

[73] ATLAS Collaboration, The simulation principle and performance of the ATLAS fast calorimeter simulation FastCaloSim, CERN Report No. ATL-PHYS-PUB-2010-013, 2010, https://cds.cern.ch/record/1300517.

[74] ATLAS Collaboration, Early inner detector tracking performance in the 2015 data at $\sqrt{s}=13 \mathrm{TeV}$, CERN Report 
No. ATL-PHYS-PUB-2015-051, 2015, https://cds.cern.ch/ record/2110140.

[75] ATLAS Collaboration, Performance of the ATLAS track reconstruction algorithms in dense environments in LHC run 2, Eur. Phys. J. C 77, 673 (2017).

[76] ATLAS Collaboration, Electron reconstruction and identification in the ATLAS experiment using the 2015 and 2016 LHC proton-proton collision data at $\sqrt{s}=13 \mathrm{TeV}$, Eur. Phys. J. C 79, 639 (2019).

[77] ATLAS Collaboration, Muon reconstruction performance of the ATLAS detector in proton-proton collision data at $\sqrt{s}=13 \mathrm{TeV}$, Eur. Phys. J. C 76, 292 (2016).

[78] $z_{0}$ is the longitudinal impact parameter relative to the primary vertex, where the primary vertex is defined as the vertex with the largest $\sum p_{T}^{2}$ of associated tracks.

[79] ATLAS Collaboration, Searches for electroweak production of supersymmetric particles with compressed mass spectra in $\sqrt{s}=13 \mathrm{TeV} p p$ collisions with the ATLAS detector, Phys. Rev. D 101, 052005 (2020).

[80] ATLAS Collaboration, Search for electroweak production of charginos and sleptons decaying into final states with two leptons and missing transverse momentum in $\sqrt{s}=13 \mathrm{TeV}$ $p p$ collisions using the ATLAS detector, Eur. Phys. J. C 80, 123 (2020).

[81] L. Evans and P. Bryant, LHC machine, J. Instrum. 3, S08001 (2008).

[82] LHC Optics Working Group, LHC optics web home, http:// abpdata.web.cern.ch/abpdata/lhc_optics_web/www/.

[83] The function $T\left(\xi_{\mathrm{AFP}}\right)=a \xi_{\mathrm{AFP}}+b \xi_{\mathrm{AFP}}{ }^{2}$ with $a=-119$ and $b=-164 \mathrm{~mm}$ provides an approximate parametrization.

[84] W. Herr and F. Schmidt, A MAD-X primer, CERN Report No. CERN-AB-2004-027-ABP, 2004, p. 32, https://cds.cern .ch/record/744163.

[85] L. Deniau, H. Grote, G. Roy, and F. Schmidt, The MAD-X program user's reference manual, http://madx.web.cern.ch/ madx/releases/last-rel/madxuguide.pdf.

[86] R. Staszewski and J. Chwastowski, Transport simulation and diffractive event reconstruction at the LHC, Nucl. Instrum. Methods Phys. Res., Sect. A 609, 136 (2009).

[87] R. Staszewski, J. Chwastowski, K. Korcyl, and M. Trzebiński, Alignment-related effects in forward proton experiments at the LHC, Nucl. Instrum. Methods Phys. Res., Sect. A 801, 34 (2015).

[88] G. Valentino, R. Aßmann, R. Bruce, S. Redaelli, A. Rossi, N. Sammut, and D. Wollmann, Semiautomatic beam-based
LHC collimator alignment, Phys. Rev. ST Accel. Beams 15, 051002 (2012).

[89] C. Zamantzas et al., The LHC beam loss monitoring system's data contribution to other systems, IEEE Nucl. Sci. Symp. Conf. Record 3, 2331 (2007).

[90] G. Valentino et al., Final implementation, commissioning, and performance of embedded collimator beam position monitors in the large hadron collider, Phys. Rev. Accel. Beams 20, 081002 (2017).

[91] G. Cowan, K. Cranmer, E. Gross, and O. Vitells, Asymptotic formulae for likelihood-based tests of new physics, Eur. Phys. J. C 71, 1554 (2011); Erratum, Eur. Phys. J. C 73, 2501 (2013).

[92] M. Baak, G. J. Besjes, D. Côté, A. Koutsman, J. Lorenz, and D. Short, HistFitter software framework for statistical data analysis, Eur. Phys. J. C 75, 153 (2015).

[93] The statistical significance in the $e e+p(\mu \mu+p)$ final state corresponds to $9.7 \sigma(13 \sigma)$.

[94] Exactly two same-flavor opposite-charge Born leptons with $p_{T}(e / \mu)>18 / 15 \mathrm{GeV},|\eta(e / \mu)|<2.47 / 2.4, p_{T}^{\ell \ell}<5 \mathrm{GeV}$, $A_{\phi}^{\ell \ell}<0.01, m_{\ell \ell}>20 \mathrm{GeV}, m_{\ell \ell} \notin[70,105] \mathrm{GeV}, \xi_{\ell \ell}^{A} \in$ $[0.035,0.08]$ or $\xi_{\ell \ell}^{C} \in[0.035,0.08]$, no charged particles with $p_{T}>500 \mathrm{MeV}$ and $|\eta|<2.5, \geq 1$ forward proton.

[95] G. Avoni et al., The new LUCID-2 detector for luminosity measurement and monitoring in ATLAS, J. Instrum. 13, P07017 (2018).

[96] ATLAS Collaboration, Luminosity determination in $p p$ collisions at $\sqrt{s}=13 \mathrm{TeV}$ using the ATLAS detector at the LHC, CERN Tech. Report No. Atlas-CONF-2019-021, CERN, 2019, https://cds.cern.ch/record/2677054.

[97] L. Harland-Lang, M. Tasevsky, V. Khoze, and M. Ryskin, A new approach to modelling elastic and inelastic photoninitiated production at the LHC: SuperChic 4, Eur. Phys. J. C 80, 925 (2020).

[98] Uncertainties on predicted soft-survival factors are estimated in accord with Ref. [33]. For the exclusive process, the uncertainty on $S_{\text {surv }}$ is estimated by the $m_{\ell \ell}$ variations, while for the single-dissociative process, the uncertainty on $S_{\text {surv }}$ is estimated by taking the difference in $S_{\text {surv }}$ between the exclusive and single-dissociative processes.

[99] ATLAS Collaboration, ATLAS computing acknowledgements, CERN Report No. ATL-SOFT-PUB-2020-001, https://cds.cern.ch/record/2717821.

G. Aad, ${ }^{102}$ B. Abbott, ${ }^{128}$ D. C. Abbott, ${ }^{103}$ A. Abed Abud, ${ }^{36}$ K. Abeling, ${ }^{53}$ D. K. Abhayasinghe, ${ }^{94}$ S. H. Abidi, ${ }^{167}$ O. S. AbouZeid, ${ }^{40}$ N. L. Abraham, ${ }^{156}$ H. Abramowicz, ${ }^{161}$ H. Abreu, ${ }^{160}$ Y. Abulaiti, ${ }^{6}$ B. S. Acharya, ${ }^{67 a, 67 b, b}$ B. Achkar, ${ }^{53}$ L. Adam, ${ }^{100}$ C. Adam Bourdarios, ${ }^{5}$ L. Adamczyk, ${ }^{84 a}$ L. Adamek, ${ }^{167}$ J. Adelman, ${ }^{121}$ A. Adiguzel,${ }^{12 \mathrm{c}}$ S. Adorni,${ }^{54}$ T. Adye,${ }^{143}$ A. A. Affolder, ${ }^{145}$ Y. Afik, ${ }^{160}$ C. Agapopoulou, ${ }^{65}$ M. N. Agaras, ${ }^{38}$ A. Aggarwal, ${ }^{119}$ C. Agheorghiesei, ${ }^{27 c}$ J. A. Aguilar-Saavedra, ${ }^{139 f, 139 a, c}$ A. Ahmad, ${ }^{36}$ F. Ahmadov, ${ }^{80}$ W. S. Ahmed, ${ }^{104}$ X. Ai, ${ }^{18}$ G. Aielli, ${ }^{74 a, 74 b}$ S. Akatsuka ${ }^{86}$ M. Akbiyik, ${ }^{100}$ T. P. A. Åkesson, ${ }^{97}$ E. Akilli, ${ }^{54}$ A. V. Akimov ${ }^{111}$ K. Al Khoury, ${ }^{65}$ G. L. Alberghi, ${ }^{23 b, 23 a}$ J. Albert, ${ }^{176}$ M. J. Alconada Verzini, ${ }^{161}$ S. Alderweireldt, ${ }^{36}$ M. Aleksa ${ }^{36}$ I. N. Aleksandrov, ${ }^{80}$ C. Alexa, ${ }^{27 b}$ T. Alexopoulos,${ }^{10}$ A. Alfonsi, ${ }^{120}$ F. Alfonsi, ${ }^{23 b, 23 a}$ M. Alhroob, ${ }^{128}$ B. Ali, ${ }^{141}$ S. Ali,${ }^{158}$ M. Aliev, ${ }^{166}$ G. Alimonti, ${ }^{69 a}$ C. Allaire, ${ }^{36}$ B. M. M. Allbrooke, ${ }^{156}$ B. W. Allen, ${ }^{131}$ P. P. Allport, ${ }^{21}$ A. Aloisio, ${ }^{70 a, 70 b}$ F. Alonso, ${ }^{89}$ C. Alpigiani, ${ }^{148}$ 


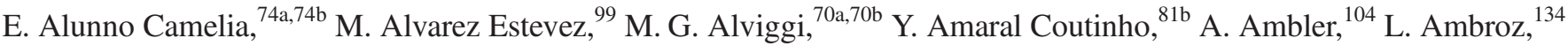

C. Amelung, ${ }^{36}$ D. Amidei, ${ }^{106}$ S. P. Amor Dos Santos, ${ }^{139 a}$ S. Amoroso, ${ }^{46}$ C. S. Amrouche, ${ }^{54}$ F. An,${ }^{79}$ C. Anastopoulos, ${ }^{149}$ N. Andari, ${ }^{144}$ T. Andeen, ${ }^{11}$ J. K. Anders, ${ }^{20}$ S. Y. Andrean, ${ }^{45 a, 45 b}$ A. Andreazza, ${ }^{69 a, 69 b}$ V. Andrei, ${ }^{61 \mathrm{a}}$ C. R. Anelli, ${ }^{176}$ S. Angelidakis, ${ }^{9}$ A. Angerami, ${ }^{39}$ A. V. Anisenkov, ${ }^{122 b, 122 a}$ A. Annovi, ${ }^{72 a}$ C. Antel, ${ }^{54}$ M. T. Anthony, ${ }^{149}$ E. Antipov, ${ }^{129}$ M. Antonelli, ${ }^{51}$ D. J. A. Antrim, ${ }^{18}$ F. Anulli, ${ }^{73 a}$ M. Aoki, ${ }^{82}$ J. A. Aparisi Pozo,${ }^{174}$ M. A. Aparo,${ }^{156}$ L. Aperio Bella, ${ }^{46}$ N. Aranzabal, ${ }^{36}$ V. Araujo Ferraz, ${ }^{81 a}$ R. Araujo Pereira, ${ }^{81 b}$ C. Arcangeletti ${ }^{51}$ A. T. H. Arce, ${ }^{49}$ J-F. Arguin, ${ }^{110}$ S. Argyropoulos, ${ }^{52}$ J.-H. Arling, ${ }^{46}$ A. J. Armbruster ${ }^{36}$ A. Armstrong, ${ }^{171}$ O. Arnaez, ${ }^{167}$ H. Arnold, ${ }^{120}$ Z. P. Arrubarrena Tame,${ }^{114}$ G. Artoni, ${ }^{134}$ H. Asada,${ }^{117}$ K. Asai, ${ }^{126}$ S. Asai, ${ }^{163}$ T. Asawatavonvanich, ${ }^{165}$ N. Asbah, ${ }^{59}$ E. M. Asimakopoulou, ${ }^{172}$ L. Asquith, ${ }^{156}$ J. Assahsah, ${ }^{35 \mathrm{~d}}$ K. Assamagan, ${ }^{29}$ R. Astalos ${ }^{28 \mathrm{a}}$ R. J. Atkin, ${ }^{33 \mathrm{a}}$ M. Atkinson, ${ }^{173}$ N. B. Atlay, ${ }^{19}$ H. Atmani, ${ }^{65}$ P. A. Atmasiddha, ${ }^{106}$ K. Augsten, ${ }^{141}$ V. A. Austrup,${ }^{182}$ G. Avolio, ${ }^{36}$ M. K. Ayoub, ${ }^{15 a}$ G. Azuelos, ${ }^{110, \mathrm{~d}}$ D. Babal,${ }^{28 \mathrm{a}} \mathrm{H}$. Bachacou, ${ }^{144} \mathrm{~K}$. Bachas, ${ }^{162}$ F. Backman,${ }^{45 a, 45 b}$ P. Bagnaia, ${ }^{73 a, 73 \mathrm{~b}} \mathrm{M}$. Bahmani, ${ }^{85}$ H. Bahrasemani, ${ }^{152}$ A. J. Bailey, ${ }^{174}$ V. R. Bailey, ${ }^{173}$ J. T. Baines,${ }^{143}$ C. Bakalis,${ }^{10}$ O. K. Baker, ${ }^{183}$ P. J. Bakker, ${ }^{120}$ E. Bakos,${ }^{16}$ D. Bakshi Gupta, ${ }^{8}$ S. Balaji, ${ }^{157}$ R. Balasubramanian, ${ }^{120}$ E. M. Baldin, ${ }^{122 b, 122 a}$ P. Balek ${ }^{180}$ F. Balli, ${ }^{144}$ W. K. Balunas, ${ }^{134}$ J. Balz, ${ }^{100}$ E. Banas, ${ }^{85}$ M. Bandieramonte, ${ }^{138}$ A. Bandyopadhyay, ${ }^{19}$ Sw. Banerjee,${ }^{181, \mathrm{e}}$ L. Barak, ${ }^{161}$ W. M. Barbe,${ }^{38}$ E. L. Barberio, ${ }^{105}$ D. Barberis, ${ }^{55 b, 55 a}$ M. Barbero, ${ }^{102}$ G. Barbour, ${ }^{95}$ T. Barillari, ${ }^{115}$ M-S. Barisits,${ }^{36}$ J. Barkeloo, ${ }^{131}$ T. Barklow, ${ }^{153}$ R. Barnea, ${ }^{160}$ B. M. Barnett, ${ }^{143}$ R. M. Barnett, ${ }^{18}$ Z. Barnovska-Blenessy, ${ }^{60 a}$ A. Baroncelli, ${ }^{60 a}$ G. Barone, ${ }^{29}$ A. J. Barr, ${ }^{134}$ L. Barranco Navarro, ${ }^{45 a, 45 b}$ F. Barreiro, ${ }^{99}$ J. Barreiro Guimarães da Costa, ${ }^{15 a}$ U. Barron, ${ }^{161}$ S. Barsov, ${ }^{137}$ F. Bartels, ${ }^{61 a}$ R. Bartoldus, ${ }^{153}$ G. Bartolini, ${ }^{102}$ A. E. Barton, ${ }^{90}$ P. Bartos, ${ }^{28 a}$ A. Basalaev,${ }^{46}$ A. Basan, ${ }^{100}$ A. Bassalat, ${ }^{65, f}$ M. J. Basso, ${ }^{167}$ R. L. Bates, ${ }^{57}$ S. Batlamous, ${ }^{35 e}$ J. R. Batley, ${ }^{32}$ B. Batool, ${ }^{151}$ M. Battaglia, ${ }^{145}$ M. Bauce,${ }^{73 a, 73 b}$ F. Bauer, ${ }^{144}$ P. Bauer, ${ }^{24}$ H. S. Bawa, ${ }^{31}$ A. Bayirli, ${ }^{12 c}$ J. B. Beacham, ${ }^{49}$ T. Beau, ${ }^{135}$ P. H. Beauchemin, ${ }^{170}$ F. Becherer, ${ }^{52}$ P. Bechtle, ${ }^{24}$ H. C. Beck, ${ }^{53}$ H. P. Beck, ${ }^{20, g}$ K. Becker ${ }^{178}$ C. Becot, ${ }^{46}$ A. Beddall, ${ }^{12 \mathrm{~d}}$ A. J. Beddall, ${ }^{12 \mathrm{a}}$ V. A. Bednyakov, ${ }^{80}$ M. Bedognetti, ${ }^{120}$ C. P. Bee, ${ }^{155}$ T. A. Beermann, ${ }^{182}$ M. Begalli, ${ }^{81 b}$ M. Begel, ${ }^{29}$ A. Behera, ${ }^{155}$ J. K. Behr, ${ }^{46}$ F. Beisiegel, ${ }^{24}$ M. Belfkir, ${ }^{5}$ A. S. Bell,${ }^{95}$ G. Bella,${ }^{161}$ L. Bellagamba, ${ }^{23 b}$ A. Bellerive, ${ }^{34}$ P. Bellos, ${ }^{9}$ K. Beloborodov, ${ }^{122 b, 122 a}$ K. Belotskiy, ${ }^{112}$ N. L. Belyaev, ${ }^{112}$ D. Benchekroun, ${ }^{35 a}$ N. Benekos, ${ }^{10}$ Y. Benhammou, ${ }^{161}$ D. P. Benjamin, ${ }^{6}$ M. Benoit, ${ }^{29}$ J. R. Bensinger ${ }^{26}$ S. Bentvelsen, ${ }^{120}$ L. Beresford, ${ }^{134}$ M. Beretta, ${ }^{51}$ D. Berge,${ }^{19}$ E. Bergeaas Kuutmann, ${ }^{172}$ N. Berger, ${ }^{5}$ B. Bergmann, ${ }^{141}$ L. J. Bergsten, ${ }^{26}$ J. Beringer, ${ }^{18}$ S. Berlendis, ${ }^{7}$ G. Bernardi, ${ }^{135}$ C. Bernius, ${ }^{153}$ F. U. Bernlochner ${ }^{24}$ T. Berry, ${ }^{94}$ P. Berta, ${ }^{100}$ A. Berthold, ${ }^{48}$ I. A. Bertram, ${ }^{90}$ O. Bessidskaia Bylund, ${ }^{182}$ N. Besson, ${ }^{144}$ S. Bethke, ${ }^{115}$ A. Betti, ${ }^{42}$ A. J. Bevan, ${ }^{93}$ J. Beyer, ${ }^{115}$ S. Bhatta, ${ }^{155}$ D. S. Bhattacharya,${ }^{177}$ P. Bhattarai, ${ }^{26}$ V. S. Bhopatkar, ${ }^{6}$ R. Bi,${ }^{138}$ R. M. Bianchi, ${ }^{138}$ O. Biebel, ${ }^{114}$

D. Biedermann, ${ }^{19}$ R. Bielski, ${ }^{36}$ K. Bierwagen, ${ }^{100}$ N. V. Biesuz, ${ }^{72 a, 72 b}$ M. Biglietti, ${ }^{75 a}$ T. R. V. Billoud,${ }^{141}$ M. Bindi, ${ }^{53}$ A. Bingul, ${ }^{12 \mathrm{~d}}$ C. Bini, ${ }^{73 a, 73 b}$ S. Biondi, ${ }^{23 b, 23 \mathrm{a}}$ C. J. Birch-sykes, ${ }^{101}$ M. Birman, ${ }^{180}$ T. Bisanz, ${ }^{36}$ J. P. Biswal, ${ }^{3}$ D. Biswas, ${ }^{181, e}$ A. Bitadze, ${ }^{101}$ C. Bittrich ${ }^{48}$ K. Bjørke, ${ }^{133}$ T. Blazek, ${ }^{28 a}$ I. Bloch, ${ }^{46}$ C. Blocker, ${ }^{26}$ A. Blue,${ }^{57}$ U. Blumenschein,${ }^{93}$ G. J. Bobbink, ${ }^{120}$ V. S. Bobrovnikov, ${ }^{122 b, 122 a}$ S. S. Bocchetta,${ }^{97}$ D. Bogavac,${ }^{14}$ A. G. Bogdanchikov, ${ }^{122 b, 122 a}$ C. Bohm, ${ }^{45 a}$ V. Boisvert, ${ }^{94}$ P. Bokan, ${ }^{172,53}$ T. Bold, ${ }^{84 a}$ A. E. Bolz, ${ }^{61 b}$ M. Bomben, ${ }^{135}$ M. Bona, ${ }^{93}$ J. S. Bonilla, ${ }^{131}$ M. Boonekamp, ${ }^{144}$ C. D. Booth, ${ }^{94}$ A. G. Borbély, ${ }^{57}$ H. M. Borecka-Bielska, ${ }^{91}$ L. S. Borgna,${ }^{95}$ A. Borisov, ${ }^{123}$ G. Borissov, ${ }^{90}$ D. Bortoletto, ${ }^{134}$ D. Boscherini, ${ }^{23 \mathrm{~b}}$ M. Bosman, ${ }^{14}$ J. D. Bossio Sola, ${ }^{104}$ K. Bouaouda, ${ }^{35 a}$ J. Boudreau, ${ }^{138}$ E. V. Bouhova-Thacker, ${ }^{90}$ D. Boumediene, ${ }^{38}$ A. Boveia, ${ }^{127}$ J. Boyd, ${ }^{36}$ D. Boye ${ }^{33 c}$ I. R. Boyko, ${ }^{80}$ A. J. Bozson, ${ }^{94}$ J. Bracinik, ${ }^{21}$ N. Brahimi, ${ }^{60 d}$ G. Brandt, ${ }^{182}$ O. Brandt, ${ }^{32}$ F. Braren, ${ }^{46}$ B. Brau, ${ }^{103}$ J. E. Brau, ${ }^{131}$ W. D. Breaden Madden, ${ }^{57}$ K. Brendlinger, ${ }^{46}$ R. Brener, ${ }^{160}$ L. Brenner, ${ }^{36}$ R. Brenner, ${ }^{172}$ S. Bressler, ${ }^{180}$ B. Brickwedde, ${ }^{100}$ D. L. Briglin, ${ }^{21}$ D. Britton, ${ }^{57}$ D. Britzger, ${ }^{115}$ I. Brock, ${ }^{24}$ R. Brock, ${ }^{107}$ G. Brooijmans, ${ }^{39}$ W. K. Brooks, ${ }^{146 \mathrm{~d}}$ E. Brost,${ }^{29}$ P. A. Bruckman de Renstrom, ${ }^{85}$ B. Brüers,${ }^{46}$ D. Bruncko, ${ }^{28 b}$ A. Bruni, ${ }^{23 b}$ G. Bruni, ${ }^{23 b}$ M. Bruschi, ${ }^{23 b}$ N. Bruscino, ${ }^{73 a, 73 b}$ L. Bryngemark, ${ }^{153}$ T. Buanes, ${ }^{17}$ Q. Buat, ${ }^{155}$ P. Buchholz, ${ }^{151}$ A. G. Buckley, ${ }^{57}$ I. A. Budagov, ${ }^{80}$ M. K. Bugge, ${ }^{133}$ O. Bulekov, ${ }^{112}$ B. A. Bullard, ${ }^{59}$ T. J. Burch, ${ }^{121}$ S. Burdin, ${ }^{91}$ C. D. Burgard, ${ }^{120}$ A. M. Burger, ${ }^{129}$ B. Burghgrave, ${ }^{8}$ J. T. P. Burr, ${ }^{46}$ C. D. Burton, ${ }^{11}$ J. C. Burzynski, ${ }^{103}$ V. Büscher, ${ }^{100}$ E. Buschmann, ${ }^{53}$ P. J. Bussey,${ }^{57}$ J. M. Butler, ${ }^{25}$ C. M. Buttar ${ }^{57}$ J. M. Butterworth, ${ }^{95}$ P. Butti, ${ }^{36}$ W. Buttinger,${ }^{143}$ C. J. Buxo Vazquez, ${ }^{107}$ A. Buzatu, ${ }^{158}$ A. R. Buzykaev, ${ }^{122 b, 122 a}$ G. Cabras,${ }^{23 b, 23 a}$ S. Cabrera Urbán, ${ }^{174}$ D. Caforio, ${ }^{56}$ H. Cai, ${ }^{138}$ V. M. M. Cairo, ${ }^{153}$ O. Cakir, ${ }^{4 a}$ N. Calace, ${ }^{36}$ P. Calafiura, ${ }^{18}$ G. Calderini, ${ }^{135}$ P. Calfayan, ${ }^{66}$ G. Callea, ${ }^{57}$ L. P. Caloba, ${ }^{81 b}$ A. Caltabiano, ${ }^{74 a, 74 b}$ S. Calvente Lopez, ${ }^{99}$ D. Calvet, ${ }^{38}$ S. Calvet, ${ }^{38}$ T. P. Calvet, ${ }^{102}$ M. Calvetti, ${ }^{72 a, 72 b}$ R. Camacho Toro, ${ }^{135}$ S. Camarda, ${ }^{36}$ D. Camarero Munoz, ${ }^{99}$ P. Camarri, ${ }^{74 a, 74 b}$ M. T. Camerlingo, ${ }^{75 a, 75 b}$ D. Cameron, ${ }^{133}$ C. Camincher, ${ }^{36}$ S. Campana ${ }^{36}$ M. Campanelli, ${ }^{95}$ A. Camplani, ${ }^{40}$ V. Canale $,{ }^{70 a}, 70 \mathrm{~b}$ A. Canesse,${ }^{104}$ M. Cano Bret,${ }^{78}$ J. Cantero, ${ }^{129}$ T. Cao, ${ }^{161}$ Y. Cao, ${ }^{173}$ M. Capua, ${ }^{41 b, 41 a}$ R. Cardarelli, ${ }^{74 a}$ F. Cardillo, ${ }^{174}$ G. Carducci, ${ }^{41 b, 41 a}$ I. Carli, ${ }^{142}$ T. Carli, ${ }^{36}$ G. Carlino, ${ }^{70 a}$ 
B. T. Carlson, ${ }^{138}$ E. M. Carlson, ${ }^{176,168 a}$ L. Carminati, ${ }^{69,69 b}$ R. M. D. Carney, ${ }^{153}$ S. Caron, ${ }^{119}$ E. Carquin, ${ }^{146 d}$ S. Carrá, ${ }^{46}$ G. Carratta, ${ }^{23 b, 23 a}$ J. W. S. Carter, ${ }^{167}$ T. M. Carter, ${ }^{50}$ M. P. Casado, ${ }^{14, h}$ A. F. Casha, ${ }^{167}$ E. G. Castiglia, ${ }^{183}$ F. L. Castillo, ${ }^{174}$ L. Castillo Garcia, ${ }^{14}$ V. Castillo Gimenez, ${ }^{174}$ N. F. Castro, ${ }^{139 a, 139 e}$ A. Catinaccio, ${ }^{36}$ J. R. Catmore, ${ }^{133}$ A. Cattai, ${ }^{36}$ V. Cavaliere ${ }^{29}$ V. Cavasinni, ${ }^{72 a, 72 b}$ E. Celebi, ${ }^{12 b}$ F. Celli,${ }^{134}$ K. Cerny,${ }^{130}$ A. S. Cerqueira, ${ }^{81 a}$ A. Cerri, ${ }^{156}$ L. Cerrito, ${ }^{74 a, 74 b}$ F. Cerutti, ${ }^{18}$ A. Cervelli, ${ }^{23 b, 23 a}$ S. A. Cetin, ${ }^{12 b}$ Z. Chadi, ${ }^{35 a}$ D. Chakraborty, ${ }^{121}$ J. Chan, ${ }^{181}$ W. S. Chan, ${ }^{120}$ W. Y. Chan,${ }^{91}$ J. D. Chapman, ${ }^{32}$ B. Chargeishvili, ${ }^{159 b}$ D. G. Charlton, ${ }^{21}$ T. P. Charman, ${ }^{93}$ M. Chatterjee, ${ }^{20}$ C. C. Chau, ${ }^{34}$ S. Che, ${ }^{127}$ S. Chekanov, ${ }^{6}$ S. V. Chekulaev, ${ }^{168 a}$ G. A. Chelkov, ${ }^{80, i}$ B. Chen, ${ }^{79}$ C. Chen, ${ }^{60 a}$ C. H. Chen, ${ }^{79}$ H. Chen, ${ }^{15 \mathrm{c}}$ H. Chen, ${ }^{29}$ J. Chen, ${ }^{60 \mathrm{a}}$ J. Chen, ${ }^{39}$ J. Chen, ${ }^{26}$ S. Chen, ${ }^{136}$ S. J. Chen, ${ }^{15 \mathrm{c}}$ X. Chen, ${ }^{15 \mathrm{~b}}$ Y. Chen, ${ }^{60 \mathrm{a}}$ Y-H. Chen, ${ }^{46}$ H. C. Cheng, ${ }^{63 \mathrm{a}}$ H. J. Cheng, ${ }^{15 a}$ A. Cheplakov, ${ }^{80}$ E. Cheremushkina, ${ }^{123}$ R. Cherkaoui El Moursli, ${ }^{35 e}$ E. Cheu, ${ }^{7}$ K. Cheung, ${ }^{64}$ T. J. A. Chevalérias, ${ }^{144}$ L. Chevalier, ${ }^{144}$ V. Chiarella, ${ }^{51}$ G. Chiarelli, ${ }^{72 a}$ G. Chiodini ${ }^{68 a}$ A. S. Chisholm, ${ }^{21}$ A. Chitan, ${ }^{27 b}$ I. Chiu, ${ }^{163}$ Y. H. Chiu, ${ }^{176}$ M. V. Chizhov, ${ }^{80}$ K. Choi, ${ }^{11}$ A. R. Chomont, ${ }^{73 a, 73 b}$ Y. Chou, ${ }^{103}$ Y. S. Chow, ${ }^{120}$ L. D. Christopher, ${ }^{33 e}$ M. C. Chu ${ }^{63 a}$ X. Chu, ${ }^{15 a, 15 d}$ J. Chudoba, ${ }^{140}$ J. J. Chwastowski,${ }^{85}$ L. Chytka,${ }^{130}$ D. Cieri, ${ }^{115}$ K. M. Ciesla, ${ }^{85}$ V. Cindro, ${ }^{92}$ I. A. Cioară, ${ }^{27 b}$ A. Ciocio, ${ }^{18}$ F. Cirotto, ${ }^{70 a, 70 b}$ Z. H. Citron, ${ }^{180, j}$ M. Citterio, ${ }^{69 a}$ D. A. Ciubotaru, ${ }^{27 b}$ B. M. Ciungu, ${ }^{167}$ A. Clark, ${ }^{54}$ P. J. Clark, ${ }^{50}$ S. E. Clawson, ${ }^{101}$ C. Clement,${ }^{45 a, 45 b}$ L. Clissa, ${ }^{23 b, 23 a}$ Y. Coadou, ${ }^{102}$ M. Cobal,${ }^{67 a, 67 c}$ A. Coccaro, ${ }^{55 b}$ J. Cochran, ${ }^{79}$ R. Coelho Lopes De Sa, ${ }^{103}$ H. Cohen, ${ }^{161}$ A. E. C. Coimbra, ${ }^{36}$ B. Cole, ${ }^{39}$ A. P. Colijn, ${ }^{120}$ J. Collot, ${ }^{58}$ P. Conde Muiño, ${ }^{139 a, 139 h}$ S. H. Connell, ${ }^{33 \mathrm{c}}$ I. A. Connelly, ${ }^{57}$ S. Constantinescu, ${ }^{27 \mathrm{~b}}$ F. Conventi, ${ }^{70 a, \mathrm{k}}$ A. M. Cooper-Sarkar, ${ }^{134}$ F. Cormier, ${ }^{175}$ K. J. R. Cormier, ${ }^{167}$ L. D. Corpe, ${ }^{95}$ M. Corradi,${ }^{73 a, 73 b}$ E. E. Corrigan, ${ }^{97}$ F. Corriveau, ${ }^{104,1}$ M. J. Costa, ${ }^{174}$ F. Costanza, ${ }^{5}$ D. Costanzo, ${ }^{149}$ G. Cowan, ${ }^{94}$ J. W. Cowley, ${ }^{32}$ J. Crane,${ }^{101}$ K. Cranmer, ${ }^{125}$ R. A. Creager, ${ }^{136}$ S. Crépé-Renaudin, ${ }^{58}$ F. Crescioli, ${ }^{135}$ M. Cristinziani, ${ }^{24}$ V. Croft,,${ }^{170}$ G. Crosetti, ${ }^{41 b, 41 a}$ A. Cueto, ${ }^{5}$ T. Cuhadar Donszelmann, ${ }^{171}$ H. Cui, ${ }^{15 a, 15 d}$ A. R. Cukierman, ${ }^{153}$ W. R. Cunningham, ${ }^{57}$ S. Czekierda, ${ }^{85}$ P. Czodrowski, ${ }^{36}$ M. M. Czurylo, ${ }^{61 b}$ M. J. Da Cunha Sargedas De Sousa, ${ }^{60 b}$ J. V. Da Fonseca Pinto, ${ }^{81 b}$ C. Da Via, ${ }^{101}$ W. Dabrowski, ${ }^{84 a}$ F. Dachs,${ }^{36}$ T. Dado, ${ }^{47}$ S. Dahbi, ${ }^{33 e}$ T. Dai, ${ }^{106}$ C. Dallapiccola,${ }^{103}$ M. Dam, ${ }^{40}$ G. D'amen, ${ }^{29}$ V. D'Amico, ${ }^{75 a, 75 b}$ J. Damp, ${ }^{100}$ J. R. Dandoy, ${ }^{136}$ M. F. Daneri, ${ }^{30}$ M. Danninger, ${ }^{152}$ V. Dao,${ }^{36}$ G. Darbo, ${ }^{55 b}$ O. Dartsi, ${ }^{5}$ A. Dattagupta, ${ }^{131}$ T. Daubney, ${ }^{46}$ S. D’Auria ${ }^{69 \mathrm{a}, 69 \mathrm{~b}}$ C. David, ${ }^{168 \mathrm{~b}}$ T. Davidek, ${ }^{142}$ D. R. Davis,${ }^{49}$ I. Dawson, ${ }^{149}$ K. De,${ }^{8}$ R. De Asmundis ${ }^{70 \mathrm{a}}$ M. De Beurs, ${ }^{120}$ S. De Castro, ${ }^{23 b, 23 a}$ N. De Groot,${ }^{119}$ P. de Jong, ${ }^{120}$ H. De la Torre, ${ }^{107}$ A. De Maria, ${ }^{15 c}$ D. De Pedis, ${ }^{73 a}$ A. De Salvo, ${ }^{73 a}$ U. De Sanctis, ${ }^{74 a, 74 b}$ M. De Santis, ${ }^{74 a, 74 b}$ A. De Santo, ${ }^{156}$ J. B. De Vivie De Regie, ${ }^{65}$ D. V. Dedovich, ${ }^{80}$ A. M. Deiana, ${ }^{42}$ J. Del Peso, ${ }^{99}$ Y. Delabat Diaz, ${ }^{46}$ D. Delgove, ${ }^{65}$ F. Deliot,${ }^{144}$ C. M. Delitzsch, ${ }^{7}$ M. Della Pietra ${ }^{70 a, 70 b}$ D. Della Volpe,${ }^{54}$ A. Dell'Acqua, ${ }^{36}$ L. Dell'Asta ${ }^{74 a, 74 b}$ M. Delmastro, ${ }^{5}$ C. Delporte, ${ }^{65}$ P. A. Delsart,${ }^{58}$ S. Demers, ${ }^{183}$ M. Demichev, ${ }^{80}$ G. Demontigny, ${ }^{110}$ S. P. Denisov, ${ }^{123}$ L. D'Eramo, ${ }^{121}$ D. Derendarz, ${ }^{85}$ J. E. Derkaoui, ${ }^{35 d}$ F. Derue, ${ }^{135}$ P. Dervan, ${ }^{91}$ K. Desch, ${ }^{24}$ K. Dette,${ }^{167}$ C. Deutsch, ${ }^{24}$ M. R. Devesa ${ }^{30}$ P. O. Deviveiros,${ }^{36}$ F. A. Di Bello,${ }^{73 a, 73 b}$ A. Di Ciaccio, ${ }^{74 a}, 74 b$ L. Di Ciaccio, ${ }^{5}$ W. K. Di Clemente, ${ }^{136}$ C. Di Donato, ${ }^{70 a, 70 b}$ A. Di Girolamo, ${ }^{36}$ G. Di Gregorio,${ }^{72 a, 72 b}$ A. Di Luca, ${ }^{76 a, 76 b}$ B. Di Micco, ${ }^{75 a, 75 b}$ R. Di Nardo, ${ }^{75 a, 75 b}$ K. F. Di Petrillo,${ }^{59}$ R. Di Sipio, ${ }^{167}$ C. Diaconu, ${ }^{102}$ F. A. Dias, ${ }^{120}$ T. Dias Do Vale, ${ }^{139 a}$ M. A. Diaz, ${ }^{146 a}$ F. G. Diaz Capriles, ${ }^{24}$ J. Dickinson, ${ }^{18}$ M. Didenko, ${ }^{166}$ E. B. Diehl, ${ }^{106}$ J. Dietrich, ${ }^{19}$ S. Díez Cornell, ${ }^{46}$ C. Diez Pardos, ${ }^{151}$ A. Dimitrievska, ${ }^{18}$ W. Ding, ${ }^{15 b}$ J. Dingfelder, ${ }^{24}$ S. J. Dittmeier,${ }^{61 b}$ F. Dittus,${ }^{36}$ F. Djama, ${ }^{102}$ T. Djobava, ${ }^{159 b}$ J. I. Djuvsland, ${ }^{17}$ M. A. B. Do Vale,${ }^{147}$ M. Dobre, ${ }^{27 b}$ D. Dodsworth,${ }^{26}$ C. Doglioni, ${ }^{97}$ J. Dolejsi,${ }^{142}$ Z. Dolezal, ${ }^{142}$ M. Donadelli,${ }^{81 \mathrm{c}}$ B. Dong, ${ }^{60 \mathrm{c}}$ J. Donini, ${ }^{38}$ A. D'onofrio, ${ }^{15 c}$ M. D'Onofrio, ${ }^{91}$ J. Dopke, ${ }^{143}$ A. Doria, ${ }^{70 a}$ M. T. Dova, ${ }^{89}$ A. T. Doyle, ${ }^{57}$ E. Drechsler, ${ }^{152}$

E. Dreyer, ${ }^{152}$ T. Dreyer, ${ }^{53}$ A. S. Drobac, ${ }^{170}$ D. Du, ${ }^{60 b}$ T. A. du Pree, ${ }^{120}$ Y. Duan, ${ }^{60 d}$ F. Dubinin, ${ }^{111}$ M. Dubovsky, ${ }^{28 a}$ A. Dubreuil,${ }^{54}$ E. Duchovni, ${ }^{180}$ G. Duckeck, ${ }^{114}$ O. A. Ducu, ${ }^{36}$ D. Duda, ${ }^{115}$ A. Dudarev, ${ }^{36}$ A. C. Dudder,${ }^{100}$ E. M. Duffield, ${ }^{18}$ M. D’uffizi, ${ }^{101}$ L. Duflot, ${ }^{65}$ M. Dührssen, ${ }^{36}$ C. Dülsen, ${ }^{182}$ M. Dumancic,${ }^{180}$ A. E. Dumitriu, ${ }^{27 b}$ M. Dunford, ${ }^{61 a}$ S. Dungs,${ }^{47}$

A. Duperrin, ${ }^{102}$ H. Duran Yildiz, ${ }^{4 a}$ M. Düren ${ }^{56}$ A. Durglishvili, ${ }^{159 b}$ D. Duschinger ${ }^{48}$ B. Dutta, ${ }^{46}$ D. Duvnjak, ${ }^{1}$ G. I. Dyckes, ${ }^{136}$ M. Dyndal, ${ }^{36}$ S. Dysch, ${ }^{101}$ B. S. Dziedzic, ${ }^{85}$ M. G. Eggleston, ${ }^{49}$ T. Eifert, ${ }^{8}$ G. Eigen, ${ }^{17}$ K. Einsweiler, ${ }^{18}$ T. Ekelof, ${ }^{172}$ H. El Jarrari, ${ }^{35 e}$ V. Ellajosyula, ${ }^{172}$ M. Ellert, ${ }^{172}$ F. Ellinghaus, ${ }^{182}$ A. A. Elliot,${ }^{93}$ N. Ellis, ${ }^{36}$ J. Elmsheuser, ${ }^{29}$ M. Elsing ${ }^{36}$ D. Emeliyanov, ${ }^{143}$ A. Emerman, ${ }^{39}$ Y. Enari, ${ }^{163}$ M. B. Epland, ${ }^{49}$ J. Erdmann, ${ }^{47}$ A. Ereditato,${ }^{20}$ P. A. Erland, ${ }^{85}$ M. Errenst, ${ }^{182}$ M. Escalier, ${ }^{65}$ C. Escobar, ${ }^{174}$ O. Estrada Pastor, ${ }^{174}$ E. Etzion, ${ }^{161}$ G. E. Evans, ${ }^{139 a}$ H. Evans,${ }^{66}$ M. O. Evans, ${ }^{156}$ A. Ezhilov, ${ }^{137}$ F. Fabbri,${ }^{57}$ L. Fabbri, ${ }^{23 b, 23 a}$ V. Fabiani, ${ }^{119}$ G. Facini, ${ }^{178}$ R. M. Fakhrutdinov, ${ }^{123}$ S. Falciano, ${ }^{73 a}$ P. J. Falke, ${ }^{24}$ S. Falke, ${ }^{36}$ J. Faltova, ${ }^{142}$ Y. Fang, ${ }^{15 a}$ Y. Fang, ${ }^{15 a}$ G. Fanourakis, ${ }^{44}$ M. Fanti, ${ }^{69,69 b}$ M. Faraj, ${ }^{67 a, 67 c}$ A. Farbin, ${ }^{8}$ A. Farilla, ${ }^{75 a}$ E. M. Farina, ${ }^{71 \mathrm{a}, 71 \mathrm{~b}}$ T. Farooque, ${ }^{107}$ S. M. Farrington, ${ }^{50}$ P. Farthouat,${ }^{36}$ F. Fassi, ${ }^{35 \mathrm{e}}$ P. Fassnacht, ${ }^{36}$ D. Fassouliotis, ${ }^{9}$ M. Faucci Giannelli, ${ }^{50}$ W. J. Fawcett, ${ }^{32}$ L. Fayard, ${ }^{65}$ O. L. Fedin, ${ }^{137, \mathrm{~m}}$ W. Fedorko, ${ }^{175}$ A. Fehr, ${ }^{20}$ M. Feickert, ${ }^{173}$ L. Feligioni, ${ }^{102}$ A. Fell, ${ }^{149}$ C. Feng, ${ }^{60 b}$ M. Feng, ${ }^{49}$ M. J. Fenton, ${ }^{171}$ A. B. Fenyuk, ${ }^{123}$ S. W. Ferguson, ${ }^{43}$ J. Ferrando, ${ }^{46}$ 
A. Ferrari, ${ }^{172}$ P. Ferrari, ${ }^{120}$ R. Ferrari, ${ }^{71 \mathrm{a}}$ D. E. Ferreira de Lima, ${ }^{6 \mathrm{~b}}$ A. Ferrer, ${ }^{174}$ D. Ferrere, ${ }^{54}$ C. Ferretti, ${ }^{106}$ F. Fiedler, ${ }^{100}$ A. Filipčič,${ }^{92}$ F. Filthaut, ${ }^{119}$ K. D. Finelli, ${ }^{25}$ M. C. N. Fiolhais, ${ }^{139 a, 139 c, n}$ L. Fiorini, ${ }^{174}$ F. Fischer, ${ }^{114}$ J. Fischer, ${ }^{100}$ W. C. Fisher, ${ }^{107}$ T. Fitschen, ${ }^{21}$ I. Fleck, ${ }^{151}$ P. Fleischmann, ${ }^{106}$ T. Flick, ${ }^{182}$ B. M. Flierl, ${ }^{114}$ L. Flores, ${ }^{136}$ L. R. Flores Castillo, ${ }^{63 a}$ F. M. Follega, ${ }^{76 a, 76 b}$ N. Fomin, ${ }^{17}$ J. H. Foo, ${ }^{167}$ G. T. Forcolin, ${ }^{76 a, 76 b}$ B. C. Forland ${ }^{66}$ A. Formica, ${ }^{144}$ F. A. Förster, ${ }^{14}$

A. C. Forti, ${ }^{101}$ E. Fortin, ${ }^{102}$ M. G. Foti, ${ }^{134}$ D. Fournier, ${ }^{65}$ H. Fox, ${ }^{90}$ P. Francavilla, ${ }^{72 a, 72 b}$ S. Francescato, ${ }^{73 a, 73 b}$ M. Franchini, ${ }^{23 b, 23 a}$ S. Franchino, ${ }^{61 a}$ D. Francis,${ }^{36}$ L. Franco, ${ }^{5}$ L. Franconi, ${ }^{20}$ M. Franklin, ${ }^{59}$ G. Frattari, ${ }^{73 a, 73 b}$ A. N. Fray,${ }^{93}$ P. M. Freeman, ${ }^{21}$ B. Freund, ${ }^{110}$ W. S. Freund ${ }^{81 b}$ E. M. Freundlich,${ }^{47}$ D. C. Frizzell, ${ }^{128}$ D. Froidevaux ${ }^{36}$ J. A. Frost, ${ }^{134}$ M. Fujimoto, ${ }^{126}$ C. Fukunaga, ${ }^{164}$ E. Fullana Torregrosa, ${ }^{174}$ T. Fusayasu, ${ }^{116}$ J. Fuster, ${ }^{174}$ A. Gabrielli, ${ }^{23 b, 23 a}$ A. Gabrielli, ${ }^{36}$ S. Gadatsch ${ }^{54}$ P. Gadow, ${ }^{115}$ G. Gagliardi, ${ }^{55 b, 55 a}$ L. G. Gagnon, ${ }^{110}$ G. E. Gallardo, ${ }^{134}$ E. J. Gallas, ${ }^{134}$ B. J. Gallop, ${ }^{143}$ R. Gamboa Goni, ${ }^{93}$ K. K. Gan, ${ }^{127}$ S. Ganguly, ${ }^{180}$ J. Gao, ${ }^{60 a}$ Y. Gao, ${ }^{50}$ Y. S. Gao, ${ }^{31,0}$ F. M. Garay Walls, ${ }^{146 a}$ C. García, ${ }^{174}$ J. E. García Navarro, ${ }^{174}$ J. A. García Pascual, ${ }^{15 a}$ C. Garcia-Argos, ${ }^{52}$ M. Garcia-Sciveres, ${ }^{18}$ R. W. Gardner, ${ }^{37}$ N. Garelli, ${ }^{153}$ S. Gargiulo, ${ }^{52}$ C. A. Garner, ${ }^{167}$ V. Garonne, ${ }^{133}$ S. J. Gasiorowski, ${ }^{148}$ P. Gaspar ${ }^{81 b}$ A. Gaudiello, ${ }^{55 b, 55 a}$ G. Gaudio, ${ }^{71 a}$ P. Gauzzi, ${ }^{73 a, 73 b}$ I. L. Gavrilenko, ${ }^{111}$ A. Gavrilyuk, ${ }^{124}$ C. Gay, ${ }^{175}$ G. Gaycken, ${ }^{46}$ E. N. Gazis,${ }^{10}$ A. A. Geanta,${ }^{27 b}$ C. M. Gee, ${ }^{145}$ C. N. P. Gee, ${ }^{143}$ J. Geisen, ${ }^{97}$ M. Geisen, ${ }^{100}$ C. Gemme, ${ }^{55 b}$ M. H. Genest,${ }^{58}$ C. Geng, ${ }^{106}$ S. Gentile, ${ }^{73 a, 73 b}$ S. George,${ }^{94}$ T. Geralis, ${ }^{44}$ L. O. Gerlach, ${ }^{53}$ P. Gessinger-Befurt, ${ }^{100}$ G. Gessner, ${ }^{47}$ M. Ghasemi Bostanabad,${ }^{176}$ M. Ghneimat, ${ }^{151}$ A. Ghosh ${ }^{65}$ A. Ghosh ${ }^{78}$ B. Giacobbe ${ }^{23 b}$ S. Giagu, ${ }^{73 a, 73 b}$ N. Giangiacomi, ${ }^{167}$ P. Giannetti, ${ }^{72 a}$ A. Giannini $,{ }^{70 a}, 70 b$ G. Giannini, ${ }^{14}$ S. M. Gibson, ${ }^{94}$ M. Gignac, ${ }^{145}$ D. T. Gil ${ }^{84 b}$ B. J. Gilbert, ${ }^{39}$ D. Gillberg, ${ }^{34}$ G. Gilles, ${ }^{182}$ N. E. K. Gillwald, ${ }^{46}$ D. M. Gingrich, ${ }^{3, d}$ M. P. Giordani, ${ }^{67 a, 67 c}$ P. F. Giraud, ${ }^{144}$ G. Giugliarelli, ${ }^{67 a, 67 c}$ D. Giugni, ${ }^{69 a}$ F. Giuli, ${ }^{74 a, 74 b}$ S. Gkaitatzis, ${ }^{162}$ I. Gkialas, ${ }^{9, p}$ E. L. Gkougkousis, ${ }^{14}$ P. Gkountoumis, ${ }^{10}$ L. K. Gladilin, ${ }^{113}$ C. Glasman, ${ }^{99}$ J. Glatzer, ${ }^{14}$ P. C. F. Glaysher, ${ }^{46}$ A. Glazov, ${ }^{46}$ G. R. Gledhill, ${ }^{131}$ I. Gnesi, ${ }^{41 \mathrm{~b}, \mathrm{q}}$ M. Goblirsch-Kolb, ${ }^{26}$ D. Godin, ${ }^{110}$ S. Goldfarb, ${ }^{105}$ T. Golling, ${ }^{54}$ D. Golubkov, ${ }^{123}$ A. Gomes, ${ }^{139 a, 139 b}$ R. Goncalves Gama,${ }^{53}$ R. Gonçalo, ${ }^{139 a, 139 c}$ G. Gonella, ${ }^{131}$ L. Gonella, ${ }^{21}$ A. Gongadze ${ }^{80}$ F. Gonnella, ${ }^{21}$ J. L. Gonski, ${ }^{39}$ S. González de la Hoz, ${ }^{174}$ S. Gonzalez Fernandez, ${ }^{14}$ R. Gonzalez Lopez, ${ }^{91}$

C. Gonzalez Renteria, ${ }^{18}$ R. Gonzalez Suarez, ${ }^{172}$ S. Gonzalez-Sevilla, ${ }^{54}$ G. R. Gonzalvo Rodriguez, ${ }^{174}$ L. Goossens,${ }^{36}$ N. A. Gorasia, ${ }^{21}$ P. A. Gorbounov, ${ }^{124}$ H. A. Gordon, ${ }^{29}$ B. Gorini,${ }^{36}$ E. Gorini, ${ }^{68 a, 68 b}$ A. Gorišek,${ }^{92}$ A. T. Goshaw, ${ }^{49}$ M. I. Gostkin, ${ }^{80}$ C. A. Gottardo, ${ }^{119}$ M. Gouighri, ${ }^{35 b}$ A. G. Goussiou, ${ }^{148}$ N. Govender, ${ }^{33 c}$ C. Goy, ${ }^{5}$ I. Grabowska-Bold ${ }^{84 a}$ E. C. Graham, ${ }^{91}$ J. Gramling, ${ }^{171}$ E. Gramstad, ${ }^{133}$ S. Grancagnolo, ${ }^{19}$ M. Grandi, ${ }^{156}$ V. Gratchev, ${ }^{137}$ P. M. Gravila, ${ }^{27 f}$ F. G. Gravili ${ }^{68 a, 68 b}$ C. Gray, ${ }^{57}$ H. M. Gray, ${ }^{18}$ C. Grefe, ${ }^{24}$ K. Gregersen, ${ }^{97}$ I. M. Gregor, ${ }^{46}$ P. Grenier, ${ }^{153}$ K. Grevtsov,${ }^{46}$ C. Grieco,${ }^{14}$ N. A. Grieser, ${ }^{128}$ A. A. Grillo, ${ }^{145}$ K. Grimm, ${ }^{31, r}$ S. Grinstein, ${ }^{14, s}$ J.-F. Grivaz, ${ }^{65}$ S. Groh, ${ }^{100}$ E. Gross, ${ }^{180}$ J. Grosse-Knetter, ${ }^{53}$ Z. J. Grout, ${ }^{95}$ C. Grud, ${ }^{106}$ A. Grummer, ${ }^{118}$ J. C. Grundy, ${ }^{134}$ L. Guan, ${ }^{106}$ W. Guan, ${ }^{181}$ C. Gubbels,${ }^{175}$ J. Guenther, ${ }^{77}$ A. Guerguichon, ${ }^{65}$ J. G. R. Guerrero Rojas, ${ }^{174}$ F. Guescini, ${ }^{115}$ D. Guest, ${ }^{77}$ R. Gugel, ${ }^{100}$ A. Guida, ${ }^{46}$ T. Guillemin, ${ }^{5}$ S. Guindon, ${ }^{36}$ J. Guo, ${ }^{60 \mathrm{c}}$ W. Guo, ${ }^{106}$ Y. Guo, ${ }^{60 \mathrm{a}}$ Z. Guo, ${ }^{102}$ R. Gupta, ${ }^{46}$ S. Gurbuz, ${ }^{12 \mathrm{c}}$ G. Gustavino, ${ }^{128}$ M. Guth, ${ }^{52}$ P. Gutierrez, ${ }^{128}$ C. Gutschow,${ }^{95}$ C. Guyot, ${ }^{144}$ C. Gwenlan, ${ }^{134}$ C. B. Gwilliam, ${ }^{91}$ E. S. Haaland, ${ }^{133}$ A. Haas, ${ }^{125}$ C. Haber, ${ }^{18}$ H. K. Hadavand, ${ }^{8}$ A. Hadef,${ }^{100}$ M. Haleem, ${ }^{177}$ J. Haley, ${ }^{129}$ J. J. Hall, ${ }^{149}$ G. Halladjian, ${ }^{107}$ G. D. Hallewell, ${ }^{102}$ K. Hamano, ${ }^{176}$ H. Hamdaoui, ${ }^{35 \mathrm{e}}$ M. Hamer, ${ }^{24}$ G. N. Hamity,${ }^{50}$ K. Han ${ }^{60 \mathrm{a}}$ L. Han, ${ }^{15 \mathrm{c}}$ L. Han, ${ }^{60 \mathrm{a}}$ S. Han,${ }^{18}$ Y. F. Han,${ }^{167}$ K. Hanagaki, ${ }^{82, t}$ M. Hance, ${ }^{145}$ D. M. Handl, ${ }^{114}$ M. D. Hank,${ }^{37}$ R. Hankache, ${ }^{135}$ E. Hansen, ${ }^{97}$ J. B. Hansen, ${ }^{40}$ J. D. Hansen, ${ }^{40}$ M. C. Hansen, ${ }^{24}$ P. H. Hansen, ${ }^{40}$ E. C. Hanson, ${ }^{101}$ K. Hara, ${ }^{169}$ T. Harenberg, ${ }^{182}$ S. Harkusha, ${ }^{108}$ P. F. Harrison, ${ }^{178}$ N. M. Hartman, ${ }^{153}$ N. M. Hartmann, ${ }^{114}$ Y. Hasegawa,${ }^{150}$ A. Hasib,${ }^{50}$ S. Hassani,${ }^{144}$ S. Haug, ${ }^{20}$ R. Hauser, ${ }^{107}$ M. Havranek, ${ }^{141}$ C. M. Hawkes, ${ }^{21}$ R. J. Hawkings, ${ }^{36}$ S. Hayashida, ${ }^{117}$ D. Hayden, ${ }^{107}$ C. Hayes, ${ }^{106}$ R. L. Hayes, ${ }^{175}$ C. P. Hays,${ }^{134}$ J. M. Hays,${ }^{93}$ H. S. Hayward, ${ }^{91}$ S. J. Haywood, ${ }^{143}$ F. He, ${ }^{60 a}$ Y. He, ${ }^{165}$ M. P. Heath,${ }^{50}$ V. Hedberg, ${ }^{97}$ A. L. Heggelund, ${ }^{133}$ N. D. Hehir, ${ }^{93}$ C. Heidegger, ${ }^{52}$ K. K. Heidegger, ${ }^{52}$ W. D. Heidorn, ${ }^{79}$ J. Heilman, ${ }^{34}$ S. Heim, ${ }^{46}$ T. Heim, ${ }^{18}$ B. Heinemann, ${ }^{46, u}$

J. G. Heinlein, ${ }^{136}$ J. J. Heinrich, ${ }^{131}$ L. Heinrich,${ }^{36}$ J. Hejbal, ${ }^{140}$ L. Helary, ${ }^{46}$ A. Held, ${ }^{125}$ S. Hellesund, ${ }^{133}$ C. M. Helling, ${ }^{145}$ S. Hellman, ${ }^{45 a, 45 b}$ C. Helsens,${ }^{36}$ R. C. W. Henderson, ${ }^{90}$ L. Henkelmann, ${ }^{32}$ A. M. Henriques Correia, ${ }^{36}$ H. Herde, ${ }^{26}$ Y. Hernández Jiménez, ${ }^{33 e}$ H. Herr, ${ }^{100}$ M. G. Herrmann, ${ }^{114}$ T. Herrmann, ${ }^{48}$ G. Herten, ${ }^{52}$ R. Hertenberger, ${ }^{114}$ L. Hervas,${ }^{36}$ G. G. Hesketh, ${ }^{95}$ N. P. Hessey, ${ }^{168 a}$ H. Hibi, ${ }^{83}$ S. Higashino, ${ }^{82}$ E. Higón-Rodriguez, ${ }^{174}$ K. Hildebrand, ${ }^{37}$ J. C. Hill, ${ }^{32}$ K. K. Hill, ${ }^{29}$ K. H. Hiller, ${ }^{46}$ S. J. Hillier, ${ }^{21}$ M. Hils, ${ }^{48}$ I. Hinchliffe, ${ }^{18}$ F. Hinterkeuser, ${ }^{24}$ M. Hirose, ${ }^{132}$ S. Hirose, ${ }^{169}$ D. Hirschbuehl, ${ }^{182}$ B. Hiti, ${ }^{92}$ O. Hladik, ${ }^{140}$ J. Hobbs, ${ }^{155}$ R. Hobincu, ${ }^{27 \mathrm{e}}$ N. Hod, ${ }^{180}$ M. C. Hodgkinson, ${ }^{149}$ A. Hoecker, ${ }^{36}$ D. Hohn, ${ }^{52}$ D. Hohov, ${ }^{65}$ T. Holm, ${ }^{24}$ T. R. Holmes, ${ }^{37}$ M. Holzbock, ${ }^{115}$ L. B. A. H. Hommels, ${ }^{32}$ T. M. Hong, ${ }^{138}$ J. C. Honig, ${ }^{52}$ A. Hönle, ${ }^{115}$ B. H. Hooberman, ${ }^{173}$ W. H. Hopkins, ${ }^{6}$ Y. Horii, ${ }^{117}$ P. Horn, ${ }^{48}$ L. A. Horyn, ${ }^{37}$ S. Hou, ${ }^{158}$ A. Hoummada,${ }^{35 a}$ J. Howarth, ${ }^{57}$ J. Hoya, ${ }^{89}$ M. Hrabovsky, ${ }^{130}$ J. Hrivnac, ${ }^{65}$ A. Hrynevich, ${ }^{109}$ T. Hryn'ova, ${ }^{5}$ P. J. Hsu, ${ }^{64}$ S.-C. Hsu, ${ }^{148}$ Q. Hu, ${ }^{39}$ 
S. Hu, ${ }^{60 \mathrm{c}}$ Y. F. Hu, ${ }^{15 a, 15 d, v}$ D. P. Huang, ${ }^{95}$ X. Huang, ${ }^{15 c}$ Y. Huang,${ }^{60 a}$ Y. Huang, ${ }^{15 \mathrm{a}}$ Z. Hubacek, ${ }^{141}$ F. Hubaut, ${ }^{102}$ M. Huebner, ${ }^{24}$ F. Huegging, ${ }^{24}$ T. B. Huffman, ${ }^{134}$ M. Huhtinen, ${ }^{36}$ R. Hulsken, ${ }^{58}$ R. F. H. Hunter, ${ }^{34}$ N. Huseynov, ${ }^{80, w}$ J. Huston, ${ }^{107}$ J. Huth, ${ }^{59}$ R. Hyneman, ${ }^{153}$ S. Hyrych, ${ }^{28 a}$ G. Iacobucci, ${ }^{54}$ G. Iakovidis, ${ }^{29}$ I. Ibragimov, ${ }^{151}$ L. Iconomidou-Fayard ${ }^{65}$ P. Iengo, ${ }^{36}$ R. Ignazzi, ${ }^{40}$ R. Iguchi, ${ }^{163}$ T. Iizawa, ${ }^{54}$ Y. Ikegami,${ }^{82}$ M. Ikeno, ${ }^{82}$ N. Ilic,${ }^{119,167,1}$ F. Iltzsche, ${ }^{48}$ H. Imam, ${ }^{35 a}$ G. Introzzi,${ }^{71 a, 71 b}$ M. Iodice, ${ }^{75 a}$ K. Iordanidou, ${ }^{168 a}$ V. Ippolito, ${ }^{73 a, 73 b}$ M. F. Isacson, ${ }^{172}$ M. Ishino, ${ }^{163}$ W. Islam, ${ }^{129}$ C. Issever, ${ }^{19,46}$ S. Istin, ${ }^{160}$ J. M. Iturbe Ponce, ${ }^{63 a}$ R. Iuppa,${ }^{76 a, 76 b}$ A. Ivina, ${ }^{180}$ J. M. Izen, ${ }^{43}$ V. Izzo, ${ }^{70 a}$ P. Jacka, ${ }^{140}$ P. Jackson, ${ }^{1}$ R. M. Jacobs, ${ }^{46}$ B. P. Jaeger, ${ }^{152}$ V. Jain, ${ }^{2}$ G. Jäkel, ${ }^{182}$ K. B. Jakobi, ${ }^{100}$ K. Jakobs, ${ }^{52}$ T. Jakoubek, ${ }^{180}$ J. Jamieson, ${ }^{57}$ K. W. Janas, ${ }^{84 a}$ R. Jansky,${ }^{54}$ M. Janus, ${ }^{53}$ P. A. Janus, ${ }^{84 a}$ G. Jarlskog, ${ }^{97}$ A. E. Jaspan, ${ }^{91}$ N. Javadov,${ }^{80, w}$ T. Javůrek,${ }^{36}$ M. Javurkova, ${ }^{103}$ F. Jeanneau, ${ }^{144}$ L. Jeanty, ${ }^{131}$ J. Jejelava, ${ }^{159 a}$ P. Jenni,${ }^{52, x}$ N. Jeong, ${ }^{46}$ S. Jézéquel,,${ }^{5}$ J. Jia, ${ }^{155}$ Z. Jia, ${ }^{15 c}$ H. Jiang, ${ }^{79}$ Y. Jiang, ${ }^{60 a}$ Z. Jiang, ${ }^{153}$ S. Jiggins,${ }^{52}$ F. A. Jimenez Morales, ${ }^{38}$ J. Jimenez Pena, ${ }^{115}$ S. Jin, ${ }^{15 \mathrm{c}}$ A. Jinaru, ${ }^{27 \mathrm{~b}}$ O. Jinnouchi, ${ }^{165} \mathrm{H}$. Jivan, ${ }^{33 \mathrm{e}}$ P. Johansson, ${ }^{149}$ K. A. Johns, ${ }^{7}$ C. A. Johnson, ${ }^{66}$ E. Jones, ${ }^{178}$ R. W. L. Jones, ${ }^{90}$ S. D. Jones, ${ }^{156}$ T. J. Jones, ${ }^{91}$ J. Jovicevic, ${ }^{36}$ X. Ju, ${ }^{18}$ J. J. Junggeburth, ${ }^{115}$ A. Juste Rozas, ${ }^{14, \mathrm{~s}}$ A. Kaczmarska, ${ }^{85}$ M. Kado, ${ }^{73 a, 73 b}$ H. Kagan, ${ }^{127}$ M. Kagan, ${ }^{153}$ A. Kahn, ${ }^{39}$ C. Kahra, ${ }^{100}$ T. Kaji, ${ }^{179}$ E. Kajomovitz, ${ }^{160}$ C. W. Kalderon, ${ }^{29}$ A. Kaluza, ${ }^{100}$ A. Kamenshchikov, ${ }^{123}$ M. Kaneda, ${ }^{163}$ N. J. Kang, ${ }^{145}$ S. Kang, ${ }^{79}$ Y. Kano, ${ }^{117}$ J. Kanzaki, ${ }^{82}$ L. S. Kaplan, ${ }^{181}$ D. Kar, ${ }^{33 \mathrm{e}}$ K. Karava, ${ }^{134}$ M. J. Kareem, ${ }^{168 \mathrm{~b}}$ I. Karkanias, ${ }^{162}$ S. N. Karpov, ${ }^{80}$ Z. M. Karpova,${ }^{80}$ V. Kartvelishvili, ${ }^{90}$ A. N. Karyukhin, ${ }^{123}$ E. Kasimi, ${ }^{162}$ A. Kastanas,,${ }^{45 a, 45 b}$ C. Kato, ${ }^{60 d}$ J. Katzy, ${ }^{46}$ K. Kawade, ${ }^{150}$ K. Kawagoe, ${ }^{88}$ T. Kawaguchi, ${ }^{117}$ T. Kawamoto, ${ }^{144}$ G. Kawamura, ${ }^{53}$ E. F. Kay, ${ }^{176}$ F. I. Kaya, ${ }^{170}$ S. Kazakos, ${ }^{14}$ V. F. Kazanin, ${ }^{122 b, 122 a}$ J. M. Keaveney, ${ }^{33 a}$ R. Keeler, ${ }^{176}$ J. S. Keller, ${ }^{34}$ E. Kellermann, ${ }^{97}$ D. Kelsey, ${ }^{156}$ J. J. Kempster, ${ }^{21}$ J. Kendrick, ${ }^{21}$ K. E. Kennedy, ${ }^{39}$ O. Kepka, ${ }^{140}$ S. Kersten, ${ }^{182}$ B. P. Kerševan, ${ }^{92}$ S. Ketabchi Haghighat, ${ }^{167}$ F. Khalil-Zada, ${ }^{13}$ M. Khandoga ${ }^{144}$ A. Khanov, ${ }^{129}$ A. G. Kharlamov, ${ }^{122 b, 122 a}$ T. Kharlamova, ${ }^{122 b, 122 a}$ E. E. Khoda, ${ }^{175}$ T. J. Khoo, ${ }^{77}$ G. Khoriauli, ${ }^{177}$ E. Khramov, ${ }^{80}$ J. Khubua, ${ }^{159 b}$ S. Kido, ${ }^{83}$ M. Kiehn, ${ }^{36}$ E. Kim, ${ }^{165}$ Y. K. Kim, ${ }^{37}$ N. Kimura, ${ }^{95}$ A. Kirchhoff, ${ }^{53}$ D. Kirchmeier, ${ }^{48}$ J. Kirk, ${ }^{143}$ A. E. Kiryunin, ${ }^{115}$ T. Kishimoto, ${ }^{163}$ D. P. Kisliuk, ${ }^{167}$ V. Kitali, ${ }^{46}$ C. Kitsaki, ${ }^{10}$ O. Kivernyk, ${ }^{24}$ T. Klapdor-Kleingrothaus, ${ }^{52}$ M. Klassen, ${ }^{61 a}$ C. Klein, ${ }^{34}$ M. H. Klein, ${ }^{106}$ M. Klein, ${ }^{91}$ U. Klein, ${ }^{91}$ K. Kleinknecht, ${ }^{100}$ P. Klimek,${ }^{36}$ A. Klimentov, ${ }^{29}$ F. Klimpel, ${ }^{36}$ T. Klingl, ${ }^{24}$ T. Klioutchnikova, ${ }^{36}$ F. F. Klitzner, ${ }^{114}$ P. Kluit, ${ }^{120}$ S. Kluth, ${ }^{115}$ E. Kneringer,${ }^{77}$ E. B. F. G. Knoops, ${ }^{102}$ A. Knue, ${ }^{52}$ D. Kobayashi, ${ }^{88}$ M. Kobel,${ }^{48}$ M. Kocian, ${ }^{153}$ T. Kodama, ${ }^{163}$ P. Kodys, ${ }^{142}$ D. M. Koeck, ${ }^{156}$ P. T. Koenig, ${ }^{24}$ T. Koffas, ${ }^{34}$ N. M. Köhler, ${ }^{36}$ M. Kolb, ${ }^{144}$ I. Koletsou, ${ }^{5}$ T. Komarek, ${ }^{130}$ T. Kondo, ${ }^{82}$ K. Köneke, ${ }^{52}$ A. X. Y. Kong, ${ }^{1}$ A. C. König, ${ }^{119}$ T. Kono, ${ }^{126}$ V. Konstantinides, ${ }^{95}$ N. Konstantinidis, ${ }^{95}$ B. Konya, ${ }^{97}$ R. Kopeliansky, ${ }^{66}$ S. Koperny, ${ }^{84 a}$ K. Korcyl, ${ }^{85}$ K. Kordas, ${ }^{162}$ G. Koren, ${ }^{161}$ A. Korn, ${ }^{95}$ I. Korolkov, ${ }^{14}$ E. V. Korolkova, ${ }^{149}$ N. Korotkova, ${ }^{113}$ O. Kortner, ${ }^{115}$ S. Kortner, ${ }^{115}$ V. V. Kostyukhin, ${ }^{149,166}$ A. Kotsokechagia,${ }^{65}$ A. Kotwal, ${ }^{49}$ A. Koulouris, ${ }^{10}$ A. Kourkoumeli-Charalampidi, ${ }^{71 a, 71 b}$ C. Kourkoumelis, ${ }^{9}$ E. Kourlitis, ${ }^{6}$ V. Kouskoura, ${ }^{29}$ R. Kowalewski, ${ }^{176}$ W. Kozanecki, ${ }^{101}$ A. S. Kozhin, ${ }^{123}$ V. A. Kramarenko, ${ }^{113}$ G. Kramberger, ${ }^{92}$ D. Krasnopevtsev, ${ }^{60 a}$ M. W. Krasny, ${ }^{135}$ A. Krasznahorkay, ${ }^{36}$ D. Krauss, ${ }^{115}$ J. A. Kremer, ${ }^{100}$ J. Kretzschmar, ${ }^{91}$ K. Kreul, ${ }^{19}$ P. Krieger, ${ }^{167}$ F. Krieter,${ }^{114}$ S. Krishnamurthy, ${ }_{103}$ A. Krishnan, ${ }^{61 b}$ M. Krivos, ${ }^{142}$ K. Krizka, ${ }^{18}$ K. Kroeninger ${ }^{47}$ H. Kroha, ${ }^{115}$ J. Kroll ${ }^{140}$ J. Kroll, ${ }^{136}$ K. S. Krowpman, ${ }^{107}$ U. Kruchonak, ${ }^{80}$ H. Krüger ${ }^{24}$ N. Krumnack, ${ }^{79}$ M. C. Kruse, ${ }^{49}$ J. A. Krzysiak, ${ }^{85}$ A. Kubota, ${ }^{165}$ O. Kuchinskaia, ${ }^{166}$ S. Kuday, ${ }^{4 b}$ D. Kuechler, ${ }^{46}$ J. T. Kuechler ${ }^{46}$ S. Kuehn, ${ }^{36}$ T. Kuhl, ${ }^{46}$ V. Kukhtin, ${ }^{80}$ Y. Kulchitsky, ${ }^{108, y}$ S. Kuleshov, ${ }^{146 b}$ Y. P. Kulinich, ${ }^{173}$ M. Kuna ${ }^{58}$ A. Kupco, ${ }^{140}$ T. Kupfer, ${ }^{47}$ O. Kuprash, ${ }^{52}$ H. Kurashige, ${ }^{83}$ L. L. Kurchaninov, ${ }^{168 a}$ Y. A. Kurochkin, ${ }^{108}$ A. Kurova, ${ }^{112}$ M. G. Kurth, ${ }^{15 a, 15 d}$ E. S. Kuwertz, ${ }^{36}$ M. Kuze, ${ }^{165}$ A. K. Kvam, ${ }^{148}$ J. Kvita, ${ }^{130}$ T. Kwan, ${ }^{104}$ C. Lacasta, ${ }^{174}$ F. Lacava, ${ }^{73 a, 73 b}$ D. P. J. Lack, ${ }^{101}$ H. Lacker, ${ }^{19}$ D. Lacour, ${ }^{135}$ E. Ladygin, ${ }^{80}$ R. Lafaye, ${ }^{5}$ B. Laforge, ${ }^{135}$ T. Lagouri, ${ }^{146 c}$ S. Lai, ${ }^{53}$ I. K. Lakomiec, ${ }^{84 a}$ J. E. Lambert, ${ }^{128}$ S. Lammers, ${ }^{66}$ W. Lampl, ${ }^{7}$ C. Lampoudis, ${ }^{162}$ E. Lançon, ${ }^{29}$ U. Landgraf, ${ }^{52}$ M. P. J. Landon, ${ }^{93}$ V. S. Lang, ${ }^{52}$ J. C. Lange, ${ }^{53}$ R. J. Langenberg, ${ }^{103}$ A. J. Lankford, ${ }^{171}$ F. Lanni, ${ }^{29}$ K. Lantzsch, ${ }^{24}$ A. Lanza, ${ }^{71 a}$ A. Lapertosa, ${ }^{55,55 a}$ J. F. Laporte, ${ }^{144}$ T. Lari, ${ }^{69 a}$ F. Lasagni Manghi, ${ }^{23 b, 23 a}$ M. Lassnig, ${ }^{36}$ V. Latonova, ${ }^{140}$ T. S. Lau, ${ }^{63 a}$ A. Laudrain, ${ }^{100}$ A. Laurier, ${ }^{34}$ M. Lavorgna, ${ }^{70 a, 70 b}$ S. D. Lawlor ${ }^{94}$ M. Lazzaroni, ${ }^{69 a, 69 b}$ B. Le, ${ }^{101}$ E. Le Guirriec, ${ }^{102}$ A. Lebedev ${ }^{79}$ M. LeBlanc, ${ }^{7}$ T. LeCompte, ${ }^{6}$ F. Ledroit-Guillon, ${ }^{58}$ A. C. A. Lee,${ }^{95}$ C. A. Lee, ${ }^{29}$ G. R. Lee,${ }^{17}$ L. Lee, ${ }^{59}$ S. C. Lee, ${ }^{158}$ S. Lee,${ }^{79}$ B. Lefebvre, ${ }^{168 a}$ H. P. Lefebvre, ${ }^{94}$ M. Lefebvre, ${ }^{176}$ C. Leggett, ${ }^{18}$ K. Lehmann, ${ }^{152}$ N. Lehmann, ${ }^{20}$ G. Lehmann Miotto, ${ }^{36}$ W. A. Leight, ${ }^{46}$ A. Leisos, ${ }^{162, z}$ M. A. L. Leite, ${ }^{81 c}$ C. E. Leitgeb, ${ }^{114}$ R. Leitner, ${ }^{142}$ K. J. C. Leney, ${ }^{42}$ T. Lenz,${ }^{24}$ S. Leone, ${ }^{72 a}$ C. Leonidopoulos, ${ }^{50}$ A. Leopold, ${ }^{135}$ C. Leroy, ${ }^{110}$ R. Les, ${ }^{107}$ C. G. Lester, ${ }^{32}$ M. Levchenko, ${ }^{137}$ J. Levêque, ${ }^{5}$ D. Levin, ${ }^{106}$ L. J. Levinson, ${ }^{180}$ D. J. Lewis, ${ }^{21}$ B. Li ${ }^{15 b}$ B. Li $,{ }^{106} \mathrm{C}-\mathrm{Q} . \mathrm{Li},{ }^{60 \mathrm{c}, 60 \mathrm{~d}} \mathrm{~F} . \mathrm{Li},{ }^{60 \mathrm{c}} \mathrm{H} . \mathrm{Li},{ }^{60 \mathrm{a}} \mathrm{H} . \mathrm{Li},{ }^{60 \mathrm{~b}} \mathrm{~J} . \mathrm{Li},{ }^{60 \mathrm{c}}$

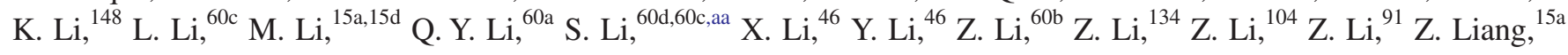


M. Liberatore, ${ }^{46}$ B. Liberti, ${ }^{74 a}$ K. Lie ${ }^{63 c}$ S. Lim,${ }^{29}$ C. Y. Lin, ${ }^{32}$ K. Lin, ${ }^{107}$ R. A. Linck ${ }^{66}$ R. E. Lindley, ${ }^{7}$ J. H. Lindon, ${ }^{21}$ A. Linss, ${ }^{46}$ A. L. Lionti,${ }^{54}$ E. Lipeles, ${ }^{136}$ A. Lipniacka, ${ }^{17}$ T. M. Liss, ${ }^{173, b b}$ A. Lister,${ }^{175}$ J. D. Little,${ }^{8}$ B. Liu, ${ }^{79}$ B. X. Liu, ${ }^{152}$ H. B. Liu, ${ }^{29}$ J. B. Liu, ${ }^{60 a}$ J. K. K. Liu, ${ }^{37}$ K. Liu, ${ }^{60 d}$ M. Liu, ${ }^{60 a}$ M. Y. Liu, ${ }^{60 a}$ P. Liu, ${ }^{15 a}$ X. Liu, ${ }^{60 a}$ Y. Liu, ${ }^{46}$ Y. Liu, ${ }^{15 a, 15 d}$ Y. L. Liu, ${ }^{106}$ Y. W. Liu, ${ }^{60 a}$ M. Livan, ${ }^{71 a, 71 b}$ A. Lleres, ${ }^{58}$ J. Llorente Merino, ${ }^{152}$ S. L. Lloyd, ${ }^{93}$ C. Y. Lo, ${ }^{63 b}$

E. M. Lobodzinska, ${ }^{46}$ P. Loch, ${ }^{7}$ S. Loffredo, ${ }^{74 a, 74 b}$ T. Lohse, ${ }^{19}$ K. Lohwasser, ${ }^{149}$ M. Lokajicek, ${ }^{140}$ J. D. Long, ${ }^{173}$ R. E. Long,${ }^{90}$ I. Longarini, ${ }^{73 a, 73 b}$ L. Longo, ${ }^{36}$ I. Lopez Paz, ${ }^{101}$ A. Lopez Solis, ${ }^{149}$ J. Lorenz, ${ }^{114}$ N. Lorenzo Martinez, ${ }^{5}$ A. M. Lory, ${ }^{114}$ A. Lösle ${ }^{52}$ X. Lou, ${ }^{45 a, 45 b}$ X. Lou, ${ }^{15 a}$ A. Lounis, ${ }^{65}$ J. Love, ${ }^{6}$ P. A. Love, ${ }^{90}$ J. J. Lozano Bahilo, ${ }^{174}$ M. Lu, ${ }^{60 a}$ Y. J. Lu, ${ }^{64}$ H. J. Lubatti, ${ }^{148}$ C. Luci, ${ }^{73 a, 73 b}$ F. L. Lucio Alves, ${ }^{15 c}$ A. Lucotte, ${ }^{58}$ F. Luehring, ${ }^{66}$ I. Luise, ${ }^{155}$ L. Luminari, ${ }^{73 a}$ B. Lund-Jensen, ${ }^{154}$ N. A. Luongo, ${ }^{131}$ M. S. Lutz, ${ }^{161}$ D. Lynn, ${ }^{29}$ H. Lyons, ${ }^{91}$ R. Lysak, ${ }^{140}$ E. Lytken, ${ }^{97}$ F. Lyu, ${ }^{15 a}$ V. Lyubushkin, ${ }^{80}$ T. Lyubushkina, ${ }^{80}$ H. Ma ${ }^{29}$ L. L. Ma, ${ }^{60 b}$ Y. Ma, ${ }^{95}$ D. M. Mac Donell, ${ }^{176}$ G. Maccarrone, ${ }^{51}$ C. M. Macdonald, ${ }^{149}$ J. C. MacDonald, ${ }^{149}$ J. Machado Miguens, ${ }^{136}$ R. Madar, ${ }^{38}$ W. F. Mader ${ }^{48}$

M. Madugoda Ralalage Don, ${ }^{129}$ N. Madysa, ${ }^{48}$ J. Maeda, ${ }^{83}$ T. Maeno, ${ }^{29}$ M. Maerker ${ }^{48}$ V. Magerl,${ }^{52}$ N. Magini, ${ }^{79}$ J. Magro, ${ }^{67 a, 67 c, c c}$ D. J. Mahon, ${ }^{39}$ C. Maidantchik, ${ }^{81 b}$ A. Maio, ${ }^{139 a, 139 b, 139 d}$ K. Maj, ${ }^{84 a}$ O. Majersky, ${ }^{28 a}$ S. Majewski, ${ }^{131}$ Y. Makida ${ }^{82}$ N. Makovec, ${ }^{65}$ B. Malaescu, ${ }^{135}$ Pa. Malecki, ${ }^{85}$ V. P. Maleev,${ }^{137}$ F. Malek,${ }^{58}$ D. Malito, ${ }^{41 b, 41 a}$ U. Mallik, ${ }^{78}$ C. Malone ${ }^{32}$ S. Maltezos, ${ }^{10}$ S. Malyukov ${ }^{80}$ J. Mamuzic, ${ }^{174}$ G. Mancini,${ }^{51}$ J. P. Mandalia, ${ }^{93}$ I. Mandić, ${ }^{92}$ L. Manhaes de Andrade Filho, ${ }^{81 a}$ I. M. Maniatis, ${ }^{162}$ J. Manjarres Ramos, ${ }^{48}$ K. H. Mankinen, ${ }^{97}$ A. Mann, ${ }^{114}$ A. Manousos, ${ }^{77}$ B. Mansoulie, ${ }^{144}$ I. Manthos, ${ }^{162}$ S. Manzoni, ${ }^{120}$ A. Marantis, ${ }^{162}$ G. Marceca, ${ }^{30}$ L. Marchese,${ }^{134}$ G. Marchiori, ${ }^{135}$ M. Marcisovsky, ${ }^{140}$ L. Marcoccia,${ }^{74 a, 74 b}$ C. Marcon,${ }^{97}$ M. Marjanovic, ${ }^{128}$ Z. Marshall, ${ }^{18}$ M. U. F. Martensson, ${ }^{172}$ S. Marti-Garcia, ${ }^{174}$ C. B. Martin, ${ }^{127}$ T. A. Martin, ${ }^{178}$ V. J. Martin, ${ }^{50}$ B. Martin dit Latour, ${ }^{17}$ L. Martinelli, ${ }^{75 a, 75 b}$ M. Martinez, ${ }^{14, \mathrm{~s}}$ P. Martinez Agullo, ${ }^{174}$ V. I. Martinez Outschoorn, ${ }^{103}$ S. Martin-Haugh, ${ }^{143}$ V. S. Martoiu, ${ }^{27 b}$ A. C. Martyniuk, ${ }^{95}$ A. Marzin, ${ }^{36}$ S. R. Maschek, ${ }^{115}$ L. Masetti, ${ }^{100}$ T. Mashimo, ${ }^{163}$ R. Mashinistov, ${ }^{111}$ J. Masik, ${ }^{101}$ A. L. Maslennikov, ${ }^{122 b, 122 a}$ L. Massa, ${ }^{23 b, 23 a}$ P. Massarotti, ${ }^{70 a, 70 b}$ P. Mastrandrea, ${ }^{72 a, 72 b}$ A. Mastroberardino, ${ }^{41 b, 41 a}$ T. Masubuchi, ${ }^{163}$ D. Matakias, ${ }^{29}$ A. Matic, ${ }^{114}$ N. Matsuzawa, ${ }^{163}$ P. Mättig,${ }^{24}$ J. Maurer, ${ }^{27 b}$ B. Maček,${ }^{92}$ D. A. Maximov, ${ }^{122 b, 122 a}$ R. Mazini, ${ }^{158}$ I. Maznas, ${ }^{162}$ S. M. Mazza, ${ }^{145}$ J. P. Mc Gowan, ${ }^{104}$ S. P. Mc Kee, ${ }^{106}$ T. G. McCarthy, ${ }^{115}$ W. P. McCormack, ${ }^{18}$ E. F. McDonald, ${ }^{105}$ A. E. McDougall, ${ }^{120}$ J. A. Mcfayden, ${ }^{18}$ G. Mchedlidze,${ }^{159 b}$ M. A. McKay, ${ }^{42}$ K. D. McLean, ${ }^{176}$ S. J. McMahon, ${ }^{143}$ P. C. McNamara, ${ }^{105}$ C. J. McNicol, ${ }^{178}$ R. A. McPherson, ${ }^{176,1}$ J. E. Mdhluli, ${ }^{33 e}$ Z. A. Meadows, ${ }^{103}$ S. Meehan, ${ }^{36}$ T. Megy, ${ }^{38}$ S. Mehlhase, ${ }^{114}$ A. Mehta,${ }^{91}$ B. Meirose, ${ }^{43}$ D. Melini, ${ }^{160}$ B. R. Mellado Garcia, ${ }^{33 e}$ J. D. Mellenthin, ${ }^{53}$ M. Melo, ${ }^{28 a}$ F. Meloni, ${ }^{46}$ A. Melzer, ${ }^{24}$ E. D. Mendes Gouveia, ${ }^{139 a, 139 e}$ A. M. Mendes Jacques Da Costa, ${ }^{21}$ H. Y. Meng, ${ }^{167}$ L. Meng, ${ }^{36}$ X. T. Meng, ${ }^{106}$ S. Menke, ${ }^{115}$ E. Meoni, ${ }^{41 b, 41 a}$ S. Mergelmeyer, ${ }^{19}$ S. A. M. Merkt, ${ }^{138}$ C. Merlassino, ${ }^{134}$ P. Mermod,${ }^{54}$ L. Merola,${ }^{70 a, 70 b}$ C. Meroni, ${ }^{69 a}$ G. Merz, ${ }^{106}$ O. Meshkov, ${ }^{113,111}$ J. K. R. Meshreki, ${ }^{151}$ J. Metcalfe, ${ }^{6}$ A. S. Mete, ${ }^{6}$ C. Meyer, ${ }^{66}$ J-P. Meyer, ${ }^{144}$ M. Michetti, ${ }^{19}$ R. P. Middleton, ${ }^{143}$ L. Mijović, ${ }^{50}$ G. Mikenberg, ${ }^{180}$ M. Mikestikova, ${ }^{140}$ M. Mikuž, ${ }^{92}$ H. Mildner, ${ }^{149}$ A. Milic, ${ }^{167}$ C. D. Milke, ${ }^{42}$ D. W. Miller, ${ }^{37}$ L. S. Miller, ${ }^{34}$ A. Milov, ${ }^{180}$ D. A. Milstead ${ }^{45 a, 45 b}$ A. A. Minaenko, ${ }^{123}$ I. A. Minashvili, ${ }^{159 b}$ L. Mince, ${ }^{57}$ A. I. Mincer, ${ }^{125}$ B. Mindur,${ }^{84 a}$ M. Mineev, ${ }^{80}$ Y. Minegishi, ${ }^{163}$ Y. Mino, ${ }^{86}$ L. M. Mir, ${ }^{14}$ M. Mironova, ${ }^{134}$ T. Mitani, ${ }^{179}$ J. Mitrevski, ${ }^{114}$ V. A. Mitsou, ${ }^{174}$ M. Mittal, ${ }^{60 c}$ O. Miu, ${ }^{167}$ A. Miucci, ${ }^{20}$ P. S. Miyagawa, ${ }^{93}$ A. Mizukami, ${ }^{82}$ J. U. Mjörnmark, ${ }^{97}$ T. Mkrtchyan, ${ }^{61 a}$ M. Mlynarikova, ${ }^{121}$ T. Moa, ${ }^{45 a, 45 b}$ S. Mobius,${ }^{53}$ K. Mochizuki, ${ }^{110}$ P. Moder, ${ }^{46}$ P. Mogg, ${ }^{114}$ S. Mohapatra, ${ }^{39}$ R. Moles-Valls, ${ }^{24}$ K. Mönig, ${ }^{46}$ E. Monnier, ${ }^{102}$ A. Montalbano, ${ }^{152}$ J. Montejo Berlingen, ${ }^{36}$ M. Montella, ${ }^{95}$ F. Monticelli, ${ }^{89}$ S. Monzani, ${ }^{69 a}$ N. Morange,${ }^{65}$ A. L. Moreira De Carvalho, ${ }^{139 a}$ D. Moreno, ${ }^{22 a}$ M. Moreno Llácer, ${ }^{174}$ C. Moreno Martinez, ${ }^{14}$ P. Morettini, ${ }^{55 b}$ M. Morgenstern, ${ }^{160}$ S. Morgenstern, ${ }^{48}$ D. Mori, ${ }^{152}$ M. Morii, ${ }^{59}$ M. Morinaga, ${ }^{179}$ V. Morisbak, ${ }^{133}$ A. K. Morley, ${ }^{36}$ G. Mornacchi, ${ }^{36}$ A. P. Morris, ${ }^{95}$ L. Morvaj, ${ }^{36}$ P. Moschovakos, ${ }^{36}$ B. Moser, ${ }^{120}$ M. Mosidze, ${ }^{159 b}$ T. Moskalets, ${ }^{144}$ P. Moskvitina, ${ }^{119}$ J. Moss, ${ }^{31, d d}$ E. J. W. Moyse, ${ }^{103}$ S. Muanza,${ }^{102}$ J. Mueller, ${ }^{138}$ R. S. P. Mueller, ${ }^{114}$ D. Muenstermann, ${ }^{90}$ G. A. Mullier, ${ }^{97}$ D. P. Mungo, ${ }^{69 a, 69 b}$ J. L. Munoz Martinez, ${ }^{14}$ F. J. Munoz Sanchez, ${ }^{101}$ P. Murin, ${ }^{28 b}$ W. J. Murray, ${ }^{17,143}$ A. Murrone,${ }^{6 a, 69 b}$ J. M. Muse, ${ }^{128}$ M. Muškinja, ${ }^{18}$ C. Mwewa, ${ }^{33 a}$ A. G. Myagkov, ${ }^{123, i}$ A. A. Myers, ${ }^{138}$ G. Myers, ${ }^{66}$ J. Myers, ${ }^{131}$ M. Myska, ${ }^{141}$ B. P. Nachman, ${ }^{18}$ O. Nackenhorst, ${ }^{47}$ A. Nag Nag, ${ }^{48}$ K. Nagai, ${ }^{134}$ K. Nagano, ${ }^{82}$ Y. Nagasaka, ${ }^{62}$ J. L. Nagle, ${ }^{29}$ E. Nagy, ${ }^{102}$ A. M. Nairz, ${ }^{36}$ Y. Nakahama, ${ }^{117}$ K. Nakamura, ${ }^{82}$ T. Nakamura, ${ }^{163}$ H. Nanjo, ${ }^{132}$ F. Napolitano, ${ }^{61 a}$ R. F. Naranjo Garcia, ${ }^{46}$ R. Narayan, ${ }^{42}$ I. Naryshkin, ${ }^{137}$ M. Naseri, ${ }^{34}$ T. Naumann, ${ }^{46}$ G. Navarro, ${ }^{22 a}$ P. Y. Nechaeva, ${ }^{111}$ F. Nechansky, ${ }^{46}$ T. J. Neep, ${ }^{21}$ A. Negri, ${ }^{71 a, 71 b}$ M. Negrini, ${ }^{23 b}$ C. Nellist, ${ }^{119}$ C. Nelson, ${ }^{104}$ M. E. Nelson, ${ }^{45 a, 45 b}$ S. Nemecek, ${ }^{140}$ M. Nessi,,${ }^{36, e e}$ M. S. Neubauer, ${ }^{173}$ F. Neuhaus, ${ }^{100}$ 
M. Neumann, ${ }^{182}$ R. Newhouse, ${ }^{175}$ P. R. Newman, ${ }^{21}$ C. W. Ng, ${ }^{138}$ Y. S. Ng, ${ }^{19}$ Y. W. Y. Ng, ${ }^{171}$ B. Ngair, ${ }^{35 e}$ H. D. N. Nguyen, ${ }^{102}$ T. Nguyen Manh, ${ }^{110}$ E. Nibigira, ${ }^{38}$ R. B. Nickerson, ${ }^{134}$ R. Nicolaidou, ${ }^{144}$ D. S. Nielsen, ${ }^{40}$ J. Nielsen, ${ }^{145}$ M. Niemeyer, ${ }^{53}$ N. Nikiforou, ${ }^{11}$ V. Nikolaenko, ${ }^{123, i}$ I. Nikolic-Audit, ${ }^{135}$ K. Nikolopoulos, ${ }^{21}$ P. Nilsson, ${ }^{29}$ H. R. Nindhito, ${ }^{54}$ A. Nisati, ${ }^{73 a}$ N. Nishu, ${ }^{60 \mathrm{c}}$ R. Nisius, ${ }^{115}$ I. Nitsche,${ }^{47}$ T. Nitta, ${ }^{179}$ T. Nobe,${ }^{163}$ D. L. Noel,${ }^{32}$ Y. Noguchi ${ }^{86}$ I. Nomidis, ${ }^{135}$ M. A. Nomura, ${ }^{29}$ M. Nordberg, ${ }^{36}$ J. Novak, ${ }^{92}$ T. Novak, ${ }^{92}$ O. Novgorodova, ${ }^{48}$ R. Novotny, ${ }^{118}$ L. Nozka, ${ }^{130}$ K. Ntekas, ${ }^{171}$ E. Nurse, ${ }^{95}$

F. G. Oakham, ${ }^{34, \mathrm{~d}}$ J. Ocariz, ${ }^{135}$ A. Ochi, ${ }^{83}$ I. Ochoa, ${ }^{139 a}$ J. P. Ochoa-Ricoux, ${ }^{146 a}$ K. O'Connor, ${ }^{26}$ S. Oda, ${ }^{88}$ S. Odaka, ${ }^{82}$ S. Oerdek, ${ }^{53}$ A. Ogrodnik, ${ }^{84 a}$ A. Oh, ${ }^{101}$ C. C. Ohm, ${ }^{154}$ H. Oide, ${ }^{165}$ R. Oishi, ${ }^{163}$ M. L. Ojeda, ${ }^{167}$ H. Okawa, ${ }^{169}$ Y. Okazaki ${ }^{86}$ M. W. O'Keefe, ${ }^{91}$ Y. Okumura, ${ }^{163}$ A. Olariu, ${ }^{27 b}$ L. F. Oleiro Seabra, ${ }^{139 a}$ S. A. Olivares Pino, ${ }^{146 a}$ D. Oliveira Damazio, ${ }^{29}$ J. L. Oliver, ${ }^{1}$ M. J. R. Olsson, ${ }^{171}$ A. Olszewski, ${ }^{85}$ J. Olszowska, ${ }^{85}$ Ö. O. Öncel,${ }^{24}$ D. C. O’Neill, ${ }^{152}$ A. P. O’neill, ${ }^{134}$ A. Onofre, ${ }^{139 a, 139 e}$ P. U. E. Onyisi, ${ }^{11}$ H. Oppen, ${ }^{133}$ R. G. Oreamuno Madriz, ${ }^{121}$ M. J. Oreglia, ${ }^{37}$ G. E. Orellana, ${ }^{89}$ D. Orestano,${ }^{75 a, 75 b}$ N. Orlando, ${ }^{14}$ R. S. Orr, ${ }^{167}$ V. O'Shea, ${ }^{57}$ R. Ospanov, ${ }^{60 a}$ G. Otero y Garzon, ${ }^{30}$ H. Otono, ${ }^{88}$ P. S. Ott, ${ }^{61 a}$ G. J. Ottino, ${ }^{18}$ M. Ouchrif,${ }^{35 d}$ J. Ouellette, ${ }^{29}$ F. Ould-Saada, ${ }^{133}$ A. Ouraou, ${ }^{144, a}$ Q. Ouyang, ${ }^{15 a}$ M. Owen, ${ }^{57}$ R. E. Owen, ${ }^{143}$ V. E. Ozcan, ${ }^{12 c}$ N. Ozturk, ${ }^{8}$ J. Pacalt, ${ }^{130}$ H. A. Pacey,${ }^{32}$ K. Pachal,${ }^{49}$ A. Pacheco Pages,${ }^{14}$ C. Padilla Aranda, ${ }^{14}$ S. Pagan Griso, ${ }^{18}$ G. Palacino, ${ }^{66}$ S. Palazzo ${ }^{50}$ S. Palestini,${ }^{36}$ M. Palka, ${ }^{84 b}$ P. Palni, ${ }^{84 a}$ C. E. Pandini, ${ }^{54}$ J. G. Panduro Vazquez, ${ }^{94}$ P. Pani, ${ }^{46}$ G. Panizzo, ${ }^{67 a, 67 c}$ L. Paolozzi,${ }^{54}$ C. Papadatos, ${ }^{110}$ K. Papageorgiou, ${ }^{9, p}$ S. Parajuli, ${ }^{42}$ A. Paramonov, ${ }^{6}$ C. Paraskevopoulos, ${ }^{10}$ D. Paredes Hernandez, ${ }^{63 b}$ S. R. Paredes Saenz, ${ }^{134}$ B. Parida,${ }^{180}$ T. H. Park, ${ }^{167}$ A. J. Parker, ${ }^{31}$ M. A. Parker, ${ }^{32}$ F. Parodi, ${ }^{55 b, 55 a}$ E. W. Parrish, ${ }^{121}$ J. A. Parsons, ${ }^{39}$ U. Parzefall, ${ }^{52}$ L. Pascual Dominguez, ${ }^{135}$ V. R. Pascuzzi, ${ }^{18}$ J. M. P. Pasner, ${ }^{145}$ F. Pasquali, ${ }^{120}$ E. Pasqualucci, ${ }^{73 a}$ S. Passaggio, ${ }^{55 b}$ F. Pastore,${ }^{94}$ P. Pasuwan, ${ }^{45 a, 45 b}$ S. Pataraia ${ }^{100}$ J. R. Pater, ${ }^{101}$ A. Pathak, ${ }^{181, \mathrm{e}}$ J. Patton, ${ }^{91}$ T. Pauly, ${ }^{36}$ J. Pearkes, ${ }^{153}$ M. Pedersen, ${ }^{133}$ L. Pedraza Diaz, ${ }^{119}$ R. Pedro, ${ }^{139 a}$ T. Peiffer, ${ }^{53}$ S. V. Peleganchuk, ${ }^{122 b, 122 a}$ O. Penc, ${ }^{140}$ C. Peng, ${ }^{63 b}$ H. Peng, ${ }^{60 a}$ B. S. Peralva, ${ }^{81 a}$ M. M. Perego, ${ }^{65}$ A. P. Pereira Peixoto, ${ }^{139 a}$ L. Pereira Sanchez, ${ }^{45 a, 45 b}$ D. V. Perepelitsa, ${ }^{29}$ E. Perez Codina, ${ }^{168 a}$ L. Perini, ${ }^{69 a, 69 b}$ H. Pernegger, ${ }^{36}$ S. Perrella, ${ }^{36}$ A. Perrevoort, ${ }^{120}$ K. Peters,${ }^{46}$ R. F. Y. Peters, ${ }^{101}$ B. A. Petersen, ${ }^{36}$ T. C. Petersen, ${ }^{40}$ E. Petit,${ }^{102}$ V. Petousis, ${ }^{141}$ C. Petridou, ${ }^{162}$ F. Petrucci, ${ }^{75 a, 75 b}$ M. Pettee ${ }^{183}$ N. E. Pettersson, ${ }^{103}$ K. Petukhova, ${ }^{142}$ A. Peyaud, ${ }^{144}$ R. Pezoa, ${ }^{146 d}$ L. Pezzotti, ${ }^{71 a, 71 b}$ T. Pham, ${ }^{105}$ P. W. Phillips, ${ }^{143}$ M. W. Phipps, ${ }^{173}$ G. Piacquadio, ${ }^{155}$ E. Pianori, ${ }^{18}$ A. Picazio, ${ }^{103}$ R. H. Pickles, ${ }^{101}$ R. Piegaia, ${ }^{30}$ D. Pietreanu, ${ }^{27 b}$ J. E. Pilcher, ${ }^{37}$ A. D. Pilkington, ${ }^{101}$ M. Pinamonti, ${ }^{67 a, 67 c}$ J. L. Pinfold, ${ }^{3}$ C. Pitman Donaldson, ${ }^{95}$ M. Pitt, ${ }^{161}$ L. Pizzimento ${ }^{74 a, 74 b}$ A. Pizzini, ${ }^{120}$ M.-A. Pleier, ${ }^{29}$ V. Plesanovs, ${ }^{52}$ V. Pleskot, ${ }^{142}$ E. Plotnikova,${ }^{80}$ P. Podberezko, ${ }^{122 b, 122 a}$ R. Poettgen, ${ }^{97}$ R. Poggi, ${ }^{54}$ L. Poggioli,,${ }^{135}$ I. Pogrebnyak, ${ }^{107}$ D. Pohl, ${ }^{24}$ I. Pokharel,${ }^{53}$ G. Polesello, ${ }^{71 a}$ A. Poley, ${ }^{152,168 a}$ A. Policicchio, ${ }^{73 a}, 73 b$ R. Polifka, ${ }^{142}$ A. Polini, ${ }^{23 b}$ C. S. Pollard, ${ }^{46}$ V. Polychronakos, ${ }^{29}$ D. Ponomarenko, ${ }^{112}$ L. Pontecorvo,${ }^{36}$ S. Popa,${ }^{27 a}$ G. A. Popeneciu, ${ }^{27 d}$ L. Portales, ${ }^{5}$ D. M. Portillo Quintero, ${ }^{58}$ S. Pospisil,${ }^{141}$ K. Potamianos, ${ }^{46}$ I. N. Potrap, ${ }^{80}$ C. J. Potter, ${ }^{32}$ H. Potti, ${ }^{11}$ T. Poulsen, ${ }^{97}$ J. Poveda, ${ }^{174}$ T. D. Powell, ${ }^{149}$ G. Pownall, ${ }^{46}$ M. E. Pozo Astigarraga, ${ }^{36}$ A. Prades Ibanez, ${ }^{174}$ P. Pralavorio, ${ }^{102}$ M. M. Prapa,${ }^{44}$ S. Prell, ${ }^{79}$ D. Price,${ }^{101}$ M. Primavera, ${ }^{68 a}$ M. L. Proffitt, ${ }^{148}$ N. Proklova, ${ }^{112}$ K. Prokofiev, ${ }^{63 \mathrm{c}}$ F. Prokoshin, ${ }^{80}$ S. Protopopescu, ${ }^{29}$ J. Proudfoot, ${ }^{6}$ M. Przybycien, ${ }^{84 a}$ D. Pudzha, ${ }^{137}$ A. Puri, ${ }^{173}$ P. Puzo,${ }^{65}$ D. Pyatiizbyantseva, ${ }^{112}$ J. Qian, ${ }^{106}$ Y. Qin, ${ }^{101}$ A. Quadt, ${ }^{53}$ M. Queitsch-Maitland, ${ }^{36}$ G. Rabanal Bolanos, ${ }^{59}$ M. Racko, ${ }^{28 a}$ F. Ragusa, ${ }^{69 a, 69 b}$ G. Rahal, ${ }^{98}$ J. A. Raine, ${ }^{54}$ S. Rajagopalan, ${ }^{29}$ A. Ramirez Morales,${ }^{93}$ K. Ran, ${ }^{15 a, 15 d}$ D. F. Rassloff, ${ }^{61 a}$ D. M. Rauch,${ }^{46}$ F. Rauscher, ${ }^{114}$ S. Rave, ${ }^{100}$ B. Ravina ${ }^{57}$ I. Ravinovich, ${ }^{180}$ J. H. Rawling, ${ }^{101}$ M. Raymond, ${ }^{36}$ A. L. Read, ${ }^{133}$ N. P. Readioff, ${ }^{149}$ M. Reale, ${ }^{68 a, 68 b}$ D. M. Rebuzzi ${ }^{71 a, 71 b}$ G. Redlinger, ${ }^{29}$ K. Reeves,${ }^{43}$ D. Reikher, ${ }^{161}$ A. Reiss, ${ }^{100}$ A. Rej, ${ }^{151}$ C. Rembser, ${ }^{36}$ A. Renardi, ${ }^{46}$ M. Renda ${ }^{27 b}$ M. B. Rendel, ${ }^{115}$ A. G. Rennie, ${ }^{57}$ S. Resconi ${ }^{69 a}$ E. D. Resseguie, ${ }^{18}$ S. Rettie, ${ }^{95}$ B. Reynolds, ${ }^{127}$ E. Reynolds, ${ }^{21}$ O. L. Rezanova, ${ }^{122 b, 122 a}$ P. Reznicek, ${ }^{142}$ E. Ricci, ${ }^{76 a, 76 b}$ R. Richter, ${ }^{15}$ S. Richter ${ }^{46}$ E. Richter-Was,${ }^{84 b}$ M. Ridel,,${ }^{135}$ P. Rieck, ${ }^{115}$ O. Rifki, ${ }^{46}$ M. Rijssenbeek, ${ }^{155}$ A. Rimoldi, ${ }^{71 a, 71 b}$ M. Rimoldi, ${ }^{46}$ L. Rinaldi, ${ }^{23 b}$ T. T. Rinn, ${ }^{173}$ G. Ripellino, ${ }^{154}$ I. Riu, ${ }^{14}$ P. Rivadeneira, ${ }^{46}$

J. C. Rivera Vergara, ${ }^{176}$ F. Rizatdinova, ${ }^{129}$ E. Rizvi, ${ }^{93}$ C. Rizzi, ${ }^{36}$ S. H. Robertson, ${ }^{104,1}$ M. Robin, ${ }^{46}$ D. Robinson, ${ }^{32}$ C. M. Robles Gajardo, ${ }^{146 \mathrm{~d}}$ M. Robles Manzano, ${ }^{100}$ A. Robson, ${ }^{57}$ A. Rocchi ${ }^{74 a, 74 b}$ C. Roda, ${ }^{72 a, 72 b}$ S. Rodriguez Bosca, ${ }^{174}$ A. Rodriguez Rodriguez, ${ }^{52}$ A. M. Rodríguez Vera, ${ }^{168 b}$ S. Roe, ${ }^{36}$ J. Roggel, ${ }^{182}$ O. Røhne, ${ }^{133}$ R. Röhrig, ${ }^{115}$ R. A. Rojas, ${ }^{146 \mathrm{~d}}$ B. Roland, ${ }^{52}$ C. P. A. Roland, ${ }^{66}$ J. Roloff, ${ }^{29}$ A. Romaniouk, ${ }^{112}$ M. Romano, ${ }^{23 b, 23 a}$ N. Rompotis, ${ }^{91}$ M. Ronzani, ${ }^{125}$ L. Roos, ${ }^{135}$ S. Rosati, ${ }^{73 a}$ G. Rosin, ${ }^{103}$ B. J. Rosser, ${ }^{136}$ E. Rossi, ${ }^{46}$ E. Rossi, ${ }^{75 a, 75 b}$ E. Rossi, ${ }^{70 a, 70 b}$ L. P. Rossi, ${ }^{55 b}$ L. Rossini, ${ }^{46}$ R. Rosten, ${ }^{14}$ M. Rotaru, ${ }^{27 b}$ B. Rottler, ${ }^{52}$ D. Rousseau, ${ }^{65}$ G. Rovelli, ${ }^{71 a, 71 b}$ A. Roy, ${ }^{11}$ D. Roy,${ }^{33 \mathrm{e}}$ A. Rozanov, ${ }^{102}$ Y. Rozen, ${ }^{160}$ X. Ruan, ${ }^{33 \mathrm{e}}$

T. A. Ruggeri, ${ }^{1}$ F. Rühr, ${ }^{52}$ A. Ruiz-Martinez, ${ }^{174}$ A. Rummler ${ }^{36}$ Z. Rurikova, ${ }^{52}$ N. A. Rusakovich ${ }^{80}$ H. L. Russell, ${ }^{104}$ L. Rustige, ${ }^{38,47}$ J. P. Rutherfoord, ${ }^{7}$ E. M. Rüttinger, ${ }^{149}$ M. Rybar, ${ }^{142}$ G. Rybkin, ${ }^{65}$ E. B. Rye, ${ }^{133}$ A. Ryzhov, ${ }^{123}$ 
J. A. Sabater Iglesias, ${ }^{46}$ P. Sabatini, ${ }^{174}$ L. Sabetta, ${ }^{73 a, 73 b}$ S. Sacerdoti, ${ }^{65}$ H. F-W. Sadrozinski, ${ }^{145}$ R. Sadykov, ${ }^{80}$

F. Safai Tehrani, ${ }^{73 a}$ B. Safarzadeh Samani, ${ }^{156}$ M. Safdari, ${ }^{153}$ P. Saha, ${ }^{121}$ S. Saha,${ }^{104}$ M. Sahinsoy, ${ }^{115}$ A. Sahu, ${ }^{182}$ M. Saimpert, ${ }^{36}$ M. Saito, ${ }^{163}$ T. Saito, ${ }^{163}$ H. Sakamoto, ${ }^{163}$ D. Salamani,${ }^{54}$ G. Salamanna, ${ }^{75 a, 75 b}$ A. Salnikov, ${ }^{153}$ J. Salt, ${ }^{174}$ A. Salvador Salas, ${ }^{14}$ D. Salvatore, ${ }^{41 b, 41 a}$ F. Salvatore, ${ }^{156}$ A. Salvucci, ${ }^{63 a}$ A. Salzburger, ${ }^{36}$ J. Samarati, ${ }^{36}$ D. Sammel, ${ }^{52}$ D. Sampsonidis, ${ }^{162}$ D. Sampsonidou, ${ }^{60,60 c}$ J. Sánchez, ${ }^{174}$ A. Sanchez Pineda,${ }^{67,36,67 c}$ H. Sandaker ${ }^{133}$ C. O. Sander ${ }^{46}$ I. G. Sanderswood, ${ }^{90}$ M. Sandhoff, ${ }^{182}$ C. Sandoval, ${ }^{22 b}$ D. P. C. Sankey, ${ }^{143}$ M. Sannino, ${ }^{55,55 a}$ Y. Sano, ${ }^{117}$ A. Sansoni, ${ }^{51}$ C. Santoni, ${ }^{38}$ H. Santos, ${ }^{139 a, 139 b}$ S. N. Santpur, ${ }^{18}$ A. Santra, ${ }^{174}$ K. A. Saoucha, ${ }^{149}$ A. Sapronov, ${ }^{80}$ J. G. Saraiva, ${ }^{139 a, 139 d}$ O. Sasaki, ${ }^{82}$ K. Sato, ${ }^{169}$ F. Sauerburger, ${ }^{52}$ E. Sauvan, ${ }^{5}$ P. Savard,${ }^{167, d}$ R. Sawada, ${ }^{163}$ C. Sawyer, ${ }^{143}$ L. Sawyer, ${ }^{96}$ I. Sayago Galvan, ${ }^{174}$ C. Sbarra, ${ }^{23 b}$ A. Sbrizzi, ${ }^{67 a, 67 \mathrm{c}}$ T. Scanlon, ${ }^{95}$ J. Schaarschmidt, ${ }^{148}$ P. Schacht, ${ }^{115}$ D. Schaefer, ${ }^{37}$ L. Schaefer, ${ }^{136}$ U. Schäfer, ${ }^{100}$ A. C. Schaffer, ${ }^{65}$ D. Schaile, ${ }^{114}$ R. D. Schamberger, ${ }^{155}$ E. Schanet, ${ }^{114}$ C. Scharf, ${ }^{19}$ N. Scharmberg, ${ }^{101}$ V. A. Schegelsky, ${ }^{137}$ D. Scheirich, ${ }^{142}$ F. Schenck, ${ }^{19}$ M. Schernau, ${ }^{171}$ C. Schiavi, ${ }^{55 b, 55 a}$ L. K. Schildgen, ${ }^{24}$ Z. M. Schillaci, ${ }^{26}$ E. J. Schioppa ${ }^{68 a, 68 b}$ M. Schioppa, ${ }^{41 b, 41 a}$ K. E. Schleicher, ${ }^{52}$ S. Schlenker,${ }^{36}$ K. R. Schmidt-Sommerfeld, ${ }^{115}$ K. Schmieden, ${ }^{100}$ C. Schmitt, ${ }^{100}$ S. Schmitt, ${ }^{46}$ L. Schoeffel, ${ }^{144}$ A. Schoening, ${ }^{61 b}$ P. G. Scholer,${ }^{52}$ E. Schopf,${ }^{134}$ M. Schott, ${ }^{100}$ J. F. P. Schouwenberg, ${ }^{119}$ J. Schovancova, ${ }^{36}$ S. Schramm, ${ }^{54}$ F. Schroeder, ${ }^{182}$ A. Schulte, ${ }^{100}$ H-C. Schultz-Coulon, ${ }^{61 a}$ M. Schumacher, ${ }^{52}$ B. A. Schumm, ${ }^{145}$ Ph. Schune, ${ }^{144}$ A. Schwartzman, ${ }^{153}$ T. A. Schwarz, ${ }^{106} \mathrm{Ph}$. Schwemling, ${ }^{144}$ R. Schwienhorst, ${ }^{107}$ A. Sciandra, ${ }^{145}$ G. Sciolla, ${ }^{26}$ F. Scuri, ${ }^{72 a}$ F. Scutti, ${ }^{105}$ L. M. Scyboz, ${ }^{115}$ C. D. Sebastiani, ${ }^{91}$ K. Sedlaczek, ${ }^{47}$ P. Seema, ${ }^{19}$ S. C. Seidel, ${ }^{118}$ A. Seiden, ${ }^{145}$ B. D. Seidlitz ${ }^{29}$ T. Seiss, ${ }^{37}$ C. Seitz, ${ }^{46}$ J. M. Seixas, ${ }^{81 b}$ G. Sekhniaidze ${ }^{70 a}$ S. J. Sekula, ${ }^{42}$ N. Semprini-Cesari, ${ }^{23 b, 23 a}$ S. Sen, ${ }^{49}$ C. Serfon, ${ }^{29}$ L. Serin,${ }^{65}$ L. Serkin, ${ }^{67 a, 67 b}$ M. Sessa, ${ }^{60 a}$ H. Severini, ${ }^{128}$ S. Sevova, ${ }^{153}$ F. Sforza, ${ }^{55 b, 55 a}$ A. Sfyrla, ${ }^{54}$ E. Shabalina, ${ }^{53}$ J. D. Shahinian, ${ }^{136}$ N. W. Shaikh, ${ }^{45 a, 45 b}$ D. Shaked Renous, ${ }^{180}$ L. Y. Shan, ${ }^{15 a}$ M. Shapiro, ${ }^{18}$ A. Sharma, ${ }^{36}$ A. S. Sharma, ${ }^{1}$ P. B. Shatalov, ${ }^{124}$ K. Shaw, ${ }^{156}$ S. M. Shaw, ${ }^{101}$ M. Shehade, ${ }^{180}$ Y. Shen, ${ }^{128}$ A. D. Sherman, ${ }^{25}$ P. Sherwood,,${ }^{95}$ L. Shi, ${ }^{95}$ C. O. Shimmin, ${ }^{183}$ Y. Shimogama, ${ }^{179}$ M. Shimojima, ${ }^{116}$ J. D. Shinner, ${ }^{94}$ I. P. J. Shipsey, ${ }^{134}$ S. Shirabe, ${ }^{165}$ M. Shiyakova, ${ }^{80, f f}$ J. Shlomi, ${ }^{180}$ A. Shmeleva, ${ }^{111}$ M. J. Shochet, ${ }^{37}$ J. Shojaii, ${ }^{105}$ D. R. Shope, ${ }^{154}$ S. Shrestha, ${ }^{127}$ E. M. Shrif, ${ }^{33 e}$ M. J. Shroff, ${ }^{176}$ E. Shulga, ${ }^{180}$ P. Sicho, ${ }^{140}$ A. M. Sickles, ${ }^{173}$ E. Sideras Haddad, ${ }^{33 e}$ O. Sidiropoulou, ${ }^{36}$ A. Sidoti, ${ }^{23 b, 23 a}$ F. Siegert, ${ }^{48}$ Dj. Sijacki, ${ }^{16}$ M. Silva Jr., ${ }^{181}$ M. V. Silva Oliveira, ${ }^{36}$ S. B. Silverstein, ${ }^{45 a}$ S. Simion, ${ }^{65}$ R. Simoniello, ${ }^{100}$ C. J. Simpson-allsop, ${ }^{21}$ S. Simsek, ${ }^{12 b}$ P. Sinervo, ${ }^{167}$ V. Sinetckii, ${ }^{113}$ S. Singh, ${ }^{152}$ S. Sinha, ${ }^{33 e}$ M. Sioli, ${ }^{23 b, 23 a}$ I. Siral, ${ }^{131}$ S. Yu. Sivoklokov, ${ }^{113}$ J. Sjölin, ${ }^{45 a, 45 b}$ A. Skaf, ${ }^{53}$ E. Skorda, ${ }^{97}$ P. Skubic, ${ }^{128}$ M. Slawinska, ${ }^{85}$ K. Sliwa ${ }^{170}$ V. Smakhtin, ${ }^{180}$ B. H. Smart ${ }^{143}$ J. Smiesko, ${ }^{28 b}$ N. Smirnov, ${ }^{112}$ S. Yu. Smirnov, ${ }^{112}$ Y. Smirnov, ${ }^{112}$ L. N. Smirnova, ${ }^{113, g g}$ O. Smirnova, ${ }^{97}$ E. A. Smith ${ }^{37}$ H. A. Smith, ${ }^{134}$ M. Smizanska, ${ }^{90}$ K. Smolek, ${ }^{141}$ A. Smykiewicz, ${ }^{85}$ A. A. Snesarev, ${ }^{111}$ H. L. Snoek, ${ }^{120}$ I. M. Snyder, ${ }^{131}$ S. Snyder, ${ }^{29}$ R. Sobie, ${ }^{176,1}$ A. Soffer, ${ }^{161}$ A. Søgaard ${ }^{50}$ F. Sohns, ${ }^{53}$ C. A. Solans Sanchez, ${ }^{36}$ E. Yu. Soldatov, ${ }^{112}$ U. Soldevila, ${ }^{174}$ A. A. Solodkov, ${ }^{123}$ A. Soloshenko, ${ }^{80}$ O. V. Solovyanov, ${ }^{123}$ V. Solovyev, ${ }^{137}$ P. Sommer, ${ }^{149}$ H. Son, ${ }^{170}$ A. Sonay ${ }^{14}$ W. Song, ${ }^{143}$ W. Y. Song, ${ }^{168 b}$ A. Sopczak, ${ }^{141}$ A. L. Sopio, ${ }^{95}$ F. Sopkova, ${ }^{28 b}$ S. Sottocornola ${ }^{71 a, 71 b}$ R. Soualah, ${ }^{67 a, 67 c}$ A. M. Soukharev, ${ }^{122 b, 122 a}$ D. South, ${ }^{46}$

S. Spagnolo, ${ }^{68 a, 68 b}$ M. Spalla, ${ }^{115}$ M. Spangenberg, ${ }^{178}$ F. Spanò,${ }^{94}$ D. Sperlich,${ }^{52}$ T. M. Spieker,${ }^{61 a}$ G. Spigo, ${ }^{36}$ M. Spina, ${ }^{156}$ D. P. Spiteri ${ }^{57}$ M. Spousta, ${ }^{142}$ A. Stabile, ${ }^{69 a, 69 b}$ B. L. Stamas, ${ }^{121}$ R. Stamen, ${ }^{61 a}$ M. Stamenkovic, ${ }^{120}$ A. Stampekis ${ }^{21}$ E. Stanecka, ${ }^{85}$ B. Stanislaus, ${ }^{134}$ M. M. Stanitzki, ${ }^{46}$ M. Stankaityte, ${ }^{134}$ B. Stapf, ${ }^{120}$ E. A. Starchenko, ${ }^{123}$ G. H. Stark, ${ }^{145}$ J. Stark, ${ }^{58}$ P. Staroba, ${ }^{140}$ P. Starovoitov, ${ }^{61 a}$ S. Stärz, ${ }^{104}$ R. Staszewski, ${ }^{85}$ G. Stavropoulos, ${ }^{44}$ M. Stegler, ${ }^{46}$ P. Steinberg, ${ }^{29}$

A. L. Steinhebel, ${ }^{131}$ B. Stelzer, ${ }^{152,168 a}$ H. J. Stelzer, ${ }^{138}$ O. Stelzer-Chilton, ${ }^{168 a}$ H. Stenzel, ${ }^{56}$ T. J. Stevenson, ${ }^{156}$

G. A. Stewart, ${ }^{36}$ M. C. Stockton, ${ }^{36}$ G. Stoicea,${ }^{27 b}$ M. Stolarski, ${ }^{139 a}$ S. Stonjek, ${ }^{115}$ A. Straessner, ${ }^{48}$ J. Strandberg, ${ }^{154}$ S. Strandberg, ${ }^{45 a, 45 b}$ M. Strauss, ${ }^{128}$ T. Strebler, ${ }^{102}$ P. Strizenec ${ }^{28 b}$ R. Ströhmer, ${ }^{177}$ D. M. Strom, ${ }^{131}$ R. Stroynowski, ${ }^{42}$ A. Strubig, ${ }^{45 a, 45 b}$ S. A. Stucci, ${ }^{29}$ B. Stugu, ${ }^{17}$ J. Stupak, ${ }^{128}$ N. A. Styles,${ }^{46}$ D. Su, ${ }^{153}$ W. Su, ${ }^{60,, 148,60 c}$ X. Su, ${ }^{60 a}$ N. B. Suarez, ${ }^{138}$ V. V. Sulin, ${ }^{111}$ M. J. Sullivan, ${ }^{91}$ D. M. S. Sultan, ${ }^{54}$ S. Sultansoy, ${ }^{4 c}$ T. Sumida, ${ }^{86}$ S. Sun, ${ }^{106}$ X. Sun, ${ }^{101}$ C. J. E. Suster, ${ }^{157}$ M. R. Sutton, ${ }^{156}$ S. Suzuki, ${ }^{82}$ M. Svatos, ${ }^{140}$ M. Swiatlowski, ${ }^{168 a}$ S. P. Swift, ${ }^{2}$ T. Swirski, ${ }^{177}$ A. Sydorenko, ${ }^{100}$ I. Sykora, ${ }^{28 a}$ M. Sykora, ${ }^{142}$ T. Sykora, ${ }^{142}$ D. Ta, ${ }^{100}$ K. Tackmann, ${ }^{46, h}$ J. Taenzer, ${ }^{161}$ A. Taffard,${ }^{171}$ R. Tafirout, ${ }^{168 a}$ E. Tagiev, ${ }^{123}$

R. H. M. Taibah, ${ }^{135}$ R. Takashima, ${ }^{87}$ K. Takeda,${ }^{83}$ T. Takeshita, ${ }^{150}$ E. P. Takeva,${ }^{50}$ Y. Takubo,${ }^{82}$ M. Talby, ${ }^{102}$ A. A. Talyshev, ${ }^{122 b, 122 a}$ K. C. Tam,${ }^{6 b}$ N. M. Tamir,${ }^{161}$ J. Tanaka, ${ }^{163}$ R. Tanaka ${ }^{65}$ S. Tapia Araya, ${ }^{173}$ S. Tapprogge, ${ }^{100}$ A. Tarek Abouelfadl Mohamed, ${ }^{107}$ S. Tarem, ${ }^{160}$ K. Tariq ${ }^{60 b}$ G. Tarna, ${ }^{27 b, i i}$ G. F. Tartarelli, ${ }^{69 a}$ P. Tas, ${ }^{142}$ M. Tasevsky, ${ }^{140}$ E. Tassi,${ }^{41 b, 41 \mathrm{a}}$ G. Tateno, ${ }^{163}$ A. Tavares Delgado,${ }^{139 \mathrm{a}}$ Y. Tayalati, ${ }^{35 \mathrm{e}}$ A. J. Taylor, ${ }^{50}$ G. N. Taylor, ${ }^{105}$ W. Taylor, ${ }^{168 \mathrm{~b}}$ H. Teagle, ${ }^{91}$ A. S. Tee, ${ }^{90}$ R. Teixeira De Lima, ${ }^{153}$ P. Teixeira-Dias, ${ }^{94}$ H. Ten Kate, ${ }^{36}$ J. J. Teoh, ${ }^{120}$ K. Terashi, ${ }^{163}$ J. Terron, ${ }^{99}$ S. Terzo, ${ }^{14}$ 
M. Testa, ${ }^{51}$ R. J. Teuscher, ${ }^{167,1}$ N. Themistokleous, ${ }^{50}$ T. Theveneaux-Pelzer, ${ }^{19}$ D. W. Thomas, ${ }^{94}$ J.P. Thomas, ${ }^{21}$ E. A. Thompson, ${ }^{46}$ P. D. Thompson, ${ }^{21}$ E. Thomson, ${ }^{136}$ E. J. Thorpe,${ }^{93}$ V. O. Tikhomirov, ${ }^{11, j j}$ Yu. A. Tikhonov, ${ }^{122 b, 122 a}$ S. Timoshenko, ${ }^{112}$ P. Tipton, ${ }^{183}$ S. Tisserant ${ }^{102}$ K. Todome, ${ }^{23 b, 23 a}$ S. Todorova-Nova, ${ }^{142}$ S. Todt,${ }^{48}$ J. Tojo, ${ }^{88}$ S. Tokár, ${ }^{28 a}$ K. Tokushuku, ${ }^{82}$ E. Tolley, ${ }^{127}$ R. Tombs, ${ }^{32}$ K. G. Tomiwa, ${ }^{33 e}$ M. Tomoto,${ }^{82,117}$ L. Tompkins, ${ }^{153}$ P. Tornambe,${ }^{103}$ E. Torrence, ${ }^{131}$ H. Torres,${ }^{48}$ E. Torró Pastor, ${ }^{174}$ M. Toscani, ${ }^{30}$ C. Tosciri, ${ }^{134}$ J. Toth, ${ }^{102, k k}$ D. R. Tovey, ${ }^{149}$ A. Traeet ${ }^{17}$ C. J. Treado ${ }^{125}$ T. Trefzger, ${ }^{177}$ F. Tresoldi ${ }^{156}$ A. Tricoli, ${ }^{29}$ I. M. Trigger ${ }^{168 a}$ S. Trincaz-Duvoid, ${ }^{135}$ D. A. Trischuk,${ }^{175}$ W. Trischuk ${ }^{167}$ B. Trocmé, ${ }^{58}$ A. Trofymov,${ }^{65}$ C. Troncon, ${ }^{69 a}$ F. Trovato, ${ }^{156}$ L. Truong, ${ }^{33 c}$ M. Trzebinski, ${ }^{85}$ A. Trzupek, ${ }^{85}$ F. Tsai, ${ }^{46}$ P. V. Tsiareshka, ${ }^{108, y}$ A. Tsirigotis, ${ }^{162, z}$ V. Tsiskaridze, ${ }^{155}$ E. G. Tskhadadze, ${ }^{159 a}$ M. Tsopoulou, ${ }^{162}$ I. I. Tsukerman, ${ }^{124}$ V. Tsulaia, ${ }^{18}$ S. Tsuno, ${ }^{82} \mathrm{D}$. Tsybychev, ${ }^{155} \mathrm{Y}$. Tu, ${ }^{6 \mathrm{~b}}$ A. Tudorache, ${ }^{27 \mathrm{~b}} \mathrm{~V}$. Tudorache ${ }^{27 \mathrm{~b}}$ A. N. Tuna, ${ }^{36}$ S. Turchikhin ${ }^{80}$ D. Turgeman, ${ }^{180}$ I. Turk Cakir, ${ }^{4 b, 11}$ R. J. Turner, ${ }^{21}$ R. Turra, ${ }^{69 a}$ P. M. Tuts, ${ }^{39}$ S. Tzamarias, ${ }^{162}$ E. Tzovara, ${ }^{100}$ K. Uchida, ${ }^{163}$ F. Ukegawa, ${ }^{169}$ G. Unal,${ }^{36}$ M. Unal, ${ }^{11}$ A. Undrus, ${ }^{29}$ G. Unel, ${ }^{171}$ F. C. Ungaro, ${ }^{105}$ Y. Unno, ${ }^{82}$ K. Uno, ${ }^{163}$ J. Urban, ${ }^{28 b}$ P. Urquijo, ${ }^{105}$ G. Usai,${ }^{8}$ Z. Uysal, ${ }^{12 \mathrm{~d}}$ V. Vacek,${ }^{141}$ B. Vachon,${ }^{104}$ K. O. H. Vadla,${ }^{133}$ T. Vafeiadis,${ }^{36}$ A. Vaidya,${ }^{95}$ C. Valderanis, ${ }^{114}$ E. Valdes Santurio, ${ }^{45 a, 45 b}$ M. Valente, ${ }^{168 a}$ S. Valentinetti, ${ }^{23 b, 23 a}$ A. Valero, ${ }^{174}$ L. Valéry, ${ }^{46}$ R. A. Vallance, ${ }^{21}$ A. Vallier, ${ }^{36}$ J. A. Valls Ferrer, ${ }^{174}$ T. R. Van Daalen, ${ }^{14}$ P. Van Gemmeren, ${ }^{6}$ S. Van Stroud, ${ }^{95}$ I. Van Vulpen, ${ }^{120}$ M. Vanadia, ${ }^{74 a, 74 b}$ W. Vandelli, ${ }^{36}$ M. Vandenbroucke, ${ }^{144}$ E. R. Vandewall, ${ }^{129}$ D. Vannicola, ${ }^{73 a, 73 b}$ R. Vari, ${ }^{73 a}$ E. W. Varnes, ${ }^{7}$ C. Varni, ${ }^{55 b, 55 a}$ T. Varol, ${ }^{158}$ D. Varouchas, ${ }^{65}$ K. E. Varvell, ${ }^{157}$ M. E. Vasile, ${ }^{27 b}$ G. A. Vasquez, ${ }^{176}$ F. Vazeille, ${ }^{38}$

D. Vazquez Furelos, ${ }^{14}$ T. Vazquez Schroeder, ${ }^{36}$ J. Veatch, ${ }^{53}$ V. Vecchio, ${ }^{101}$ M. J. Veen, ${ }^{120}$ L. M. Veloce,${ }^{167}$ F. Veloso, ${ }^{139 a, 139 c}$ S. Veneziano, ${ }^{73 a}$ A. Ventura, ${ }^{6 a, 68 b}$ A. Verbytskyi, ${ }^{115}$ V. Vercesi, ${ }^{71 a}$ M. Verducci, ${ }^{72 a, 72 b}$ C. M. Vergel Infante,${ }^{79}$ C. Vergis,${ }^{24}$ W. Verkerke, ${ }^{120}$ A. T. Vermeulen, ${ }^{120}$ J. C. Vermeulen, ${ }^{120}$ C. Vernieri, ${ }^{153}$ P. J. Verschuuren, ${ }^{94}$ M. C. Vetterli, ${ }^{152, d}$ N. Viaux Maira, ${ }^{146 \mathrm{~d}}$ T. Vickey, ${ }^{149}$ O. E. Vickey Boeriu, ${ }^{149}$ G. H. A. Viehhauser, ${ }^{134}$ L. Vigani, ${ }^{61 b}$ M. Villa, ${ }^{23 b, 23 a}$ M. Villaplana Perez, ${ }^{174}$ E. M. Villhauer, ${ }^{50}$ E. Vilucchi,${ }^{51}$ M. G. Vincter, ${ }^{34}$ G. S. Virdee,${ }^{21}$ A. Vishwakarma, ${ }^{50}$ C. Vittori, ${ }^{23 b, 23 a}$ I. Vivarelli, ${ }^{156}$ M. Vogel, ${ }^{182}$ P. Vokac, ${ }^{141}$ J. Von Ahnen, ${ }^{46}$ S. E. von Buddenbrock, ${ }^{33 e}$ E. Von Toerne,${ }^{24}$ V. Vorobel,${ }^{142}$ K. Vorobev,${ }^{112}$ M. Vos, ${ }^{174}$ J. H. Vossebeld,${ }^{91}$ M. Vozak, ${ }^{101}$ N. Vranjes, ${ }^{16}$ M. Vranjes Milosavljevic, ${ }^{16}$ V. Vrba, ${ }^{141}$ M. Vreeswijk, ${ }^{120}$ N. K. Vu, ${ }^{102}$ R. Vuillermet, ${ }^{36}$ I. Vukotic, ${ }^{37}$ S. Wada, ${ }^{169}$ P. Wagner, ${ }^{24}$ W. Wagner, ${ }^{182}$ J. Wagner-Kuhr, ${ }^{114}$ S. Wahdan, ${ }^{182}$ H. Wahlberg, ${ }^{89}$ R. Wakasa, ${ }^{169}$ V. M. Walbrecht, ${ }^{115}$ J. Walder, ${ }^{143}$ R. Walker, ${ }^{114}$ S. D. Walker, ${ }^{94}$ W. Walkowiak, ${ }^{151}$ V. Wallangen, ${ }^{45 a, 45 b}$ A. M. Wang, ${ }^{59}$ A. Z. Wang, ${ }^{181}$ C. Wang, ${ }^{60 a}$ C. Wang, ${ }^{60 \mathrm{c}} \mathrm{H}$. Wang, ${ }^{18} \mathrm{H}$. Wang, ${ }^{3}$ J. Wang, ${ }^{63 a}$ P. Wang, ${ }^{42}$ Q. Wang, ${ }^{128}$ R.-J. Wang, ${ }^{100}$ R. Wang, ${ }^{60 a}$ R. Wang, ${ }^{6}$ S. M. Wang, ${ }^{158}$ W. T. Wang, ${ }^{60 a}$ W. Wang, ${ }^{15 c}$ W. X. Wang, ${ }^{60 a}$ Y. Wang, ${ }^{60 a}$ Z. Wang, ${ }^{106}$ C. Wanotayaroj, ${ }^{46}$ A. Warburton, ${ }^{104}$ C. P. Ward ${ }^{32}$ R. J. Ward, ${ }^{21}$ N. Warrack, ${ }^{57}$ A. T. Watson, ${ }^{21}$ M. F. Watson, ${ }^{21}$ G. Watts, ${ }^{148}$ B. M. Waugh, ${ }^{95}$ A. F. Webb,${ }^{11}$ C. Weber, ${ }^{29}$ M. S. Weber, ${ }^{20}$ S. A. Weber, ${ }^{34}$ S. M. Weber, ${ }^{61 a}$ Y. Wei, ${ }^{134}$ A. R. Weidberg, ${ }^{134}$ J. Weingarten, ${ }^{47}$ M. Weirich, ${ }^{100}$ C. Weiser, ${ }^{52}$ P. S. Wells,${ }^{36}$ T. Wenaus, ${ }^{29}$ B. Wendland, ${ }^{47}$ T. Wengler, ${ }^{36}$ S. Wenig,${ }^{36}$ N. Wermes,${ }^{24}$ M. Wessels, ${ }^{61 a}$ T. D. Weston, ${ }^{20}$ K. Whalen, ${ }^{131}$ A. M. Wharton, ${ }^{90}$ A. S. White, ${ }^{106}$ A. White, ${ }^{8}$ M. J. White, ${ }^{1}$ D. Whiteson, ${ }^{171}$ B. W. Whitmore, ${ }^{90}$ W. Wiedenmann, ${ }^{181}$ C. Wiel, ${ }^{48}$ M. Wielers, ${ }^{143}$

N. Wieseotte, ${ }^{100}$ C. Wiglesworth, ${ }^{40}$ L. A. M. Wiik-Fuchs, ${ }^{52}$ H. G. Wilkens, ${ }^{36}$ L. J. Wilkins, ${ }^{94}$ D. M. Williams, ${ }^{39}$ H. H. Williams, ${ }^{136}$ S. Williams,${ }^{32}$ S. Willocq, ${ }^{103}$ P. J. Windischhofer, ${ }^{134}$ I. Wingerter-Seez, ${ }^{5}$ E. Winkels, ${ }^{156}$ F. Winklmeier, ${ }^{131}$ B. T. Winter, ${ }^{52}$ M. Wittgen, ${ }^{153}$ M. Wobisch, ${ }^{96}$ A. Wolf, ${ }^{100}$ R. Wölker, ${ }^{134}$ J. Wollrath, ${ }^{52}$ M. W. Wolter, ${ }^{85}$ H. Wolters, ${ }^{139 a, 139 c}$ V. W. S. Wong, ${ }^{175}$ A. F. Wongel, ${ }^{46}$ N. L. Woods, ${ }^{145}$ S. D. Worm,${ }^{46}$ B. K. Wosiek,${ }^{85}$ K. W. Woźniak, ${ }^{85}$ K. Wraight, ${ }^{57}$ S. L. Wu, ${ }^{181}$ X. Wu,${ }^{54}$ Y. Wu, ${ }^{60 a}$ J. Wuerzinger ${ }^{134}$ T. R. Wyatt, ${ }^{101}$ B. M. Wynne, ${ }^{50}$ S. Xella, ${ }^{40}$ L. Xia, ${ }^{178}$ J. Xiang, ${ }^{63 c}$ X. Xiao, ${ }^{106}$ X. Xie, ${ }^{60 a}$ I. Xiotidis, ${ }^{156}$ D. Xu, ${ }^{15 a}$ H. Xu ${ }^{60 a}$ H. Xu, ${ }^{60 a}$ L. Xu, ${ }^{29}$ R. Xu, ${ }^{136}$ T. Xu, ${ }^{144}$ W. Xu, ${ }^{106}$ Y. Xu, ${ }^{15 b}$ Z. Xu, ${ }^{60 b}$ Z. Xu, ${ }^{153}$ B. Yabsley,${ }^{157}$ S. Yacoob, ${ }^{33 a}$ D. P. Yallup,${ }^{95}$ N. Yamaguchi, ${ }^{88}$ Y. Yamaguchi,${ }^{165}$ A. Yamamoto, ${ }^{82}$ M. Yamatani, ${ }^{163}$ T. Yamazaki, ${ }^{163}$ Y. Yamazaki, ${ }^{83}$ J. Yan, ${ }^{60 c}$ Z. Yan, ${ }^{25}$ H. J. Yang, ${ }^{60 c, 60 d}$ H. T. Yang, ${ }^{18}$ S. Yang, ${ }^{60 a}$ T. Yang, ${ }^{63 c}$ X. Yang,${ }^{60 a}$ X. Yang, ${ }^{60 b, 58}$ Y. Yang, ${ }^{163}$ Z. Yang, ${ }^{60 a}$ W-M. Yao, ${ }^{18}$ Y. C. Yap,${ }^{46}$ H. Ye, ${ }^{15 c}$ J. Ye ${ }^{42}$ S. Ye, ${ }^{29}$ I. Yeletskikh, ${ }^{80}$ M. R. Yexley, ${ }^{90}$ E. Yigitbasi, ${ }^{25}$ P. Yin,${ }^{39}$ K. Yorita, ${ }^{179}$ K. Yoshihara, ${ }^{79}$ C. J. S. Young, ${ }^{36}$ C. Young, ${ }^{153}$ J. Yu, ${ }^{79}$ R. Yuan, ${ }^{60 b, m m}$ X. Yue,${ }^{61 a}$ M. Zaazoua ${ }^{35 \mathrm{e}}$ B. Zabinski, ${ }^{85}$ G. Zacharis, ${ }^{10}$ E. Zaffaroni, ${ }^{54}$ J. Zahreddine, ${ }^{135}$ A. M. Zaitsev, ${ }^{123, \mathrm{i}}$ T. Zakareishvili, ${ }^{159 \mathrm{~b}}$ N. Zakharchuk, ${ }^{34}$ S. Zambito, ${ }^{36}$ D. Zanzi, ${ }^{36}$ S. V. Zeißner, ${ }^{47}$ C. Zeitnitz, ${ }^{182}$ G. Zemaityte, ${ }^{134}$ J. C. Zeng, ${ }^{173}$ O. Zenin, ${ }^{123}$ T. Ženiš, ${ }^{28 \mathrm{a}}$ D. Zerwas, ${ }^{65}$ M. Zgubič,${ }^{134}$ B. Zhang, ${ }^{15 \mathrm{c}}$ D. F. Zhang, ${ }^{15 \mathrm{~b}}$ G. Zhang, ${ }^{15 \mathrm{~b}}$ J. Zhang, ${ }^{6}$ Kaili. Zhang, ${ }^{15 \mathrm{a}}$ L. Zhang, ${ }^{15 \mathrm{c}}$ L. Zhang, ${ }^{60 \mathrm{a}}$ M. Zhang, ${ }^{173}$ R. Zhang, ${ }^{181}$ S. Zhang, ${ }^{106}$ X. Zhang, ${ }^{60 \mathrm{c}}$ X. Zhang, ${ }^{60 \mathrm{~b}}$ Y. Zhang, ${ }^{15 \mathrm{a}, 15 \mathrm{~d}} \mathrm{Z}$. Zhang, ${ }^{63 \mathrm{a}} \mathrm{Z}$. Zhang, ${ }^{65}$ P. Zhao, ${ }^{49}$ Y. Zhao, ${ }^{145}$ Z. Zhao, ${ }^{60 a}$ A. Zhemchugov ${ }^{80}$ Z. Zheng, ${ }^{106}$ D. Zhong, ${ }^{173}$ B. Zhou, ${ }^{106}$ C. Zhou, ${ }^{181}$ H. Zhou, ${ }^{7}$ M. Zhou, ${ }^{155}$ N. Zhou, ${ }^{60 \mathrm{c}}$ Y. Zhou, ${ }^{7}$ C. G. Zhu, ${ }^{60 \mathrm{~b}}$ C. Zhu, ${ }^{15 \mathrm{a}, 15 \mathrm{~d}}$ H. L. Zhu, ${ }^{60 \mathrm{a}}$ H. Zhu, ${ }^{15 \mathrm{a}}$ J. Zhu, ${ }^{106}$ Y. Zhu ${ }^{60 \mathrm{a}}$ X. Zhuang, ${ }^{15 \mathrm{a}}$ 
K. Zhukov, ${ }^{111}$ V. Zhulanov, ${ }^{122 b, 122 a}$ D. Zieminska, ${ }^{66}$ N. I. Zimine, ${ }^{80}$ S. Zimmermann, ${ }^{52}$ Z. Zinonos, ${ }^{115}$ M. Ziolkowski, ${ }^{151}$ L. Živković, ${ }^{16}$ G. Zobernig, ${ }^{181}$ A. Zoccoli, ${ }^{23 b, 23 a}$ K. Zoch, ${ }^{53}$ T. G. Zorbas, ${ }^{149}$ R. Zou, ${ }^{37}$ and L. Zwalinski ${ }^{36}$

(ATLAS Collaboration)

${ }^{1}$ Department of Physics, University of Adelaide, Adelaide, Australia

${ }^{2}$ Physics Department, SUNY Albany, Albany, New York, USA

${ }^{3}$ Department of Physics, University of Alberta, Edmonton, Alberta, Canada

${ }^{4 a}$ Department of Physics, Ankara University, Ankara, Turkey

${ }^{4 \mathrm{~b}}$ Istanbul Aydin University, Application and Research Center for Advanced Studies, Istanbul, Turkey

${ }^{4 \mathrm{c}}$ Division of Physics, TOBB University of Economics and Technology, Ankara, Turkey

${ }^{5}$ LAPP, Université Grenoble Alpes, Université Savoie Mont Blanc, CNRS/IN2P3, Annecy, France

${ }^{6}$ High Energy Physics Division, Argonne National Laboratory, Argonne, Illinois, USA

${ }^{7}$ Department of Physics, University of Arizona, Tucson, Arizona, USA

${ }^{8}$ Department of Physics, University of Texas at Arlington, Arlington, Texas, USA

${ }^{9}$ Physics Department, National and Kapodistrian University of Athens, Athens, Greece

${ }^{10}$ Physics Department, National Technical University of Athens, Zografou, Greece

${ }^{11}$ Department of Physics, University of Texas at Austin, Austin, Texas, USA

${ }^{12 a}$ Bahcesehir University, Faculty of Engineering and Natural Sciences, Istanbul, Turkey

${ }^{12 \mathrm{~b}}$ Istanbul Bilgi University, Faculty of Engineering and Natural Sciences, Istanbul, Turkey

${ }^{12 c}$ Department of Physics, Bogazici University, Istanbul, Turkey

${ }^{12 \mathrm{~d}}$ Department of Physics Engineering, Gaziantep University, Gaziantep, Turkey

${ }^{13}$ Institute of Physics, Azerbaijan Academy of Sciences, Baku, Azerbaijan

${ }^{14}$ Institut de Física d'Altes Energies (IFAE), Barcelona Institute of Science and Technology, Barcelona, Spain

${ }^{15 a}$ Institute of High Energy Physics, Chinese Academy of Sciences, Beijing, China

${ }^{15 \mathrm{~b}}$ Physics Department, Tsinghua University, Beijing, China

${ }^{15 c}$ Department of Physics, Nanjing University, Nanjing, China

${ }^{15 \mathrm{~d}}$ University of Chinese Academy of Science (UCAS), Beijing, China

${ }^{16}$ Institute of Physics, University of Belgrade, Belgrade, Serbia

${ }^{17}$ Department for Physics and Technology, University of Bergen, Bergen, Norway

${ }^{18}$ Physics Division, Lawrence Berkeley National Laboratory and University of California, Berkeley, California, USA

${ }^{19}$ Institut für Physik, Humboldt Universität zu Berlin, Berlin, Germany

${ }^{20}$ Albert Einstein Center for Fundamental Physics and Laboratory for High Energy Physics, University of Bern, Bern, Switzerland

${ }^{21}$ School of Physics and Astronomy, University of Birmingham, Birmingham, United Kingdom

${ }^{22 a}$ Facultad de Ciencias y Centro de Investigaciónes, Universidad Antonio Nariño, Bogotá, Colombia

${ }^{22 \mathrm{~b}}$ Departamento de Física, Universidad Nacional de Colombia, Bogotá, Colombia, Colombia

${ }^{23 a}$ INFN Bologna and Universita' di Bologna, Dipartimento di Fisica, Italy

${ }^{23 \mathrm{~b}}$ INFN Sezione di Bologna, Italy

${ }^{24}$ Physikalisches Institut, Universität Bonn, Bonn, Germany

${ }^{25}$ Department of Physics, Boston University, Boston, Massachusetts, USA

${ }^{26}$ Department of Physics, Brandeis University, Waltham, Massachusetts, USA

${ }^{27 a}$ Transilvania University of Brasov, Brasov, Romania

${ }^{27 \mathrm{~b}}$ Horia Hulubei National Institute of Physics and Nuclear Engineering, Bucharest, Romania

${ }^{27 c}$ Department of Physics, Alexandru Ioan Cuza University of Iasi, Iasi, Romania

${ }^{27 \mathrm{~d}}$ National Institute for Research and Development of Isotopic and Molecular Technologies,

Physics Department, Cluj-Napoca, Romania

${ }^{27 \mathrm{e}}$ University Politehnica Bucharest, Bucharest, Romania

${ }^{27 \mathrm{f}}$ West University in Timisoara, Timisoara, Romania

${ }^{28 \mathrm{a}}$ Faculty of Mathematics, Physics and Informatics, Comenius University, Bratislava, Slovak Republic

${ }^{28 b}$ Department of Subnuclear Physics, Institute of Experimental Physics of the Slovak Academy of Sciences, Kosice, Slovak Republic

${ }^{29}$ Physics Department, Brookhaven National Laboratory, Upton, New York, USA

${ }^{30}$ Departamento de Física, Universidad de Buenos Aires, Buenos Aires, Argentina

${ }^{31}$ California State University, California, USA

${ }^{32}$ Cavendish Laboratory, University of Cambridge, Cambridge, United Kingdom

${ }^{33 a}$ Department of Physics, University of Cape Town, Cape Town, South Africa

${ }^{33 b}$ iThemba Labs, Western Cape, South Africa

${ }^{33 c}$ Department of Mechanical Engineering Science, University of Johannesburg, Johannesburg, South Africa

${ }^{33 \mathrm{~d}}$ University of South Africa, Department of Physics, Pretoria, South Africa 
${ }^{33 \mathrm{e}}$ School of Physics, University of the Witwatersrand, Johannesburg, South Africa

${ }^{34}$ Department of Physics, Carleton University, Ottawa, Ontario, Canada

${ }^{35 a}$ Faculté des Sciences Ain Chock, Réseau Universitaire de Physique des Hautes Energies-Université Hassan II, Casablanca, Morocco

${ }^{35 \mathrm{~b}}$ Faculté des Sciences, Université Ibn-Tofail, Kénitra, Morocco

${ }^{35 c}$ Faculté des Sciences Semlalia, Université Cadi Ayyad, LPHEA-Marrakech, Morocco

${ }^{35 \mathrm{~d}}$ Faculté des Sciences, Université Mohamed Premier and LPTPM, Oujda, Morocco

${ }^{35}$ Faculté des sciences, Université Mohammed V, Rabat, Morocco

${ }^{36}$ CERN, Geneva, Switzerland

${ }^{37}$ Enrico Fermi Institute, University of Chicago, Chicago, Illinois, USA

${ }^{38}$ LPC, Université Clermont Auvergne, CNRS/IN2P3, Clermont-Ferrand, France

${ }^{39}$ Nevis Laboratory, Columbia University, Irvington, New York, USA

${ }^{40}$ Niels Bohr Institute, University of Copenhagen, Copenhagen, Denmark

${ }^{41 a}$ Dipartimento di Fisica, Università della Calabria, Rende, Italy

${ }^{41 \mathrm{~b}}$ INFN Gruppo Collegato di Cosenza, Laboratori Nazionali di Frascati, Italy

${ }^{42}$ Physics Department, Southern Methodist University, Dallas, Texas, USA

${ }^{43}$ Physics Department, University of Texas at Dallas, Richardson, Texas, USA

${ }^{44}$ National Centre for Scientific Research “Demokritos”, Agia Paraskevi, Greece

${ }^{45 a}$ Department of Physics, Stockholm University, Sweden

${ }^{45 \mathrm{~b}}$ Oskar Klein Centre, Stockholm, Sweden

${ }^{46}$ Deutsches Elektronen-Synchrotron DESY, Hamburg and Zeuthen, Germany

${ }^{47}$ Lehrstuhl für Experimentelle Physik IV, Technische Universität Dortmund, Dortmund, Germany

${ }^{48}$ Institut für Kern- und Teilchenphysik, Technische Universität Dresden, Dresden, Germany

${ }^{49}$ Department of Physics, Duke University, Durham, North Carolina, USA

${ }^{50}$ SUPA-School of Physics and Astronomy, University of Edinburgh, Edinburgh, United Kingdom

${ }^{51}$ INFN e Laboratori Nazionali di Frascati, Frascati, Italy

${ }^{52}$ Physikalisches Institut, Albert-Ludwigs-Universität Freiburg, Freiburg, Germany

${ }^{53}$ II. Physikalisches Institut, Georg-August-Universität Göttingen, Göttingen, Germany

${ }^{54}$ Département de Physique Nucléaire et Corpusculaire, Université de Genève, Genève, Switzerland

${ }^{55 a}$ Dipartimento di Fisica, Università di Genova, Genova, Italy

${ }^{55 \mathrm{~b}}$ INFN Sezione di Genova, Italy

${ }^{56}$ II. Physikalisches Institut, Justus-Liebig-Universität Giessen, Giessen, Germany

${ }^{57}$ SUPA-School of Physics and Astronomy, University of Glasgow, Glasgow, United Kingdom

${ }^{58}$ LPSC, Université Grenoble Alpes, CNRS/IN2P3, Grenoble INP, Grenoble, France

${ }^{59}$ Laboratory for Particle Physics and Cosmology, Harvard University, Cambridge, Massachusetts, USA

${ }^{60 a}$ Department of Modern Physics and State Key Laboratory of Particle Detection and Electronics, University of Science and Technology of China, Hefei, China

${ }^{60 \mathrm{~b}}$ Institute of Frontier and Interdisciplinary Science and Key Laboratory of Particle Physics and Particle Irradiation (MOE), Shandong University, Qingdao, China

${ }^{60 \mathrm{c}}$ School of Physics and Astronomy, Shanghai Jiao Tong University, KLPPAC-MoE, SKLPPC, Shanghai, China

${ }^{60 \mathrm{~d}}$ Tsung-Dao Lee Institute, Shanghai, China

${ }^{61 \mathrm{a}}$ Kirchhoff-Institut für Physik, Ruprecht-Karls-Universität Heidelberg, Heidelberg, Germany

${ }^{61 \mathrm{~b}}$ Physikalisches Institut, Ruprecht-Karls-Universität Heidelberg, Heidelberg, Germany

${ }^{62}$ Faculty of Applied Information Science, Hiroshima Institute of Technology, Hiroshima, Japan

${ }^{63 a}$ Department of Physics, Chinese University of Hong Kong, Shatin, N.T., Hong Kong, China

${ }^{63 \mathrm{~b}}$ Department of Physics, University of Hong Kong, Hong Kong, China

${ }^{63 \mathrm{c}}$ Department of Physics and Institute for Advanced Study, Hong Kong University of Science and Technology,

Clear Water Bay, Kowloon, Hong Kong, China

${ }^{64}$ Department of Physics, National Tsing Hua University, Hsinchu, Taiwan

${ }^{65}$ IJCLab, Université Paris-Saclay, CNRS/IN2P3, 91405, Orsay, France

${ }^{66}$ Department of Physics, Indiana University, Bloomington, Indiana, USA

${ }^{67 a}$ INFN Gruppo Collegato di Udine, Sezione di Trieste, Udine, Italy

${ }^{67 \mathrm{~b}}$ ICTP, Trieste, Italy

${ }^{67 \mathrm{c}}$ Dipartimento Politecnico di Ingegneria e Architettura, Università di Udine, Udine, Italy

${ }^{68 \mathrm{a}}$ INFN Sezione di Lecce, Italy

${ }^{68 \mathrm{~b}}$ Dipartimento di Matematica e Fisica, Università del Salento, Lecce, Italy

${ }^{69}$ INFN Sezione di Milano, Italy

${ }^{69 \mathrm{~b}}$ Dipartimento di Fisica, Università di Milano, Milano, Italy

${ }^{70 \mathrm{a}}$ INFN Sezione di Napoli, Italy

${ }^{70 \mathrm{~b}}$ Dipartimento di Fisica, Università di Napoli, Napoli, Italy 


\footnotetext{
${ }^{71 \mathrm{a}}$ INFN Sezione di Pavia, Italy

${ }^{71 b}$ Dipartimento di Fisica, Università di Pavia, Pavia, Italy

${ }^{72 a}$ INFN Sezione di Pisa, Italy

${ }^{72 b}$ Dipartimento di Fisica E. Fermi, Università di Pisa, Pisa, Italy

${ }^{73 a}$ INFN Sezione di Roma, Italy

${ }^{73 b}$ Dipartimento di Fisica, Sapienza Università di Roma, Roma, Italy

${ }^{74 a}$ INFN Sezione di Roma Tor Vergata, Italy

${ }^{74 b}$ Dipartimento di Fisica, Università di Roma Tor Vergata, Roma, Italy

${ }^{75 a}$ INFN Sezione di Roma Tre, Italy

${ }^{75 b}$ Dipartimento di Matematica e Fisica, Università Roma Tre, Roma, Italy

${ }^{76 a}$ INFN-TIFPA, Italy

${ }^{76 \mathrm{~b}}$ Università degli Studi di Trento, Trento, Italy

${ }^{77}$ Institut für Astro- und Teilchenphysik, Leopold-Franzens-Universität, Innsbruck, Austria

${ }^{78}$ University of Iowa, Iowa City, Iowa, USA

${ }^{79}$ Department of Physics and Astronomy, Iowa State University, Ames, Iowa, USA

${ }^{80}$ Joint Institute for Nuclear Research, Dubna, Russia

${ }^{81 a}$ Departamento de Engenharia Elétrica, Universidade Federal de Juiz de Fora (UFJF), Juiz de Fora, Brazil

${ }^{81 \mathrm{~b}}$ Universidade Federal do Rio De Janeiro COPPE/EE/IF, Rio de Janeiro, Brazil

${ }^{81 \mathrm{c}}$ Instituto de Física, Universidade de São Paulo, São Paulo, Brazil

${ }^{82}$ KEK, High Energy Accelerator Research Organization, Tsukuba, Japan

${ }^{83}$ Graduate School of Science, Kobe University, Kobe, Japan

${ }^{84 \mathrm{a}}$ AGH University of Science and Technology, Faculty of Physics and Applied Computer Science, Krakow, Poland

${ }^{84 \mathrm{~b}}$ Marian Smoluchowski Institute of Physics, Jagiellonian University, Krakow, Poland

${ }^{85}$ Institute of Nuclear Physics Polish Academy of Sciences, Krakow, Poland

${ }^{86}$ Faculty of Science, Kyoto University, Kyoto, Japan

${ }^{87}$ Kyoto University of Education, Kyoto, Japan

${ }^{88}$ Research Center for Advanced Particle Physics and Department of Physics, Kyushu University, Fukuoka, Japan

${ }^{89}$ Instituto de Física La Plata, Universidad Nacional de La Plata and CONICET, La Plata, Argentina

${ }^{90}$ Physics Department, Lancaster University, Lancaster, United Kingdom

${ }^{91}$ Oliver Lodge Laboratory, University of Liverpool, Liverpool, United Kingdom

${ }^{92}$ Department of Experimental Particle Physics, Jožef Stefan Institute and Department of Physics, University of Ljubljana, Ljubljana, Slovenia

${ }^{93}$ School of Physics and Astronomy, Queen Mary University of London, London, United Kingdom

${ }^{94}$ Department of Physics, Royal Holloway University of London, Egham, United Kingdom

${ }^{95}$ Department of Physics and Astronomy, University College London, London, United Kingdom

${ }^{96}$ Louisiana Tech University, Ruston, Louisiana, USA

${ }^{97}$ Fysiska institutionen, Lunds universitet, Lund, Sweden

${ }^{98}$ Centre de Calcul de l'Institut National de Physique Nucléaire et de Physique des Particules (IN2P3), Villeurbanne, France

${ }^{99}$ Departamento de Física Teorica C-15 and CIAFF, Universidad Autónoma de Madrid, Madrid, Spain

${ }^{100}$ Institut für Physik, Universität Mainz, Mainz, Germany

${ }^{101}$ School of Physics and Astronomy, University of Manchester, Manchester, United Kingdom

${ }^{102}$ CPPM, Aix-Marseille Université, CNRS/IN2P3, Marseille, France

${ }^{103}$ Department of Physics, University of Massachusetts, Amherst, Massachusetts, USA

${ }^{104}$ Department of Physics, McGill University, Montreal, Quebec, Canada

${ }^{105}$ School of Physics, University of Melbourne, Victoria, Australia

${ }^{106}$ Department of Physics, University of Michigan, Ann Arbor, Michigan, USA

${ }^{107}$ Department of Physics and Astronomy, Michigan State University, East Lansing, Michigan, USA

${ }^{108}$ B.I. Stepanov Institute of Physics, National Academy of Sciences of Belarus, Minsk, Belarus

${ }^{109}$ Research Institute for Nuclear Problems of Byelorussian State University, Minsk, Belarus

${ }^{110}$ Group of Particle Physics, University of Montreal, Montreal, Quebec, Canada

${ }^{111}$ P.N. Lebedev Physical Institute of the Russian Academy of Sciences, Moscow, Russia

${ }^{112}$ National Research Nuclear University MEPhI, Moscow, Russia

${ }^{113}$ D.V. Skobeltsyn Institute of Nuclear Physics, M.V. Lomonosov Moscow State University, Moscow, Russia

${ }^{114}$ Fakultät für Physik, Ludwig-Maximilians-Universität München, München, Germany

${ }^{115}$ Max-Planck-Institut für Physik (Werner-Heisenberg-Institut), München, Germany

${ }^{116}$ Nagasaki Institute of Applied Science, Nagasaki, Japan

${ }^{117}$ Graduate School of Science and Kobayashi-Maskawa Institute, Nagoya University, Nagoya, Japan

${ }^{118}$ Department of Physics and Astronomy, University of New Mexico, Albuquerque, New Mexico, USA

${ }^{119}$ Institute for Mathematics, Astrophysics and Particle Physics, Radboud University Nijmegen/Nikhef, Nijmegen, Netherlands

${ }^{120}$ Nikhef National Institute for Subatomic Physics and University of Amsterdam, Amsterdam, Netherlands
} 


\footnotetext{
${ }^{121}$ Department of Physics, Northern Illinois University, DeKalb, Illinois, USA

${ }^{122 a}$ Budker Institute of Nuclear Physics and NSU, SB RAS, Novosibirsk, Russia

${ }^{122 \mathrm{~b}}$ Novosibirsk State University Novosibirsk, Russia

${ }^{123}$ Institute for High Energy Physics of the National Research Centre Kurchatov Institute, Protvino, Russia

${ }^{124}$ Institute for Theoretical and Experimental Physics named by A.I. Alikhanov of National Research Centre "Kurchatov Institute", Moscow, Russia

${ }^{125}$ Department of Physics, New York University, New York, New York, USA

${ }^{126}$ Ochanomizu University, Otsuka, Bunkyo-ku, Tokyo, Japan

${ }^{127}$ Ohio State University, Columbus, Ohio, USA

${ }^{128}$ Homer L. Dodge Department of Physics and Astronomy, University of Oklahoma, Norman, Oklahoma, USA

${ }^{129}$ Department of Physics, Oklahoma State University, Stillwater, Oklahoma, USA

${ }^{130}$ Palacký University, RCPTM, Joint Laboratory of Optics, Olomouc, Czech Republic

${ }^{131}$ Institute for Fundamental Science, University of Oregon, Eugene, Oregon, USA

${ }^{132}$ Graduate School of Science, Osaka University, Osaka, Japan

${ }^{133}$ Department of Physics, University of Oslo, Oslo, Norway

${ }^{134}$ Department of Physics, Oxford University, Oxford, United Kingdom

${ }^{135}$ LPNHE, Sorbonne Université, Université de Paris, CNRS/IN2P3, Paris, France

${ }^{136}$ Department of Physics, University of Pennsylvania, Philadelphia, Pennsylvania, USA

${ }^{137}$ Konstantinov Nuclear Physics Institute of National Research Centre "Kurchatov Institute", PNPI, St. Petersburg, Russia

${ }^{138}$ Department of Physics and Astronomy, University of Pittsburgh, Pittsburgh, Pennsylvania, USA

${ }^{139 a}$ Laboratório de Instrumentação e Física Experimental de Partículas-LIP, Lisboa, Portugal

${ }^{139 b}$ Departamento de Física, Faculdade de Ciências, Universidade de Lisboa, Lisboa, Portugal

${ }^{139 \mathrm{c}}$ Departamento de Física, Universidade de Coimbra, Coimbra, Portugal

${ }^{139 \mathrm{~d}}$ Centro de Física Nuclear da Universidade de Lisboa, Lisboa, Portugal

${ }^{139 \mathrm{e}}$ Departamento de Física, Universidade do Minho, Braga, Portugal

${ }^{139 f}$ Departamento de Física Teórica y del Cosmos, Universidad de Granada, Granada (Spain), Spain

${ }^{139 \mathrm{~g}}$ Dep Física and CEFITEC of Faculdade de Ciências e Tecnologia, Universidade Nova de Lisboa, Caparica, Portugal

${ }^{139 h}$ Instituto Superior Técnico, Universidade de Lisboa, Lisboa, Portugal

${ }^{140}$ Institute of Physics of the Czech Academy of Sciences, Prague, Czech Republic

${ }^{141}$ Czech Technical University in Prague, Prague, Czech Republic

${ }^{142}$ Charles University, Faculty of Mathematics and Physics, Prague, Czech Republic

${ }^{143}$ Particle Physics Department, Rutherford Appleton Laboratory, Didcot, United Kingdom

${ }^{144}$ IRFU, CEA, Université Paris-Saclay, Gif-sur-Yvette, France

${ }^{145}$ Santa Cruz Institute for Particle Physics, University of California Santa Cruz, Santa Cruz, California, USA

${ }^{146 a}$ Departamento de Física, Pontificia Universidad Católica de Chile, Santiago, Chile

${ }^{146 \mathrm{~b}}$ Universidad Andres Bello, Department of Physics, Santiago, Chile

${ }^{146 c}$ Instituto de Alta Investigación, Universidad de Tarapacá, Chile

${ }^{146 \mathrm{~d} D e p a r t a m e n t o ~ d e ~ F i ́ s i c a, ~ U n i v e r s i d a d ~ T e ́ c n i c a ~ F e d e r i c o ~ S a n t a ~ M a r i ́ a, ~ V a l p a r a i ́ s o, ~ C h i l e ~}$

${ }^{147}$ Universidade Federal de São João del Rei (UFSJ), São João del Rei, Brazil

${ }^{148}$ Department of Physics, University of Washington, Seattle, Washington, USA

${ }^{149}$ Department of Physics and Astronomy, University of Sheffield, Sheffield, United Kingdom

${ }^{150}$ Department of Physics, Shinshu University, Nagano, Japan

${ }^{151}$ Department Physik, Universität Siegen, Siegen, Germany

${ }^{152}$ Department of Physics, Simon Fraser University, Burnaby, British Columbia, Canada

${ }^{153}$ SLAC National Accelerator Laboratory, Stanford, California, USA

${ }^{154}$ Physics Department, Royal Institute of Technology, Stockholm, Sweden

${ }^{155}$ Departments of Physics and Astronomy, Stony Brook University, Stony Brook, New York, USA

${ }^{156}$ Department of Physics and Astronomy, University of Sussex, Brighton, United Kingdom

${ }^{157}$ School of Physics, University of Sydney, Sydney, Australia

${ }^{158}$ Institute of Physics, Academia Sinica, Taipei, Taiwan

${ }^{159 a}$ E. Andronikashvili Institute of Physics, Iv. Javakhishvili Tbilisi State University, Tbilisi, Georgia

${ }^{159 \mathrm{~b}}$ High Energy Physics Institute, Tbilisi State University, Tbilisi, Georgia

${ }^{160}$ Department of Physics, Technion, Israel Institute of Technology, Haifa, Israel

${ }^{161}$ Raymond and Beverly Sackler School of Physics and Astronomy, Tel Aviv University, Tel Aviv, Israel

${ }^{162}$ Department of Physics, Aristotle University of Thessaloniki, Thessaloniki, Greece

${ }^{163}$ International Center for Elementary Particle Physics and Department of Physics, University of Tokyo, Tokyo, Japan

${ }^{164}$ Graduate School of Science and Technology, Tokyo Metropolitan University, Tokyo, Japan

${ }^{165}$ Department of Physics, Tokyo Institute of Technology, Tokyo, Japan

${ }^{166}$ Tomsk State University, Tomsk, Russia

${ }^{167}$ Department of Physics, University of Toronto, Toronto, Ontario, Canada
} 


\section{${ }^{168 a}$ TRIUMF, Vancouver, British Columbia, Canada \\ ${ }^{168 b}$ Department of Physics and Astronomy, York University, Toronto, Ontario, Canada \\ ${ }^{169}$ Division of Physics and Tomonaga Center for the History of the Universe, Faculty of Pure and Applied Sciences, University of Tsukuba, Tsukuba, Japan \\ ${ }^{170}$ Department of Physics and Astronomy, Tufts University, Medford, Massachusetts, USA \\ ${ }^{171}$ Department of Physics and Astronomy, University of California Irvine, Irvine, California, USA \\ ${ }^{172}$ Department of Physics and Astronomy, University of Uppsala, Uppsala, Sweden \\ ${ }^{173}$ Department of Physics, University of Illinois, Urbana, Illinois, USA \\ ${ }^{174}$ Instituto de Física Corpuscular (IFIC), Centro Mixto Universidad de Valencia-CSIC, Valencia, Spain \\ ${ }^{175}$ Department of Physics, University of British Columbia, Vancouver, British Columbia, Canada \\ ${ }^{176}$ Department of Physics and Astronomy, University of Victoria, Victoria, British Columbia, Canada \\ ${ }^{177}$ Fakultät für Physik und Astronomie, Julius-Maximilians-Universität Würzburg, Würzburg, Germany \\ ${ }^{178}$ Department of Physics, University of Warwick, Coventry, United Kingdom \\ ${ }^{179}$ Waseda University, Tokyo, Japan \\ ${ }^{180}$ Department of Particle Physics and Astrophysics, Weizmann Institute of Science, Rehovot, Israel \\ ${ }^{181}$ Department of Physics, University of Wisconsin, Madison, Wisconsin, USA \\ ${ }^{182}$ Fakultät für Mathematik und Naturwissenschaften, Fachgruppe Physik, Bergische Universität Wuppertal, Wuppertal, Germany \\ ${ }^{183}$ Department of Physics, Yale University, New Haven, Connecticut, USA}

${ }^{\mathrm{a}}$ Deceased.

${ }^{\mathrm{b}}$ Also at Department of Physics, King's College London, London, United Kingdom.

${ }^{c}$ Also at Instituto de Fisica Teorica, IFT-UAM/CSIC, Madrid, Spain.

${ }^{\mathrm{d}}$ Also at TRIUMF, Vancouver, British Columbia, Canada.

eAlso at Department of Physics and Astronomy, University of Louisville, Louisville, Kentucky, USA.

${ }^{\mathrm{f}}$ Also at Physics Department, An-Najah National University, Nablus, Palestine.

${ }^{\mathrm{g}}$ Also at Department of Physics, University of Fribourg, Fribourg, Switzerland.

${ }^{\mathrm{h}}$ Also at Departament de Fisica de la Universitat Autonoma de Barcelona, Barcelona, Spain.

${ }^{\mathrm{i}}$ Also at Moscow Institute of Physics and Technology State University, Dolgoprudny, Russia.

${ }^{\mathrm{j}}$ Also at Department of Physics, Ben Gurion University of the Negev, Beer Sheva, Israel.

${ }^{k}$ Also at Universita di Napoli Parthenope, Napoli, Italy.

${ }^{1}$ Also at Institute of Particle Physics (IPP), Canada.

${ }^{\mathrm{m}}$ Also at Department of Physics, St. Petersburg State Polytechnical University, St. Petersburg, Russia.

${ }^{\mathrm{n}}$ Also at Borough of Manhattan Community College, City University of New York, New York, New York, USA.

${ }^{\circ}$ Also at Department of Physics, California State University, Fresno, USA.

${ }^{\mathrm{p}}$ Also at Department of Financial and Management Engineering, University of the Aegean, Chios, Greece.

${ }^{\mathrm{q}}$ Also at Centro Studi e Ricerche Enrico Fermi, Italy.

${ }^{\mathrm{r}}$ Also at Department of Physics, California State University, East Bay, USA.

${ }^{\mathrm{s}}$ Also at Institucio Catalana de Recerca i Estudis Avancats, ICREA, Barcelona, Spain.

${ }^{\mathrm{t}}$ Also at Graduate School of Science, Osaka University, Osaka, Japan.

${ }^{\mathrm{u}}$ Also at Physikalisches Institut, Albert-Ludwigs-Universität Freiburg, Freiburg, Germany.

${ }^{v}$ Also at University of Chinese Academy of Sciences (UCAS), Beijing, China.

${ }^{\mathrm{w}}$ Also at Institute of Physics, Azerbaijan Academy of Sciences, Baku, Azerbaijan.

${ }^{\mathrm{x}}$ Also at CERN, Geneva, Switzerland.

${ }^{y}$ Also at Joint Institute for Nuclear Research, Dubna, Russia.

${ }^{\mathrm{z}}$ Also at Hellenic Open University, Patras, Greece.

${ }^{\text {aa }}$ Also at Center for High Energy Physics, Peking University, China.

${ }^{\mathrm{bb}}$ Also at The City College of New York, New York, New York, USA.

${ }^{c c}$ Also at Dipartimento di Matematica, Informatica e Fisica, Università di Udine, Udine, Italy.

${ }^{\mathrm{dd}}$ Also at Department of Physics, California State University, Sacramento, USA.

${ }^{\mathrm{ee}}$ Also at Département de Physique Nucléaire et Corpusculaire, Université de Genève, Genève, Switzerland.

${ }^{\mathrm{ff}}$ Also at Institute for Nuclear Research and Nuclear Energy (INRNE) of the Bulgarian Academy of Sciences, Sofia, Bulgaria.

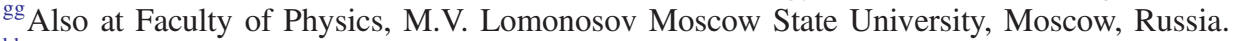

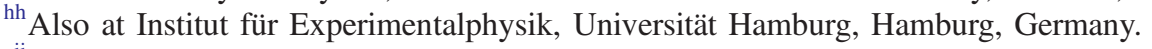

${ }^{\text {ii }}$ Also at CPPM, Aix-Marseille Université, CNRS/IN2P3, Marseille, France.

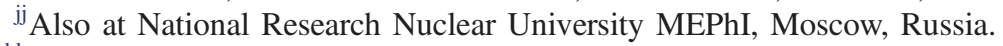

${ }^{\mathrm{kk}}$ Also at Institute for Particle and Nuclear Physics, Wigner Research Centre for Physics, Budapest, Hungary.

${ }^{11}$ Also at Giresun University, Faculty of Engineering, Giresun, Turkey.

${ }^{\mathrm{mm}}$ Also at Department of Physics and Astronomy, Michigan State University, East Lansing, Michigan, USA. 